\author{
Universidade de Brasília \\ Instituto de Ciências Exatas \\ Departamento de Matemática
}

\title{
Existência de soluções para problemas quasilineares com dados em espaços de medida
}

\author{
por \\ Welber Faustino da Silva \\ Orientador: Luís Henrique de Miranda \\ Brasília \\ 2016
}


Ficha catalográfica elaborada automaticamente, com os dados fornecidos pelo(a) autor(a)

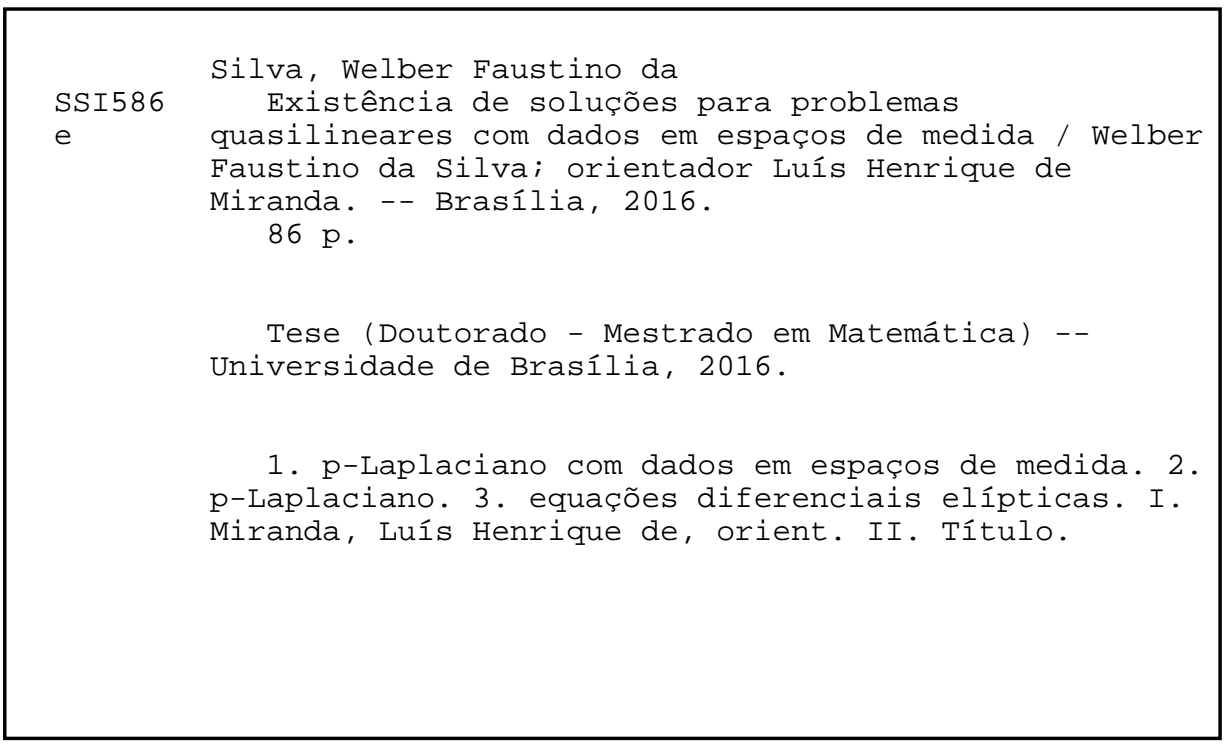




\title{
Existência de soluções para problemas quasilineares com dados em espaços de medida
}

\author{
por \\ Welber Faustino da Silva * \\ Dissertação apresentada ao Departamento de Matemática da Universidade \\ de Brasília, como parte dos requisitos para obtenção do grau de
}

\section{MESTRE EM MATEMÁTICA}

Brasília, 10 de março de 2016.

Comissão Examinadora:

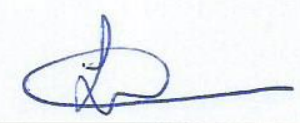

Prof. Dr. Luis Henrique de Miranda - MAT/UnB (Orientador)
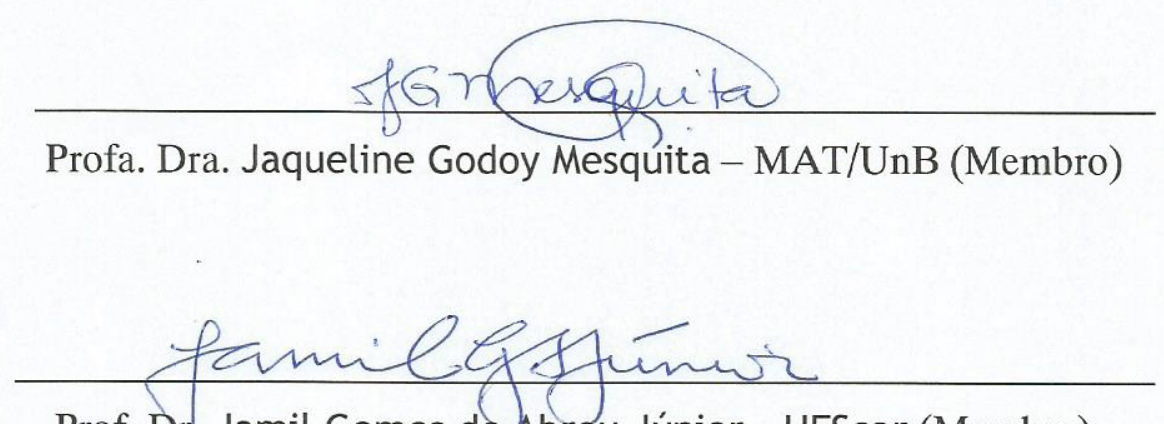

Prof. Dr. Jamil Gomes de Abreu Júnior - UFScar (Membro)

* O autor foi bolsista do CNPq durante a elaboração desta dissertação. 
À Rute Silva, em memória. 
Dedico esse trabalho, ̀̀ Dona Raquel Silva, Seu José Venceslau da Silva, e a minha amada, Luciolle Ferreira de Araújo, 


\section{Agradecimentos}

À Deus pelo dom da vida. Este dom que muitos hoje desprezam, mas que está presente em cada ser vivente, independente de que acredite ou não.

Ao professor e orientador Luís Henrique de Miranda, por toda sua dedicação e paciência para minhas intermináveis dúvidas. O agradeço por se dispor a me ajudar principalmente nos momentos mais difíceis deste presente trabalho.

Aos professores Jamil Gomes de Abreu Júnior e Jaqueline Godoy Mesquita por terem aceitado participarem da banca examinadora.

À minha noiva e amada Luciolle Ferreira da Araújo, por seu apoio e compreensão. Obrigado por estar ao meu lado me incentivando e me fortalecendo com suas palavras e carinho.

À minha mãe Raquel Silva, grande guerreira, trabalhadora, que não mediu esforços para eu estar aqui. Sempre me incentivou a estudar e me corrigia nos momentos certos.

Ao meu padrasto José Venceslau da Silva, por seu amor como de um verdadeiro pai.

Aos meus irmãos Karen Priscila Silva, David Faustino da Silva e Wesley Faustino da Silva, pelo incentivo a continuar estudando.

Aos amigos que fiz no Departamento de Matemática da UnB durante esses dois anos. Principalmente aqueles que estiveram ao meu lado nos momentos mais difíceis.

À todos os professores do Departamento de Matemática da UnB que contribuíram para minha formação.

Aos funcionários do Departamento de Matemática da UnB, em especial, aos porteiros da sala de estudo do mestrado. Por vezes, tiveram que me "aturar" nas minhas madrugadas de estudo.

Ao CNPq pelo apoio financeiro durante esses dois anos de mestrado. 


\section{Resumo}

Neste trabalho, estudaremos a existência de soluções fracas para os problemas:

$$
\left\{\begin{array}{rll}
-\Delta_{p} u= & \text { em } \Omega \\
u= & 0 & \text { em } \partial \Omega
\end{array}\right.
$$

e

$$
\left\{\begin{array}{rlll}
-\Delta_{p} u+g(x, u) & = & f & \text { em } \Omega \\
u & = & 0 & \text { em } \partial \Omega
\end{array}\right.
$$

onde $\Omega \subset \mathbb{R}^{N}(N \geq 2)$ é um aberto, limitado, conexo e $f \in M(\Omega)$. A função $g$ satisfaz, $a$ priori, as seguintes hipóteses:

- $g(x, s)$ é mensurável em $x \in \Omega, \forall s \in \mathbb{R}$ e contínua em $s \in \mathbb{R}$, para q.t.p. $x \in \Omega$;

- $g(x, s) s \geq 0 \forall s \in \mathbb{R}$, q.t.p. em $x \in \Omega$.

Palavras-chave: p-Laplaciano com dados em espaços de medida, p-Laplaciano, equações diferenciais elípticas. 


\section{Abstract}

In this work, we will study the existence of weak solutions for the problems:

$$
\left\{\begin{aligned}
-\Delta_{p} u=f & \text { in } \Omega \\
u=0 & \text { on } \partial \Omega
\end{aligned}\right.
$$

and

$$
\left\{\begin{array}{rll}
-\Delta_{p} u+g(x, u) & =f & \text { in } \Omega \\
u & =0 & \text { on } \partial \Omega
\end{array}\right.
$$

where $\Omega \subset \mathbb{R}^{N}(N \geq 2)$ is open, bounded, connected and $f \in M(\Omega)$. The function $g$ satisfies, a priori, the following hypotheses:

- $g(x, s)$ is measurable in $x \in \Omega$, forall $s \in \mathbb{R}$ and continuous in $s \in \mathbb{R}$, a.e. $x \in \Omega$;

- $g(x, s) s \geq 0 \forall s \in \mathbb{R}$, a.e. in $x \in \Omega$.

Keywords: p-Laplacian involving measure data, p-Laplacian, elliptic differential equations. 


\section{Notações}

Neste trabalho, usaremos as seguintes notações:

$$
\begin{aligned}
& p^{\prime}=\frac{p}{p-1}, \\
& p^{*}=\frac{N p}{N-p}, \\
& u_{n} \rightarrow u, \\
& u_{n} \rightarrow u, \\
& \hookrightarrow, \\
& \hookrightarrow \hookrightarrow, \\
& \nabla u=\left(\frac{\partial u}{\partial x_{1}}, \frac{\partial u}{\partial x_{2}}, \cdots, \frac{\partial u}{\partial x_{N}}\right) \\
& -\Delta_{p} u=-\operatorname{div}\left(|\nabla u|^{p-2} \nabla u\right), \\
& \text { suppf, } \\
& \mathbb{R}^{+} \\
& M(\Omega) \\
& C(\Omega) \\
& C_{c}(\Omega), \\
& \text { conjugado de } p \text {. } \\
& \text { expoente crítico de Sobolev. } \\
& \text { convergência forte (em norma). } \\
& \text { convergência fraca. } \\
& \text { imersão contínua de um espaço em outro. } \\
& \text { imersão compacta de um espaço em outro. } \\
& \text { gradiente de } u \text {. } \\
& p \text {-Laplaciano de } u \text {. } \\
& \text { suporte da função } f \text {. } \\
& \text { conjunto dos números reais não negativos. } \\
& \text { conjunto das medidas de Radon finitas sobre } \Omega \text {. } \\
& \text { conjunto das funções contínuas. } \\
& f \in C(\Omega) \text { tal que suppf é compacto. }
\end{aligned}
$$


$D(\Omega)$,

$D^{\prime}(\Omega)$,

$\|u\|_{L^{p}}=\left[\int_{\Omega}|u|^{p}\right]^{1 / p}$

$L^{p}(\Omega)=\left\{u: \Omega \rightarrow \mathbb{R}\right.$ mensurável; $\left.\|u\|_{L^{p}}<\infty\right\}$.

$L_{\text {loc }}^{p}(\Omega)=\left\{u: \Omega \rightarrow \mathbb{R}\right.$ mensurável; $\left.u\right|_{K} \in L^{p}(\Omega), \forall K \subset \subset \Omega$ compacto $\}$.

$W^{1, p}(\Omega)=\left\{u: \Omega \longrightarrow \mathbb{R}: u, \frac{\partial u}{\partial x_{i}} \in L^{p}(\Omega), i=1, \ldots, N\right\}$.

$W_{0}^{1, p}(\Omega)={\overline{C_{c}^{\infty}(\Omega)}}^{\|\|_{W^{1, p}}}$.

$\|u\|_{L^{p}}=\left[\int_{\Omega}|u|^{p} d \mu\right]^{1 / p}$,

$L^{p}(X, \mu)$, espaço das funções teste.

espaço das distribuições em $\Omega$.

em relação a medida de Lebesgue. em relação a uma medida positiva.

em relação a uma medida positiva. 


\section{Sumário}

$\begin{array}{lc}\text { Introdução } & 1\end{array}$

1 Preliminares $\quad 4$

1.1 Medidas de Radon . . . . . . . . . . . . . . . . . . . . . . . . . . 4

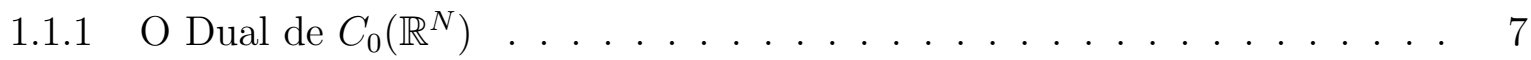

1.1.2 Densidade Vaga de $L^{1}\left(\mathbb{R}^{N}\right)$ em $M\left(\mathbb{R}^{N}\right) \ldots \ldots \ldots \ldots$. . . . . . . 13

1.1 .3 Distribuições . . . . . . . . . . . . . . . . . . . . 18

1.2 Espaços de Sobolev . . . . . . . . . . . . . . . . . . . . . 20

1.3 Grau de Brouwer . . . . . . . . . . . . . . . . . . . 22

1.4 Teoremas de Convergência . . . . . . . . . . . . . . . . . . . 23

2 Existência de Solução para dados em $W^{-1, p^{\prime}}(\Omega) \quad 29$

2.1 Operadores Monótonos . . . . . . . . . . . . . . . . . . . . 29

2.2 Existência de Solução para o p-Laplaciano . . . . . . . . . . . . . . . . 35

3 Existência de Solução para dados em $M(\Omega) \quad 45$

3.1 Existência de Solução para o p-Laplaciano . . . . . . . . . . . . . . . . . . 45

3.2 Continuidade para Regularidades Intermediárias . . . . . . . . . . . . . 58

3.3 Efeitos das Perturbações Semilineares . . . . . . . . . . . . . . . . 65

$\begin{array}{ll}\text { Referências Bibliográficas } & 75\end{array}$ 


\section{Introdução}

Neste trabalho, estudaremos existência de soluções fracas para duas classes de problemas envolvendo o operador $p$-Laplaciano. O primeiro problema a tratarmos será:

$$
(P) \quad\left\{\begin{array}{rll}
-\Delta_{p} u= & \text { em } \Omega \\
u= & 0 \quad \text { sobre } \partial \Omega
\end{array}\right.
$$

onde $\Omega \subset \mathbb{R}^{N}(N \geq 2)$ é um aberto, limitado, conexo e $f \in M(\Omega)$. O conjunto $M(\Omega)$ denota o conjunto das medidas de Radon finitas sobre $M(\Omega)$.

No trabalho de Boccardo \& Gallouet [2], foi investigada a existência de soluções fracas para o problema $(P)$ para uma classe de operadores mais gerais. Eles consideraram o seguinte operador:

$$
A u=-\operatorname{div}(a(x, \nabla u))
$$

onde $A$ é um operador monótono e a satisfazendo certas hipóteses. Observamos que o operador $p$-Laplaciano é um caso particular de $A$.

Já o segundo problema é o caso semilinear de (P), ou seja,

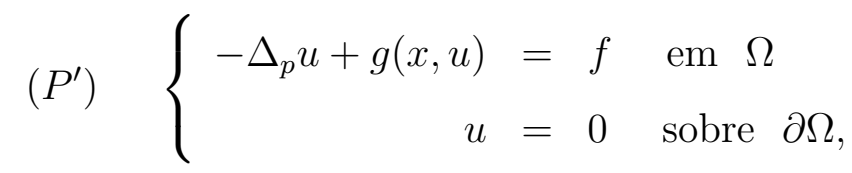

onde a função $g$ satisfaz as seguintes hipóteses:

(1) $g(x, s)$ é mensurável em $x \in \Omega, \forall s \in \mathbb{R}$ e contínua em $s \in \mathbb{R}$, para q.t.p. $x \in \Omega$;

(2) $g(x, s) s \geq 0, \forall s \in \mathbb{R}$, para q.t.p. $x \in \Omega$.

Para o problema $\left(P^{\prime}\right)$, queremos encontrar soluções fracas quando $f \in L^{1}(\Omega)$ ou $f \in M(\Omega)$.

Como em $(P)$, Boccardo \& Gallouet também estudaram o problema $\left(P^{\prime}\right)$, considerando o operador $A=-\operatorname{div}(a(x, \nabla u))$. Foi observado que para obtermos existência de soluções fracas quando $f \in L^{1}(\Omega)$, precisamos adicionar a seguinte hipótese sobre $g$ :

(3) $\sup \{|g(x, s)|,|s| \leq t\} \in L_{l o c}^{1}(\Omega), \forall t \in \mathbb{R}^{+}$. 
Quando $f \in M(\Omega)$, é necessária uma hipótese mais forte, a saber:

$$
\text { existem } b_{1}, b_{2}, \delta \text { com } b_{1} \in L_{l o c}^{1}(\Omega), b_{2} \in L_{l o c}^{\infty}(\Omega),
$$

$$
\delta<N(p-1) /(N-p) \text {, tal que }|g(x, s)| \leq b_{1}(x)+b_{2}(x)|s|^{\delta}
$$

q.t.p em $x$, para todo número real $s$.

As dificuldades de se encontrar soluções fracas para os problema $(P)$ e $\left(P^{\prime}\right)$ surgem já quando consideramos $f \in L^{1}(\Omega)$, pois o espaço $L^{1}(\Omega)$ não é reflexivo e, assim, argumentos de convergência na topologia fraca de $L^{1}(\Omega)$ geralmente falham. Observamos também que, em geral, para o caso $f \in L^{1}(\Omega)$, a obtenção de estimativas a priori é mais complicada.

Por exemplo, consideremos $1<p<N$ e seja $u$ uma solução distribucional de $(P)$, isto é,

$$
\int_{\Omega}|\nabla u|^{p-2} \nabla u \cdot \nabla \varphi=\int_{\Omega} f \varphi, \quad \forall \varphi \in C_{c}^{\infty}(\Omega) .
$$

Se $f \in L^{m}(\Omega)$, com $m \geq p^{\prime}$, temos $L^{m}(\Omega) \hookrightarrow L^{p^{\prime}}(\Omega) \hookrightarrow W^{-1, p^{\prime}}(\Omega)$ e, assim, pode-se mostrar que $u \in W_{0}^{1, p}(\Omega)$ (veja Teorema 2.2.5, pág. 38), e a obtenção de estimativas a priori não é um problema, já que $|\nabla u|^{p-1} \in L^{p^{\prime}}(\Omega)$. Assim, a classe das funções teste pode ser estendida para $W_{0}^{1, p}(\Omega)$. Logo, $u$ é uma função teste válida e tomando-se $u=\varphi$ em (5), pela desigualdade de Hölder, temos:

$$
\int_{\Omega}|\nabla u|^{p} \leq\|u\|_{L^{p}}\|f\|_{L^{p^{\prime}}}
$$

Usando a desigualdade de Poincaré e com algumas manipulações simples, obtemos:

$$
\|u\|_{W_{0}^{1, p}} \leq C\|f\|_{L^{p^{\prime}}}^{p^{\prime} / p}
$$

No caso em que $f \in L^{1}(\Omega)$, pode-se mostrar que $u \in W_{0}^{1, q}(\Omega)$, onde

$$
1 \leq q<\frac{N}{N-1}(p-1)
$$

Assim,

$$
|\nabla u|^{p-1} \in L^{r}(\Omega) \quad \operatorname{com} r \in\left[1, \frac{N}{N-1}\right) .
$$

Logo, a classe das funções teste pode ser, na melhor das hipóteses, estendida ao espaço

$$
L^{r^{\prime}}(\Omega), \quad \text { onde } r^{\prime}>N
$$

Porém, $N>\frac{N}{N-1}(p-1)$ e então $u$ não pode ser tomado como função teste, o que dificulta substancialmente até mesmo a obtenção de estimativas a priori mais básicas. 
Seguindo Boccardo \& Gallouet, consideramos como função teste uma função da forma $\psi(u)$, onde $\psi: \mathbb{R} \longrightarrow \mathbb{R}$ será uma função localmente Lipschitz escolhida com certo cuidado. Neste caso, é possível tomar $\varphi=\psi(u)$ como função teste e o desafio é recuperar alguma estimativa a priori, pois será necessário controlar o gradiente $\nabla \psi(u)$.

Para estudarmos os problemas $(P)$ e $\left(P^{\prime}\right)$, usaremos como ferramenta básica um resultado do trabalho de Leray-Lions [13]. Ressaltamos que o método usado em [13] foi influenciado por trabalhos como o de Browder [5], [6]; Minty [15], [16], [17], e também Visik [19] e [20]. A técnica usada nestes trabalhos, em geral baseia-se na combinação de problemas aproximados, como o "Método de Galerkin", e argumentos que exploram a monotonicidade do operador diferencial em questão.

Observamos que nos problemas $(P)$ e $\left(P^{\prime}\right)$ consideraremos $p \in(2-1 / N, N]$. A limitação inferior para $p$ é imposta à medida que estamos preocupados com o limite inferior para que $u \in W_{0}^{1,1}(\Omega)$. Notamos também que, quando $p>N$, sabe-se que ambos os problemas têm uma única solução fraca em $W_{0}^{1, p}(\Omega)$ quando incluímos $M(\Omega)$ em $W^{-1, p^{\prime}}(\Omega)$ (ver [13]).

O presente texto está organizado da seguinte maneira. No Capítulo 1, abordamos os assuntos preliminares. Este capítulo foi dividido em 2 seções. Na primeira seção, fazemos uma introdução às Medidas de Radon. Estamos interessados em obter suas principais propriedades e mostraremos que o dual de $C_{0}\left(\mathbb{R}^{N}\right)$ pode ser "representado" como o espaço das medidas de Radon com sinal $M\left(\mathbb{R}^{N}\right)$, afim, de definirmos a topologia fraca sobre $M\left(\mathbb{R}^{N}\right)$. Posteriormente, discutiremos como as medidas de Radon se relacionam com funções em $L^{1}$ ou distribuições. A terceira seção é dedicada a apresentar os teoremas de convergência mais importantes da teoria da medida e integração.

No Capítulo 2, investigaremos versões preliminares de $(P)$ e $\left(P^{\prime}\right)$, quando o termo forçante pertence a espaços mais regulares do que os das medidas de Radon. Este capítulo é inspirado nos trabalhos de Ciarlet [7] e de Leray-Lions [13].

O Capítulo 3 é o mais importante desta dissertação. Nele, combinaremos as técnicas discorridas ao longo do texto com a obtenção de estimativas a priori delicadas para mostrar a existência de soluções fracas para $(P)$ e $\left(P^{\prime}\right)$. Este capítulo é inspirado no trabalho de Boccardo \& Gallouet [2]. 


\section{Capítulo 1}

\section{Preliminares}

Este capítulo tem como objetivo dar o suporte matemático necessário para os Capítulos 2 e 3. Na Seção 1.1 faremos uma introdução às Medidas de Radon em $\mathbb{R}^{N}$ e apresentaremos os principais resultados que serão utilizados adiante. O primeiro objetivo é mostrar que o espaço das medidas de Radon $M\left(\mathbb{R}^{N}\right)$ é isometricamente isomorfo ao espaço dual do conjunto das funções contínuas que se anulam no infinito, e assim definirmos a noção de topologia fraca em $M\left(\mathbb{R}^{N}\right)$. Posteriormente, vamos mostrar que $L^{1}\left(\mathbb{R}^{N}\right)$ é denso em $M\left(\mathbb{R}^{N}\right)$ nesta topologia.

Na Seção 1.2, apresentamos os principais teoremas de convergência quando consideramos um espaço de medida positiva.

Para o que segue, admitimos que o leitor tenha conhecimentos básicos sobre Teoria da Medida e Integração, Espaços $L^{p}$ e os Espaços de Sobolev.

\subsection{Medidas de Radon}

Nesta seção, definiremos as medidas de Radon sobre o espaço $\mathbb{R}^{N}$. Estudaremos suas principais propriedades, sua representação com o dual do conjunto das funções contínuas que se anulam no infinito, a densidade de $L^{1}$ no espaço das medidas de Radon sobre $\mathbb{R}^{N}$ relativamente à topologia fraca desse espaço e, ao final, trataremos da sua relação com a convergência em distribuição. Observamos que a maior parte desta seção é baseada em [9].

Seja $f: \mathbb{R}^{N} \rightarrow \mathbb{R}$. Definimos o suporte de $f$ como sendo o fecho do conjunto onde $f$ não se anula, ou seja,

$$
\operatorname{supp}(f)=\overline{\{x: f(x) \neq 0\}}
$$


Além disso, definimos os seguintes conjuntos:

$$
C\left(\mathbb{R}^{N}\right)=\left\{f: \mathbb{R}^{N} \rightarrow \mathbb{R} ; f \text { é contínua }\right\}
$$

e

$$
C_{c}\left(\mathbb{R}^{N}\right)=\left\{f \in C\left(\mathbb{R}^{N}\right): \operatorname{supp}(f) \text { é compacto }\right\}
$$

Em $C_{c}\left(\mathbb{R}^{N}\right)$, definimos a seguinte norma:

$$
\|f\|_{u}=\sup \left\{|f(x)|: x \in \mathbb{R}^{N}\right\}
$$

e assim, $C_{c}\left(\mathbb{R}^{N}\right)$ é um espaço vetorial normado.

Dada $f: \mathbb{R}^{N} \longrightarrow \mathbb{R}^{+}$, com $f$ contínua, temos que:

$$
\psi(f)=\int_{\mathbb{R}^{N}} f d \mu \geq 0
$$

onde $\mu$ é a medida de Lebesgue sobre $\mathbb{R}^{N}$. Isso motiva a seguinte definição.

Definição 1.1.1. Dizemos que um funcional linear I definido em $C_{c}\left(\mathbb{R}^{N}\right)$ é positivo se

$$
I(f) \geq 0 \quad \text { para toda } f \geq 0 .
$$

A definição de funcional linear positivo nos dá uma noção de continuidade. Esse fato é tratado na seguinte proposição.

Proposição 1.1.2. Se I é um funcional linear positivo em $C_{c}\left(\mathbb{R}^{N}\right)$, então para cada compacto $K \subset \mathbb{R}^{N}$ existe uma constante $C_{K}$ tal que:

$$
|I(f)| \leq C_{K}\|f\|_{u} \text {, para toda } f \in C_{c}\left(\mathbb{R}^{N}\right) \text { tal que supp }(f) \subset K
$$

Demonstração. Ver [9], pág. 212, Proposição 7.1.

Lembramos que uma medida de Borel sobre $\mathbb{R}^{N}$ é uma medida definida na $\sigma$-álgebra $\mathcal{B}_{\mathbb{R}^{N}}$, onde $\mathcal{B}_{\mathbb{R}^{N}}$ denota o conjuntos dos borelianos de $\mathbb{R}^{N}$.

Dada $\mu$ uma medida de Borel sobre $\mathbb{R}^{N}$, se $\mu(K)<\infty$ para todo compacto $K \subset \mathbb{R}^{N}$, então:

$$
C_{c}\left(\mathbb{R}^{N}\right) \subset L^{1}\left(\mathbb{R}^{N}\right)
$$

De fato, seja $f \in C_{c}\left(\mathbb{R}^{N}\right)$, então

$$
\int_{\mathbb{R}^{N}} f d \mu=\int_{K} f d \mu \leq\|f\|_{u} \mu(K)<\infty .
$$


Logo, $f \in L^{1}\left(\mathbb{R}^{N}\right)$. Além disso, note que a aplicação

$$
f \longmapsto \int_{\mathbb{R}^{N}} f d \mu
$$

é um funcional linear positivo agindo sobre $C_{c}\left(\mathbb{R}^{N}\right)$.

Mostraremos adiante, que todo funcional positivo definido em $C_{c}\left(\mathbb{R}^{N}\right)$ pode ser representado dessa forma. Além disso, sob certas hipóteses de regularidade na medida $\mu$ podemos garantir a unicidade da medida.

A seguir, iremos definir a medida $\mu$ com a qual poderemos representar de forma única todos os funcionais lineares positivos definidos em $C_{c}\left(\mathbb{R}^{N}\right)$.

Definição 1.1.3. Uma medida de Radon $\mu$ sobre $\mathbb{R}^{N}$ é uma medida positiva de Borel que satisfaz:

(i) $\mu(K)<\infty$, para todo $K \subset \mathbb{R}^{N}$, compacto;

(ii) Dado $V \subset \mathbb{R}^{N}$ aberto, então

$$
\mu(V)=\sup \{\mu(K): K \subset V \text { e } K \text { compacto }\}
$$

(iii) Dado $A \in \mathcal{B}_{\mathbb{R}^{N}}$, então

$$
\mu(A)=\inf \{\mu(V): A \subset V \text { e } V \text { aberto }\}
$$

Considerando a $\sigma$-álgebra gerada pelos boreleanos de $\mathbb{R}^{N}$ no aberto $\Omega \subset \mathbb{R}^{N}$, definimos de maneira análoga o conceito de medida de Radon sobre $\Omega$.

Dois exemplos básicos de medidas de Radon são dados abaixo.

Exemplo 1.1.4. A medida de Lebesgue é uma medida de Radon.

Exemplo 1.1.5. A medida definida por:

$$
\delta_{y}(E)= \begin{cases}1, & \text { se } y \in E \\ 0, & \text { caso contrário }\end{cases}
$$

é outro exemplo de medida de Radon, chamada de Delta Dirac

O próximo resultado nos diz que todo funcional linear positivo em $C_{c}\left(\mathbb{R}^{N}\right)$ pode ser representado como uma integral com respeito a uma única medida de Radon. 
Teorema 1.1.6. (Representação de Riez) Seja $I: C_{c}\left(\mathbb{R}^{N}\right) \longrightarrow \mathbb{R}$ um funcional linear positivo. Então existe uma única medida de Radon $\mu$ sobre $\mathbb{R}^{N}$ tal que:

$$
I(f)=\int_{\mathbb{R}^{N}} f d \mu, \quad \forall f \in C_{c}\left(\mathbb{R}^{N}\right) .
$$

Além disso, temos que $\mu$ satisfaz:

$$
\mu(O)=\sup \left\{I(f): f \in C_{c}\left(\mathbb{R}^{N}\right), 0 \leq f \leq 1 \text { e supp }(f) \subset O\right\} \text { para todo aberto } O \subset \mathbb{R}^{N}
$$

e

$$
\mu(K)=\sup \left\{I(f): f \in C_{c}\left(\mathbb{R}^{N}\right) \text { e } f \geq \mathcal{X}_{K}\right\} \text { para todo compacto } K \subset \mathbb{R}^{N}
$$

Demonstração. Ver [9], pág. 212, Teorema 7.2.

O teorema a seguir será essencial para o resultado principal da próxima subseção. Intuitivamente ele nos diz que toda função mensurável cujo o conjuntos dos pontos $\left\{x \in \mathbb{R}^{N}: f(x) \neq 0\right\}$ tem medida finita é "quase contínua" para todo conjunto compacto.

Teorema 1.1.7 (Lusin). Suponha que $\mu$ seja uma medida de Radon sobre $\mathbb{R}^{N}$ e seja $f: \mathbb{R}^{N} \rightarrow \mathbb{R}$ uma função mensurável tal que $\mu\left(\left\{x \in \mathbb{R}^{N}: f(x) \neq 0\right\}\right)<\infty$. Então, para todo $\varepsilon>0$, existe uma $\varphi \in C_{c}\left(\mathbb{R}^{N}\right)$ tal que:

$$
\mu\left(\left\{x \in \mathbb{R}^{N}: \varphi(x) \neq f(x)\right\}\right)<\varepsilon .
$$

Além disso, se $f$ é limitada, então podemos tomar $\varphi$ tal que:

$$
\|\varphi\|_{u} \leq\|f\|_{u}
$$

Demonstração. Ver [9], pág. 217, Teorema 7.10.

\subsubsection{O Dual de $C_{0}\left(\mathbb{R}^{N}\right)$}

O objetivo desta subseção é obter uma descrição completa do dual de $C_{0}\left(\mathbb{R}^{N}\right)$. Para isso, primeiramente definiremos o espaço $C_{0}\left(\mathbb{R}^{N}\right)$ e identificaremos os funcionais lineares contínuos e positivos sobre $C_{0}\left(\mathbb{R}^{N}\right)$ com a medidas finitas de Radon sobre $\mathbb{R}^{N}$. Posteriormente, definiremos o espaço das medidas de Radon com sinal sobre $\mathbb{R}^{N}$ para provarmos o resultado principal desta subseção. 
Definição 1.1.8. Seja $f \in C\left(\mathbb{R}^{N}\right)$. Se para todo $\varepsilon>0$, o conjunto:

$$
\left\{x \in \mathbb{R}^{N}:|f(x)| \geq \varepsilon\right\}
$$

for compacto, então dizemos que $f$ se anula no infinito. Com isso, definimos o seguinte conjunto:

$$
C_{0}\left(\mathbb{R}^{N}\right)=\left\{f \in C\left(\mathbb{R}^{N}\right): f \text { se anula no infinito }\right\}
$$

Além disso, com a norma

$$
\|f\|_{u}:=\sup \left\{|f(x)|: x \in \mathbb{R}^{N}\right\}
$$

temos que $C_{0}\left(\mathbb{R}^{N}\right)$ é um espaço de Banach.

Note que $C_{c}\left(\mathbb{R}^{N}\right) \subset C_{0}\left(\mathbb{R}^{N}\right)$, e ainda, toda $f \in C_{0}\left(\mathbb{R}^{N}\right)$ é limitada. Com isso, temos o seguinte resultado.

Proposição 1.1.9. $C_{0}\left(\mathbb{R}^{N}\right)$ é o fecho de $C_{c}\left(\mathbb{R}^{N}\right)$ na métrica uniforme.

Demonstração. Ver [9], pág. 132, Proposição 4.35.

Com a proposição acima, temos que dada $\mu$ uma medida de Radon, então o funcional

$$
I(f)=\int f d \mu
$$

onde $f \in C_{c}\left(\mathbb{R}^{N}\right)$, se estenderá continuamente a $C_{0}\left(\mathbb{R}^{N}\right)$ se, e somente se, $I$ for limitado com respeito a norma uniforme.

Proposição 1.1.10. O funcional I é contínuo se, e somente se, $\mu\left(\mathbb{R}^{N}\right)<\infty$.

Demonstração. Como caso particular de (1.1), temos a seguinte igualdade

$$
\mu\left(\mathbb{R}^{N}\right)=\sup \left\{\int f d \mu: f \in C_{c}\left(\mathbb{R}^{N}\right), \text { onde } 0 \leq f \leq 1\right\}
$$

e, ainda, do fato de $\left|\int f d \mu\right| \leq \int|f| d \mu$, obtemos:

$$
\mu\left(\mathbb{R}^{N}\right) \leq \sup \left\{\left|\int f d \mu\right|:\|f\|_{u}=1\right\} \quad \text { e } \quad\|I\|=\mu\left(\mathbb{R}^{N}\right)
$$

Portanto, $I$ será contínuo se, e somente se, $\mu\left(\mathbb{R}^{N}\right)<\infty$. 
Com isso, identificamos os funcionais lineares contínuos e positivos sobre $C_{0}\left(\mathbb{R}^{N}\right)$ com as medidas finitas de Radon sobre $\mathbb{R}^{N}$.

O próximo resultado nos diz que os funcionais lineares sobre $C_{0}\left(\mathbb{R}^{N}\right)$ tem uma certa decomposição de Jordan.

Lema 1.1.11. Seja $I \in C_{0}\left(\mathbb{R}^{N}\right)^{\prime}$. Então existem funcionais lineares positivos $I^{+}, I^{-} \in C_{0}\left(\mathbb{R}^{N}\right)^{\prime}$, tais que:

$$
I=I^{+}-I^{-} .
$$

Demonstração. Para demonstrarmos este lema, precisamos definir primeiro $I^{+}$. Para o que segue, denotaremos por $C_{0}\left(\mathbb{R}^{N},[0, \infty)\right)$ o seguinte conjunto:

$$
C_{0}\left(\mathbb{R}^{N},[0, \infty)\right)=\left\{f: \mathbb{R}^{N} \longrightarrow[0, \infty): f \in C_{0}\left(\mathbb{R}^{N}\right)\right.
$$

Assim, se $f \in C_{0}\left(\mathbb{R}^{N},[0, \infty)\right)$, então definimos:

$$
I^{+}(f)=\sup \left\{I(g): g \in C_{0}\left(\mathbb{R}^{N}, \mathbb{R}\right) \text { e } 0 \leq g \leq f\right\}
$$

Note que $I^{+}(f) \geq 0$. Para ver isso, basta fazer $g=0$. Além disso, pela desigualdade:

$$
|I(g)| \leq\left\|I \left|\|g\|_{u} \leq\|I \mid\| f \|_{u}\right.\right.
$$

sempre que $0 \leq g \leq f$, obtemos:

$$
0 \leq I^{+}(f) \leq\|I\|\|f\|_{u}
$$

Agora, vamos verificar que $I^{+}$é a restrição a $C_{0}\left(\mathbb{R}^{N},[0, \infty)\right)$ de um funcional linear. Observe que $I^{+}(c f)=c I^{+}(f)$, para todo $c \geq 0$.

Sejam $g_{1}, g_{2} \in C_{0}\left(\mathbb{R}^{N}\right)$ e $f_{1}, f_{2} \in C_{0}\left(\mathbb{R}^{N},[0, \infty)\right)$ arbitrárias tais que $0 \leq g_{1} \leq f_{1}$ e $0 \leq$ $g_{2} \leq f_{2}$. Somando termo a termo as desigualdades, obtemos $0 \leq g_{1}+g_{2} \leq f_{1}+f_{2}$, e portanto, $I^{+}\left(f_{1}+f_{2}\right) \geq I\left(g_{1}\right)+I\left(g_{2}\right)$. Logo,

$$
I^{+}\left(f_{1}+f_{2}\right) \geq I^{+}\left(f_{1}\right)+I^{+}\left(f_{2}\right) .
$$

Por outro lado, supondo que $0 \leq g \leq f_{1}+f_{2}$ e definindo $g_{1}=\min \left(g, f_{1}\right)$ e $g_{2}=g-g_{1}$, obtemos as inequações $0 \leq g_{1} \leq f_{1}$ e $0 \leq g_{2} \leq f_{2}$. Portanto, concluímos que $g=g_{1}+g_{2}$ e $I(g)=I\left(g_{1}\right)+I\left(g_{2}\right) \leq I^{+}\left(f_{1}\right)+I^{+}\left(f_{2}\right)$. Como $g$ é arbitrária, segue que:

$$
I^{+}\left(f_{1}+f_{2}\right) \leq I^{+}\left(f_{1}\right)+I^{+}\left(f_{2}\right)
$$


Logo, $I^{+}\left(f_{1}+f_{2}\right)=I^{+}\left(f_{1}\right)+I^{+}\left(f_{2}\right)$, para $f_{1}, f_{2} \in C_{0}\left(\mathbb{R}^{N},[0, \infty)\right)$.

Agora, considere $f \in C_{0}\left(\mathbb{R}^{N}\right)$. Então, temos que as partes positiva e negativa $f^{+}, f^{-}$de $f$ pertencem a $C_{0}\left(\mathbb{R}^{N},[0, \infty)\right)$. Assim, é natural definirmos:

$$
I^{+}(f)=I^{+}\left(f^{+}\right)-I^{+}\left(f^{-}\right)
$$

Afirmação 1.1.1. $I^{+}$é linear.

De fato, sejam $f, g \in C_{0}\left(\mathbb{R}^{N}\right)$ e $c \in \mathbb{R}$. Note que $I^{+}(c f)=c I^{+}(f)$. Fazendo a decomposição das funções $f, g$ e $f+g$, temos

$$
(f+g)^{+}-(f+g)^{-}=f+g=f^{+}-f^{-}+g^{+}-g^{-} .
$$

Logo,

$$
(f+g)^{+}+f^{-}+g^{-}=(f+g)^{-}+f^{+}+g^{+} .
$$

Assim, calculando $I^{+}$em cada uma das funções acima e reagrupando, obtemos:

$$
I^{+}\left[(f+g)^{+}\right]-I^{+}\left[(f+g)^{-}\right]=I^{+}\left(f^{+}\right)-I^{+}\left(f^{-}\right)+I^{+}\left(g^{+}\right)-I^{+}\left(g^{-}\right) .
$$

Logo,

$$
I^{+}(f+g)=I^{+}(f)+I^{+}(g)
$$

Afirmação 1.1.2. $I^{+}$é contínuo.

De fato, temos que:

$$
\left|I^{+}(f)\right| \leq \max \left\{I^{+}\left(f^{+}\right), I^{+}\left(f^{-}\right)\right\} \leq\|I\| \max \left\{\left\|f^{+}\right\|\left\|_{u},\right\| f^{-} \|_{u}\right\}=\|I\|\|f\|_{u} .
$$

Em particular, $\left\|I^{+}\right\| \leq\|I\|$.

Finalmente, definimos $I^{-}=I-I^{+}$. Então $I^{-} \in C_{0}\left(\mathbb{R}^{N}\right)^{\prime}$, e pela definição de $I^{+}$vemos que $I^{+}$e $I^{-}$são positivos. Portanto, segue o lema.

Pelos Teorema 1.1.6 e Lema 1.1.11, concluímos que para cada $I \in C_{0}\left(\mathbb{R}^{N}\right)^{\prime}$, existem duas medidas de Radon $\mu_{1}$ e $\mu_{2}$ tais que:

$$
I(f)=\int f d \mu, \quad \text { onde } \mu=\left(\mu_{1}-\mu_{2}\right) .
$$

Para finalmente demonstrarmos o resultado principal desta subseção abaixo, definiremos o espaço das medidas de Radon com sinal. 
Definição 1.1.12. Dizemos que $\mu$ é uma medida de Radon com sinal, quando $\mu$ é uma medida de Borel finita com sinal cujas variações positiva e negativa são medidas de Radon.

Denotaremos o espaço das medidas de Radon finitas com sinal sobre $\mathbb{R}^{N}$ por $M\left(\mathbb{R}^{N}\right)$. Para $\mu \in M\left(\mathbb{R}^{N}\right)$ com a variação total $|\mu|$ de $\mu$, definimos:

$$
\|\mu\|=|\mu|\left(\mathbb{R}^{N}\right) .
$$

Note que $\|\mu\|$ é finita, pois $\mu$ é limitada.

De maneira análoga, definimos o espaço das medidas de Radon finitas com sinal sobre $\Omega$ por $M(\Omega)$.

Com a definição acima, temos o seguinte resultado.

Proposição 1.1.13. $M\left(\mathbb{R}^{N}\right)$ é um espaço vetorial real $e$

$$
\mu \mapsto\|\mu\| \quad \text { é uma norma sobre } M\left(\mathbb{R}^{N}\right) \text {. }
$$

Demonstração. Ver [9], pág. 222, Proposição 7.16.

O resultado a seguir, nos permite identificar $C_{0}\left(\mathbb{R}^{N}\right)^{\prime}$ com o espaço $M\left(\mathbb{R}^{N}\right)$ e tal resultado é de extrema importância para se definir a topologia fraca em $M\left(\mathbb{R}^{N}\right)$.

Teorema 1.1.14 (Representação de Riez). Dada uma medida $\mu \in M\left(\mathbb{R}^{N}\right)$, consideremos o funcional:

$$
I_{\mu}(f)=\int f d \mu, \quad \text { onde } f \in C_{0}\left(\mathbb{R}^{N}\right) .
$$

A aplicação $\mu \mapsto I_{\mu}$ é um isomorfismo isométrico de $M\left(\mathbb{R}^{N}\right)$ em $C_{0}\left(\mathbb{R}^{N}\right)^{\prime}$.

Demonstração. Sabemos que todo funcional $I \in C_{0}\left(\mathbb{R}^{N}\right)^{\prime}$ é da forma $I_{\mu}$ (veja o comentário logo após o Lema 1.1.11). Com isso, já temos que a aplicação $\mu \mapsto I_{\mu}$ é sobrejetora.

Seja $\mu \in M\left(\mathbb{R}^{N}\right)$, então temos que (veja [9],Proposição 3.13 item c, pág. 94)

$$
\left|\int f d \mu\right| \leq \int|f| d|\mu| \leq\|f\|_{u}\|\mu\|
$$

e $\operatorname{assim} I_{\mu} \in C_{0}\left(\mathbb{R}^{N}\right)^{\prime}$ com norma $\left\|I_{\mu}\right\| \leq\|\mu\|$.

Além disso, definindo a derivada de Radon-Nikodym $h=\frac{d \mu}{d|\mu|}$, segue que $|h|=1$ (veja [9], Proposição 3.13, item b, pág.94). Então, pelo Teorema de Lusin aplicado a medida $|\mu|$, para todo $\varepsilon>0$, existirá uma função $f \in C_{c}\left(\mathbb{R}^{N}\right)$ satisfazendo:

$$
\|f\|_{u}=1 \text { e } f=\bar{h} \text { exceto em um conjunto } E \operatorname{com}|\mu|(E)<\varepsilon \text {. }
$$


Assim, utilizando $\bar{h} \in L^{1}(\mu)$ (pois $\mu\left(\mathbb{R}^{N}\right)<\infty$ ) e a Regra da Cadeia, temos:

$$
\|\mu\|=\int|h|^{2} d|\mu|=\int \bar{h} d \mu=\int_{E} \bar{h} d \mu+\int_{E^{c}} f d \mu \leq \int_{E} d|\mu|+|| I_{\mu}\|<\varepsilon+\| I_{\mu} \| .
$$

Como $\varepsilon$ é arbitrário, temos que $\|\mu\| \leq\left\|I_{\mu}\right\|$. Portanto, a aplicação é uma isometria sobrejetora.

Com a identificação do espaço $M\left(\mathbb{R}^{N}\right)$ com o espaço dual $C_{0}\left(\mathbb{R}^{N}\right)^{\prime}$ vamos definir uma topologia nesse espaço que é induzida por essa identificação.

Definição 1.1.15. A topologia fraca em $M\left(\mathbb{R}^{N}\right)=C_{0}\left(\mathbb{R}^{N}\right)^{\prime}$ é definida por:

$$
\mu_{\alpha} \longrightarrow \mu \Leftrightarrow \int f d \mu_{\alpha} \longrightarrow \int f d \mu
$$

para toda $f \in C_{0}\left(\mathbb{R}^{N}\right)$. Chamamos tal topologia de topologia vaga.

Vale observar que de maneira inteiramente análoga ao Teorema 1.1.14, pode-se mostrar que, dado $\Omega \subset \mathbb{R}^{N}$, um aberto, temos:

$$
M(\Omega) \cong C_{0}(\Omega)^{\prime}
$$

via o mesmo isomorfismo isométrico. Isto motiva a seguinte definição.

Definição 1.1.16 (Convergência Vaga em $M(\Omega)$ ). A topologia fraca em $M(\Omega)=C_{0}(\Omega)^{\prime}$ é definida por

$$
\mu_{\alpha} \longrightarrow \mu \Leftrightarrow \int f d \mu_{\alpha} \longrightarrow \int f d \mu
$$

para toda $f \in C_{0}(\Omega)$.

Também observamos que se $f_{n} \rightarrow f$ em $L^{r}(\Omega)$ e $r>1$, então $f_{n} \rightarrow f$ vagamente em $M(\Omega)$. De fato, basta notar que

$$
C_{0}(\Omega) \hookrightarrow L^{r^{\prime}}(\Omega)
$$

Um bom motivo para usarmos a topologia vaga é porque argumentos de convergência fraca para $L^{p}(\Omega)$ geralmente falham quando $p=1$, pois $L^{1}(\Omega)$ não é o dual de $L^{\infty}(\Omega)$, mas podemos obter bons resultados usando a topologia vaga quando identificamos $L^{1}(\Omega)$ como um subespaço de $M(\Omega)$.

Para fazermos tal identificação, seja $\Omega \subseteq \mathbb{R}^{N}$. Dada $v \in L^{1}(\Omega)$, (aqui consideramos a medida de Lebesgue) associamos a seguinte aplicação

$$
T_{v}(\Omega)=\int_{\Omega} v
$$


Note que $T_{v}$ é uma medida de Radon, e além disso,

$$
\left\|T_{v}\right\|_{M(\Omega)}=\left|T_{v}\right|(\Omega)=\int_{\Omega}|v|=\|v\|_{L^{1}}
$$

e assim, temos que $L^{1}(\Omega)$ está imerso isometricamente em $M(\Omega)$.

Um fato importante é que $L^{1}(\Omega)$ é denso "vagamente" (denso considerando a topologia vaga) em $M(\Omega)$, onde $\Omega$ pode ser o próprio $\mathbb{R}^{N}$. Na próxima subseção, definiremos e apresentaremos resultados para demonstrar tal fato.

Observação 1.1.17. De agora em diante, denotaremos por $M(\Omega)$ o espaço das medidas de Radon reais sobre $\Omega$.

\subsubsection{Densidade Vaga de $L^{1}\left(\mathbb{R}^{N}\right)$ em $M\left(\mathbb{R}^{N}\right)$}

Nesta subseção, vamos primeiramente definir e enunciar alguns resultados sobre convolução entre duas funções mensuráveis. Posteriormente, definiremos o produto de medidas de Radon com sinal e trataremos de resultados sobre o conceito de convolução de duas medidas. Por fim, demonstraremos o resultado de densidade vaga de $L^{1}\left(\mathbb{R}^{N}\right)$ em $M\left(\mathbb{R}^{N}\right)$. Esse estudo se faz necessário para o Capítulo 3, onde precisaremos aproximar uma função $f \in M(\Omega)$ por uma sequência de funções que estão em $L^{1}(\Omega)$.

Definição 1.1.18. Sejam $f$ e $g$ duas funções mensuráveis em $\mathbb{R}^{N}$. Definimos a convolução de $f$ e g por:

$$
f * g(x)=\int_{\mathbb{R}^{N}} f(x-y) g(y) d y
$$

para todo $x$ tal que a integral acima exista.

Para que $f * g$ esteja bem definida, podemos impor várias condições sobre $f$ e $g$. Por exemplo, se $g \in L_{l o c}^{1}\left(\mathbb{R}^{N}\right)$ e $f$ é limitada com suporte compacto, então $f * g$ existe.

Na próxima proposição, vamos apresentar algumas propriedades sobre convolução.

Proposição 1.1.19. Assuma que todas as integrais abaixo sejam finitas. Então:

(a) $f * g=g * f$.

(b) $(f * g) * h=f *(g * h)$.

(c) Seja $z \in \mathbb{R}^{N}$, então $\tau_{z}(f * g)=\left(\tau_{z} f\right) * g=f *\left(\tau_{z} g\right)$, onde $\tau_{z} f(x)=f(x-z)$.

(d) Seja A o fecho de $\{x+y: x \in \operatorname{supp}(f), y \in \operatorname{supp}(g)\}$, então supp $(f * g) \subset A$. 
Demonstração. Ver [9], pág. 240, Proposição 8.6.

A proposição a seguir contém resultados básicos de convoluções para funções em $L^{p}$.

Proposição 1.1.20. Sejam $f \in L^{p}$ e $g \in L^{q}$, onde $\frac{1}{p}+\frac{1}{q}=1$. Então:

(a) $f * g(x)$ existe para todo $x$.

(b) $f * g$ é limitada e uniformemente contínua.

(c) $\|f * g\|_{u} \leq\|f\|_{L^{p}}\|g\|_{L^{q}}$.

(d) Se $1<p<\infty$, então $f * g \in C_{0}\left(\mathbb{R}^{N}\right)$.

Demonstração. Ver [9], pág. 241, Proposição 8.8.

Seja $\phi$ uma função em $\mathbb{R}^{N}$ e $t>0$. Definimos:

$$
\phi_{t}(x)=t^{-n} \phi\left(\frac{x}{t}\right) .
$$

Se $\phi \in L^{1}$, então pelo Teorema da Mudança de Variável (veja [9], pág. 74) temos que a integral $\int \phi_{t}$ não depende de $t$, isto é,

$$
\int_{\mathbb{R}^{N}} \phi_{t}=\int_{\mathbb{R}^{N}} t^{-n} \phi\left(\frac{x}{t}\right) d x=\int_{\mathbb{R}^{N}} \phi(z) d z,
$$

onde $z=\frac{x}{t}$.

O teorema a seguir será de extrema importância na demonstração do principal resultado desta subseção.

Teorema 1.1.21. Seja $\phi \in L^{1}$ tal que $\int \phi(x) d x=a$. Então:

(a) Se $f \in L^{p}$, para $1 \leq p<\infty$, então $f * \phi_{t} \rightarrow$ af em $L^{p}$, quando $t \rightarrow 0$.

(b) Se $f$ é limitada e uniformemente contínua, então $f * \phi_{t} \rightarrow$ af uniformemente, quando $t \rightarrow 0$.

(c) Se $f \in L^{\infty}$ e $f$ é contínua em um aberto $U$, então $f * \phi_{t} \rightarrow$ af uniformemente em todo subconjunto compacto de $U$, quando $t \rightarrow 0$.

Demonstração. Ver [9], pág. 242, Teorema 8.14.

Agora, vamos definir o que seria uma medida de Radon com sinal no espaço produto cartesiano $\mathbb{R}^{N} \times \mathbb{R}^{N}$ e a convolução de duas medidas $\mu$ e $\nu$, onde $\mu, \nu \in M\left(\mathbb{R}^{N}\right)$, com o intuito de obtermos os resultados necessários para a prova da densidade vaga de $L^{1}\left(\mathbb{R}^{N}\right)$ em $\mathbb{R}^{N}$. A definição da medida de Radon com sinal no produto cartesiano pode ser feita utilizando produto de medidas positivas através das derivadas de Radon-Nikodym. 
Definição 1.1.22. Sejam $\mu, \nu \in M\left(\mathbb{R}^{N}\right)$, definimos a medida produto $\mu \times \nu \in M\left(\mathbb{R}^{N} \times \mathbb{R}^{N}\right)$ por:

$$
d(\mu \times \nu)(x, y)=\frac{d \mu}{d|\mu|}(x) \frac{d \nu}{d|\nu|}(y) d(|\mu| \times|\nu|)(x, y) .
$$

Definição 1.1.23. Sejam $\mu, \nu \in M\left(\mathbb{R}^{N}\right)$, definimos a convolução $\mu * \nu \in M\left(\mathbb{R}^{N}\right)$ por:

$$
\mu * \nu(E)=\mu \times \nu(E)=\iint \mathcal{X}_{E}(x+y) d \mu(x) d \nu(y) .
$$

Abaixo, apresentamos algumas propriedades básicas da convolução de duas medidas em $M\left(\mathbb{R}^{N}\right)$

Proposição 1.1.24. Sejam $\mu, \nu \in M\left(\mathbb{R}^{N}\right)$.

(a) Convolução de medidas é comutativa e associativa.

(b) Seja h uma função limitada Borel mensurável, então:

$$
\int h d(\mu \times \nu)=\iint h(x+y) d \mu(x) d \nu(y) .
$$

(c) $\|\mu * \nu\| \leq\|\mu\|\|\nu\|$.

(d) Se $d \mu=f d m$ e $d \nu=g d m$, então $d(\mu * \nu)=(f * g) d m$.

Demonstração. Ver [9], pág. 270, Proposição 8.48.

Observação 1.1.25. Note que o item (d) da Proposição acima nos diz que em $L^{1}$ a definição de convolução entre medidas coincide com a definição usual de convolução.

Quando temos uma função $f$ em $L^{p}$, é importante obtermos algumas propriedades quando fazemos a convolução de $f$ com uma medida $\mu$ em $M\left(\mathbb{R}^{N}\right)$. O próximo teorema nos dá três propriedades dessa convolução.

Teorema 1.1.26. Sejam $f \in L^{p}\left(\mathbb{R}^{N}\right)$, para $1 \leq p \leq \infty$, e $\mu \in M\left(\mathbb{R}^{N}\right)$. Então:

(a) A integral $f * \mu(x)=\int f(x-y) d \mu(y)$ existe para $x$ q.t.p. em $\mathbb{R}^{N}$.

(b) $f * \mu \in L^{p}$.

(c) $\|f * \mu\|_{L^{p}} \leq\|f\|_{L^{p}}\|\mu\|$.

Demonstração. Ver [9], pág. 271, Proposição 8.49.

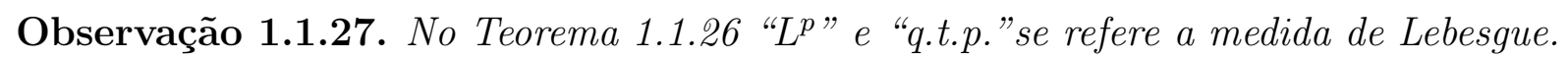

Por fim, estamos preparados para mostrar o principal resultado desta subseção. 
Teorema 1.1.28. $L^{1}\left(\mathbb{R}^{N}\right)$ é denso vagamente em $M\left(\mathbb{R}^{N}\right)$.

Demonstração. Seja $\mu\left(\mathbb{R}^{N}\right)$. Para provarmos este teorema, devemos construir uma sequência em $L^{1}\left(\mathbb{R}^{N}\right)$ que converge no sentido vago para $\mu$.

Seja $\phi \in C_{c}^{\infty}\left(\mathbb{R}^{N}\right)$ fixada, com $\int_{\mathbb{R}^{N}} \phi(x) d x=1$. Então para todo $t>0$ pelo Teorema 1.1.26, temos:

$$
\phi_{t} * \mu \in L^{1}\left(\mathbb{R}^{N}\right) .
$$

Sabemos que toda função em $L^{1}\left(\mathbb{R}^{N}\right)$ define uma medida de Radon (veja o comentário após o Teorema 1.1.14, pág. 12), então vamos escrever $\mu_{t} \in M\left(\mathbb{R}^{N}\right)$ para a medida que corresponde a $\phi_{t} * \mu$, ou seja, $d \mu_{t}=\left(\phi_{t} * \mu\right) d m$. Agora, seja $g \in C_{0}\left(\mathbb{R}^{N}\right)$ fixada. Pelo Teorema de Fubini para a medida produto $\mu \times m$, onde $m$ é a medida de Lebesgue em $\mathbb{R}^{N}$, temos que:

$$
\begin{aligned}
\int_{\mathbb{R}^{N}} g d \mu_{t}=\int_{\mathbb{R}^{N}} g(x)\left(\phi_{t} * \mu\right)(x) d x & =\int_{\mathbb{R}^{N}}\left(\int_{\mathbb{R}^{N}} g(x) \phi_{t}(x-y) d \mu(y)\right) d x \\
& =\int_{\mathbb{R}^{N}} \int_{\mathbb{R}^{N}} g(x) \phi_{t}(x-y) d x d \mu(y) \\
& =\int_{\mathbb{R}^{N}} g * \tilde{\phi}_{t}(y) d \mu(y) .
\end{aligned}
$$

Observe que a função $(x, y) \mapsto g(x) \phi_{t}(x-y)$ é contínua, pois é produto de funções contínuas. Assim, temos que $g(x) \phi_{t}(x-y)$ é $\mathcal{B}_{\mathbb{R}^{N}} \otimes \mathcal{B}_{\mathbb{R}^{N}}$-mensurável. Note também que:

$$
\begin{aligned}
\int_{\mathbb{R}^{N}}\left(\int_{\mathbb{R}^{N}}\left|g(x) \phi_{t}(x-y)\right| d x\right) d|\mu|(y) & =\int_{\mathbb{R}^{N}}\left(\int_{K}\left|g(x) \phi_{t}(x-y)\right| d x\right) d|\mu|(y) \\
& \leq \int_{\mathbb{R}^{N}}|| g\left\|_{L^{1}}|| \phi_{t}\right\|_{u} d|\mu|=\|g\|_{L^{1}}\left\|\phi_{t}\right\|\left\|_{u}\right\| \mu \|<\infty,
\end{aligned}
$$

onde $K$ é o compacto tal que $\phi_{t}(x-y)$ é diferente de zero.

Afirmação 1.1.3. $g * \tilde{\phi}_{t} \in C_{0}\left(\mathbb{R}^{N}\right)$ para cada $t>0$.

De fato, mais geralmente provamos que para $\psi \in C_{0}\left(\mathbb{R}^{N}\right)$ e $\varphi \in C_{c}\left(\mathbb{R}^{N}\right)$ temos $\psi * \varphi \in$ $C_{0}\left(\mathbb{R}^{N}\right)$. Primeiro, pela Proposição 1.1.20, pág. 14, temos que $\psi * \varphi$ é limitada e uniformemente contínua e $\|\psi * \varphi\|_{u} \leq\|\varphi\|_{L^{1}}\|\psi\|_{u}$. Agora, tomamos uma sequência $\psi_{1}, \psi_{2}, \ldots \in C_{c}\left(\mathbb{R}^{N}\right)$ tal que $\left\|\psi-\psi_{k}\right\| \longrightarrow 0$ (Proposição 1.1.9, pág. 8). Então $\psi_{k} * \varphi \in C_{c}\left(\mathbb{R}^{N}\right)$ para cada $k$, pela Proposição 1.1.19 item (d), pág. 14, e

$$
\left\|\psi_{k} * \varphi-\psi * \varphi\right\|_{u}=\left\|\left(\psi_{k}-\psi\right) * \varphi\right\|_{u} \leq\|\varphi\|_{L^{1}}\left\|\psi_{k}-\psi\right\|_{u} \longrightarrow 0 .
$$


Portanto, $\psi * \varphi$ pertence ao fecho de $C_{c}\left(\mathbb{R}^{N}\right)$ na métrica uniforme, ou seja, $\psi * \varphi \in C_{0}\left(\mathbb{R}^{N}\right)$ (Proposição 1.1.9, pág. 8). Logo, a afirmação está provada.

Agora, pelo Teorema 1.1.21 item (b), pág. 15, temos $\left\|g * \tilde{\phi}_{t}-g\right\|_{u} \longrightarrow 0$, quando $t \longrightarrow 0$. Portanto, como $f \mapsto \int_{\mathbb{R}^{N}} f d \mu$ é um funcional linear limitado em $C_{0}\left(\mathbb{R}^{N}\right)$, segue que:

$$
\int_{\mathbb{R}^{N}} g * \phi_{t}(y) d \mu(y) \longrightarrow \int_{\mathbb{R}^{N}} g d \mu
$$

quando $t \longrightarrow 0$. Assim

$$
\int_{\mathbb{R}^{N}} g d \mu_{t} \longrightarrow \int_{\mathbb{R}^{N}} g d \mu
$$

quando $t \longrightarrow 0$ e para toda $g \in C_{0}\left(\mathbb{R}^{N}\right)$. Então, concluímos que a sequência $\left\{\mu_{\frac{1}{j}}\right\}_{j=1}^{\infty}$ tende vagamente para $\mu$. Recordando que cada $\mu_{\frac{1}{j}}$ corresponde a uma função (ou: é uma) em $L^{1}\left(\mathbb{R}^{N}\right.$ ) temos que $L^{1}\left(\mathbb{R}^{N}\right)$ é denso vagamente em $M\left(\mathbb{R}^{N}\right)$.

Corolário 1.1.29. Seja $\Omega \subset \mathbb{R}^{N}$ um aberto. Então $L^{1}(\Omega)$ é denso vagamente em $M(\Omega)$

Demonstração. Seja $\mu \in M(\Omega)$. Definimos $\bar{\mu}$ por:

$$
\bar{\mu}= \begin{cases}\mu & \text { em } \Omega \\ 0 & \text { em } \mathbb{R}^{N} \backslash \Omega\end{cases}
$$

Note que $\bar{\mu} \in M\left(\mathbb{R}^{N}\right)$. Pelo Teorema 1.1.28, existe uma sequência $\left(f_{n}\right)$ em $L^{1}\left(\mathbb{R}^{N}\right)$ que converge para $\bar{\mu}$ no sentido vago. Como $f_{n} \in L^{1}\left(\mathbb{R}^{N}\right)$, para todo $n$, temos que $f_{n} \in L^{1}(\Omega)$, para todo $n$.

Assim, temos uma sequência $\left(f_{n}\right)$ em $L^{1}(\Omega)$ que converge vagamente para $\bar{\mu}$. Consequentemente, $f_{n}$ converge vagamente para $\mu$.

Corolário 1.1.30. Dado $r \geq 1$, segue que $L^{r}(\Omega)$ é denso vagamente em $M(\Omega)$.

Demonstração. Primeiro, note que se $u_{n} \longrightarrow u$ em $L^{r}(\Omega)$, então $u_{n} \longrightarrow u$ vagamente em $M(\Omega)$.

De fato, pela Definição 1.1.16, basta mostrarmos que:

$$
\int_{\Omega} f u_{n} \longrightarrow \int_{\Omega} f u, \quad \forall f \in C_{0}(\Omega) .
$$

Porém, por Hölder:

$$
\begin{aligned}
\left|\int_{\Omega} f u_{n}-f u\right| & \leq\|f\|_{L^{r^{\prime}}}\left\|u_{n}-u\right\|_{L^{r}} \\
& \leq \operatorname{med}(\Omega)\|f\|_{L^{\infty}}\left\|u_{n}-u\right\|_{L^{r}} \longrightarrow 0 .
\end{aligned}
$$


Logo, $u_{n} \longrightarrow u$ vagamente em $M(\Omega)$.

Agora, dada $\mu \in M(\Omega)$, consideremos $\left(v_{n}\right) \subset L^{1}(\Omega)$ tal que:

$$
\lim _{n \rightarrow \infty} \int_{\Omega} f v_{n}=\int_{\Omega} f d \mu, \quad \forall f \in C_{0}(\Omega) .
$$

Como $L^{r}(\Omega)$ é denso em $L^{1}(\Omega)$, segue que para cada $n \in \mathbb{N}$, existe $u_{n} \in L^{r}(\Omega)$ tal que:

$$
\left\|u_{n}-v_{n}\right\|_{L^{1}} \leq \frac{1}{n}
$$

Assim, dada $f \in C_{0}(\Omega)$ temos:

$$
\begin{aligned}
\left|\int_{\Omega} f d \mu-\int_{\Omega} f u_{n}\right| & \leq\left|\int_{\Omega} f d \mu-\int_{\Omega} f v_{n}\right|+\left|\int_{\Omega} f v_{n}-\int_{\Omega} f u_{n}\right| \\
& \leq\left|\int_{\Omega} f d \mu-\int_{\Omega} f v_{n}\right|+\frac{1}{n}
\end{aligned}
$$

Logo

$$
\lim _{n \rightarrow \infty}\left|\int_{\Omega} f d \mu-\int_{\Omega} f u_{n}\right| \leq \lim _{n \rightarrow \infty}\left|\int_{\Omega} f d \mu-\int_{\Omega} f v_{n}\right|=0
$$

e portanto, $u_{n} \longrightarrow u$ vagamente em $M(\Omega)$.

\subsubsection{Distribuições}

Nesta subseção, definiremos o conceito de distribuição em $\Omega$ apenas com o intuito de mostrar que convergência vaga implica em convergência em distribuição. Tal resultado será usado no Capítulo 3. Para mais detalhes sobre este tema, veja Medeiros [18].

Definição 1.1.31. Considere $\left(f_{n}\right) \subset C_{c}^{\infty}(\Omega)$. Dizemos que:

(a) $f_{n} \rightarrow 0$ desde que:

(i) Exista $K \subset \Omega$, compacto tal que supp $f_{n} \subset K, \forall n \in \mathbb{N}$.

(ii) Para cada $\alpha$ multi-indice $D^{\alpha} f_{n} \rightarrow 0$ uniformemente em $K$.

(b) Dizemos que $f_{n} \rightarrow f$ desde que $\left(f_{n}-f\right) \rightarrow 0$.

O espaço denotado por $D(\Omega)$ das funções em $C_{c}^{\infty}(\Omega)$ com a noção da convergência definida acima é chamado o espaço das funções teste.

Definição 1.1.32. Dizemos que $T: D(\Omega) \longrightarrow \mathbb{R}$ é uma distribuição se:

(a) Té linear;

(b) T é contínuo no zero em $D(\Omega)$, ou seja, $T\left(f_{n}\right) \rightarrow 0$, se $n \rightarrow \infty$, sempre que $f_{n} \rightarrow 0$ em $D(\Omega)$. 
O conjunto de todas as distribuições definidas em $\Omega$ é denotado por $D^{\prime}(\Omega)$. No espaço $D^{\prime}(\Omega)$, temos a seguinte noção de convergência.

Definição 1.1.33 (Convergência em $D^{\prime}(\Omega)$ ). Dizemos que

(a) $T_{n} \rightarrow 0$ em $D^{\prime}(\Omega)$ quando $n \rightarrow \infty$, se

$$
T_{n}(\phi) \rightarrow 0 \text { em } \mathbb{R}, \text { quando } n \rightarrow \infty, \forall \phi \in D(\Omega) .
$$

(b) $T_{n} \rightarrow T$ em $D^{\prime}(\Omega)$ se

$$
\left(T_{n}-T\right) \rightarrow 0 \text { quando } n \rightarrow \infty, \text { em } D^{\prime}(\Omega)
$$

Segue diretamente da definição acima que convergência em distribuição é única. Um fato importante é que uma medida $\mu$ em $M(\Omega)$ pode ser vista como uma distribuição em $\Omega$.

Proposição 1.1.34. Seja $\mu \in M(\Omega)$. Então $\mu$ pode ser representada como uma distribuição, isto é, $\mu \in D^{\prime}(\Omega)$.

Demonstração. Seja $\phi \in D(\Omega)$. Definimos:

$$
(\mu, \phi)=\int_{\Omega} \phi d \mu
$$

Note que $(\mu, \phi)$ é um funcional linear contínuo. Agora, se $\phi_{n} \rightarrow 0$ em $D(\Omega)$, então existe $K \subset \Omega$, compacto, tal que supp $\phi_{n} \subset K$, para todo $n \in \mathbb{N}$ e

$$
D^{\alpha} \phi_{n} \rightarrow 0 \text { uniformemente em } K, \forall \alpha \text { multi-índice. }
$$

Em particular, $\phi_{n} \rightarrow 0$ uniformemente em $K$. Logo

$$
\lim _{n \rightarrow \infty}\left(\mu, \phi_{n}\right)=\lim _{n \rightarrow \infty} \int_{K} \phi_{n} d \mu \longrightarrow 0
$$

Portanto, $\mu \in D^{\prime}(\Omega)$.

Usando a mesma identificação, como corolário da Proposição 1.1.34, segue que $L^{p}(\Omega) \subset$ $D^{\prime}(\Omega)$. A noção de convergência no espaço $D^{\prime}(\Omega)$ é bem fraca. De fato, o próximo resultado nos mostra que até convergência vaga implica em convergência no espaço $D^{\prime}(\Omega)$.

Teorema 1.1.35. Seja $\left(\mu_{n}\right) \subset M(\Omega)$ tal que $\mu_{n} \rightarrow \mu$ vagamente em $M(\Omega)$. Então:

$$
\mu_{n} \rightarrow \mu \quad \text { em } D^{\prime}(\Omega) .
$$


Demonstração. Como por hipótese $\mu_{n} \rightarrow \mu$ vagamente em $M(\Omega)$, então pela definição:

$$
\int_{\Omega} \phi d \mu_{n} \longrightarrow \int_{\Omega} \phi d \mu
$$

para toda $\phi \in C_{0}(\Omega)$. Para mostrar que $\mu_{n} \rightarrow \mu$ em $D^{\prime}(\Omega)$, basta mostrarmos que, dado $\phi \in D(\Omega)$, então:

$$
\lim _{n \rightarrow \infty}\left(\mu_{n}, \phi\right)=(\mu, \phi) .
$$

A igualdade acima acontece se, e somente se,

$$
\int_{\Omega} \phi d \mu_{n} \longrightarrow \int_{\Omega} \phi d \mu
$$

Porém, como $D(\Omega) \subset C_{0}(\Omega)$ segue o resultado.

Observe que segue diretamente do Teorema 1.1.35, que dadas $\left(f_{n}\right) \subset L^{r}(\Omega)$, onde $r>1$ e $f \in L^{r}(\Omega)$, se

$$
f_{n} \rightarrow f \quad \text { em } L^{r}(\Omega)
$$

então vale que

$$
f_{n} \longrightarrow f \quad \text { em } D^{\prime}(\Omega)
$$

Isto é,

$$
L^{r}(\Omega) \hookrightarrow \hookrightarrow C_{0}(\Omega)
$$

\subsection{Espaços de Sobolev}

Usando o conceito de Distribuição, conseguimos generalizar a ideia de derivada para uma formulação mais fraca.

Seja $\Omega \subset \mathbb{R}^{N}$ um aberto, limitado e conexo.

Definição 1.2.1. Definimos o Espaço de Sobolev, denotado por $W^{1, p}(\Omega)$ como sendo:

$$
W^{1, p}(\Omega)=\left\{u: \Omega \longrightarrow \mathbb{R}: u, \frac{\partial u}{\partial x_{i}} \in L^{p}(\Omega), i=1, \ldots, N\right\} .
$$

Observe que $\frac{\partial u}{\partial x_{i}}$ se refere a derivada no sentido das distribuições.

Proposição 1.2.2. $\left(W^{1, p}(\Omega),\|\cdot\|_{W^{1, p}}\right)$, onde

$$
\|u\|_{W^{1, p}}=\left\{\begin{array}{l}
\left(\|u\|_{L^{p}}^{p}+\|\nabla u\|_{L^{p}}^{p}\right)^{1 / p}, 1 \leq p<\infty \\
\|u\|_{L^{\infty}}+\|\nabla u\|_{L^{\infty}}
\end{array}\right.
$$

é um espaço de Banach. Além disso, se $1<p<\infty$, é um espaço de Banach reflexivo. 
Demonstração. Ver [4], pág. 264, Proposição 9.1.

Das diversas propriedades interessantes destes espaços, nos restringiremos a enunciar o essencial para o presente texto.

Primeiramente, vejamos seu subespaço mais usual.

Definição 1.2.3. $W_{0}^{1, p}(\Omega)={\overline{C_{c}^{\infty}(\Omega)}}^{\|} \|_{W^{1, p}}$.

Com isso, temos o seguinte resultado.

Proposição 1.2.4. $W_{0}^{1, p}(\Omega)$ é um subespaço fechado de $W^{1, p}(\Omega)$. Em particular, $W_{0}^{1, p}(\Omega)$ é um espaço de Banach reflexivo se $1<p<\infty$.

Demonstração. Ver [4], pág. 287.

Um fato importante dos espaços $W_{0}^{1, p}(\Omega)$ é que podemos controlar as normas $L^{p}$ pelas normas das derivadas em certos casos.

Definição 1.2.5. Definimos o expoente crítico de Sobolev $p^{*}$, por:

$$
p^{*}=\frac{N p}{N-p} .
$$

Se $p=N$, então definimos $p^{*}=\infty$.

Observação 1.2.6. Por simplicidade, enunciaremos os próximos resultados somente para o caso que usaremos neste trabalho, isto é, quando $1 \leq p<N$.

Teorema 1.2.7 (Desigualdade de Poincaré). Seja $u \in W_{0}^{1, p}(\Omega)$, onde $1 \leq p<N$. Então existe uma constante $C=C(\Omega, p, q, N)>0$ tal que

$$
\|u\|_{L^{q}} \leq C\|\nabla u\|_{L^{p}}
$$

para todo $q \in\left[1, p^{*}\right]$.

Demonstração. Ver [8], pág. 265, Teorema 3.

Observamos que $\|\nabla u\|_{L^{p}}$ é uma norma em $W_{0}^{1, p}(\Omega)$, que é equivalente a norma $\|u\|_{W^{1, p}}$. Assim, definimos a seguinte norma em $W_{0}^{1, p}(\Omega)$

$$
\|u\|_{W_{0}^{1, p}}=\|\nabla u\|_{L^{p}} .
$$

Uma das propriedades mais usadas destes espaços é o de que estes melhoram a integrabilidade de seus membros. 
Teorema 1.2.8 (Imersão de Sobolev). Seja $\Omega \subset \mathbb{R}^{N}$ um aberto, limitado e conexo. Para $1 \leq p<N$, temos

$$
W_{0}^{1, p}(\Omega) \hookrightarrow L^{q}(\Omega)
$$

para todo $q \in\left[1, p^{*}\right]$.

Demonstração. Ver [8], pág. 270, Teorema 6.

Com algumas restrições no expoente, melhora-se o resultado de Imersão.

Teorema 1.2.9 (Rellich-Kondrachov). Seja $\Omega \subset \mathbb{R}^{N}$ um aberto, limitado e conexo. Para $1 \leq p<N$, temos

$$
W_{0}^{1, p}(\Omega) \hookrightarrow \hookrightarrow L^{q}(\Omega)
$$

para todo $q \in\left[1, p^{*}\right)$.

Demonstração. Ver [8], pág. 272, Teorema 1.

\subsection{Grau de Brouwer}

No presente texto, usaremos uma poderosa ferramenta para a resolução de equações não lineares em espaços de dimensão finita: O grau de Brouwer.

Para não fugirmos da temática proposta nesta dissertação, nos restringiremos a enunciar o resultado que nos interessa.

Seja $\phi: \Omega \subset \mathbb{R}^{N} \longrightarrow \mathbb{R}^{N}$ uma função contínua, $p \notin \phi(\partial \Omega)$. O grau de Brouwer $d(\phi, \Omega, p)$ é uma ferramenta que descreve o número de soluções para a equação:

$$
\phi(x)=p
$$

onde $\Omega$ é um aberto.

A seguir apresentaremos dois resultados que nos será útil para esta dissertação.

Teorema 1.3.1. Existe $d(\cdot, \Omega, p): C^{1}(\bar{\Omega}) \longrightarrow \mathbb{R}$ tal que se $p \notin \phi(\partial \Omega)$ e $d(\phi, \Omega, p) \neq 0$, então existe ao menos um $x \in \Omega$ tal que

$$
\phi(x)=p
$$

Demonstração. Ver [10], pág. 30, Teorema 2.1. 
Teorema 1.3.2 (Invariância Homotópica). Seja $H: \bar{\Omega} \times[0,1] \longrightarrow \mathbb{R}^{N}$. Considere $p \in \mathbb{R}^{N} e$ suponha que $p \notin H(\partial \Omega \times[0,1])$. Então

$$
d(H(\cdot, 0), \Omega, p)=d(H(\cdot, 1), \Omega, p)
$$

Demonstração. Ver [10], pág. 39, Teorema 2.11.

\subsection{Teoremas de Convergência}

Esta seção é baseada em [1] e [9]. Os resultados que apresentaremos são alguns dos principais da Teoria da Medida e Integração que posteriormente usaremos no decorrer deste trabalho.

Seja $(X, \mathcal{M}, \mu)$ um espaço de medida. Em toda essa seção, $\mu$ denotará uma medida positiva em $X$.

Lema 1.4.1 (Lema de Fatou). Seja $\left(f_{n}\right)$ uma sequência de funções mensuráveis com $f_{n}: X \rightarrow$ $[0, \infty]$, para todo $n \in \mathbb{N}$. Então

$$
\int \liminf _{n \rightarrow \infty} f_{n} d \mu \leq \liminf _{n \rightarrow \infty} \int f_{n} d \mu
$$

Demonstração. Ver [1], pág. 33, Lema 4.8.

Apresentaremos a seguir um dos mais importantes teoremas de convergência para funções integráveis.

Teorema 1.4.2 (Convergência Dominada de Lebesgue). Seja $\left(f_{n}\right)$ uma sequência de funções em $L^{1}(X, \mu)$ tal que converge q.t.p. para uma função $f$. Além disso, suponha que existe uma função $g$ em $L^{1}(X, \mu)$ tal que $\left|f_{n}\right| \leq g$, para todo $n \in \mathbb{N}$. Então $f \in L^{1}(X, \mu)$ e

$$
\lim _{n \rightarrow \infty} \int f_{n} d \mu=\int f d \mu .
$$

Demonstração. Ver [1], pág. 44, Teorema 5.6.

Com o intuito de generalizarmos o Teorema da Convergência Dominada de Lebesgue para os espaços $L^{p}(X, \mu)$, vamos definir o que seria a convergência de uma sequência de funções nesse espaço. 
Definição 1.4.3. Dizemos que uma sequência de funções $\left(f_{n}\right)$ em $L^{p}(X, \mu), 1 \leq p<\infty$, converge em $L^{p}(X, \mu)$ para $f \in L^{p}(X, \mu)$, se dado $\varepsilon>0$, existir $n_{0}(\varepsilon) \in \mathbb{N}$, tal que para todo $n \geq n_{0}(\varepsilon)$, temos:

$$
\left\|f_{n}-f\right\|_{L^{p}}=\left(\int\left|f_{n}-f\right|^{p} d \mu\right)^{\frac{1}{p}}<\varepsilon .
$$

A proposição a seguir é a versão do Teorema da Convergência Dominada de Lesbegue para os espaços $L^{p}(X, \mu)$.

Proposição 1.4.4. Seja $\left(f_{n}\right)$ uma sequência de funções mensuráveis em $L^{p}(X, \mu)$ que converge q.t.p. para $f$. Se existe uma $g \in L^{p}(X, \mu)$ tal que:

$$
\left|f_{n}(x)\right| \leq g(x), \quad x \in X, n \in \mathbb{N}
$$

então $f \in L^{p}(X, \mu)$ e $\left(f_{n}\right)$ converge em $L^{p}(X, \mu)$ para $f$.

Demonstração. Por hipótese $f_{n}(x) \rightarrow f(x)$ q.t.p. em $X$. Como a sequência de funções $\left(f_{n}\right)$ é mensurável, para todo $n \in \mathbb{N}$, segue que $f$ é mensurável. Ainda, uma vez que $\left|f_{n}(x)\right| \leq$ $g(x), \forall n \in \mathbb{N}$, então $|f(x)| \leq g(x)$ q.t.p. Além disso, como $g \in L^{p}(X, \mu)$, então temos $f \in L^{p}(X, \mu)$. Agora note que:

$$
\left|f_{n}(x)-f(x)\right|^{p} \leq[2 g(x)]^{p} \text {, q.t.p. }
$$

e como $\lim \left|f_{n}(x)-f(x)\right|^{p}=0$ q.t.p., e $[2 g(x)]^{p} \in L^{1}(X, \mu)$, segue do Teorema da Convergência Dominada de Lebesgue que:

$$
\lim \int\left|f_{n}-f\right|^{p} d \mu=0
$$

Portanto, $\left(f_{n}\right)$ converge em $L^{p}(X, \mu)$ para $f$.

O próximo teorema é uma espécie de recíproca para Teorema da Convergência Dominada de Lebesgue. Este teorema é útil para o controle em passagem ao limite em termos não lineares das integrais em questão.

Teorema 1.4.5. Seja $\left(f_{n}\right)$ uma sequência de funções em $L^{p}(X, \mu)$ e seja $f \in L^{p}(X, \mu)$ tal que $\left\|f_{n}-f\right\|_{L^{p}} \rightarrow 0$.

Então, existe uma subsequência $\left(f_{n_{k}}\right)$ e uma função $h \in L^{p}(X, \mu)$ tal que:

(a) $f_{n_{k}}(x) \rightarrow f(x)$ q.t.p. em $X$,

(b) $\left|f_{n_{k}}(x)\right| \leq h(x) \quad \forall k$, q.t.p. em $X$. 
Demonstração. Ver [4], pág. 94, Teorema 4.9.

Outro modo de convergência que será muito útil no decorrer desse trabalho é a convergência em medida.

Definição 1.4.6. Dizemos que uma sequência de funções $\left(f_{n}\right)$ converge em medida para uma função $f$ se:

$$
\lim _{n \rightarrow \infty} \mu\left(\left\{x \in X:\left|f_{n}(x)-f(x)\right| \geq \varepsilon\right\}\right)=0,
$$

para todo $\varepsilon>0$.

Observação 1.4.7. Observe que convergência em $L^{p}$ implica em convergência em medida. De fato, considere $E_{n}(\varepsilon)=\left\{x \in X:\left|f_{n}(x)-f(x)\right| \geq \varepsilon\right\}$, então:

$$
\int_{X}\left|f_{n}-f\right|^{p} d \mu \geq \int_{E_{n}(\varepsilon)}\left|f_{n}-f\right|^{p} d \mu \geq \varepsilon^{p} \mu\left(E_{n}(\varepsilon)\right) .
$$

Como $\left\|f_{n}-f\right\|_{L^{p}} \rightarrow 0$ e $\varepsilon>0$ segue que $\mu\left(E_{n}(\varepsilon)\right) \rightarrow 0$, quando $n \rightarrow \infty$.

Vale observar que a recíproca é falsa. O contra-exemplo é dado pela seguinte sequência de funções:

$$
f_{n}=n^{-1 / p} \mathcal{X}_{[0, n]}
$$

Consideramos $X=\mathbb{R}$ e $\mu$ como sendo medida de Lebesgue. Note que $f_{n} \rightarrow 0$ em medida, mas $f_{n} \nrightarrow 0$ em $L^{p}(\mathbb{R})$.

Observamos também que convergência pontual não implica em convergência em medida, exceto se $X$ tem medida finita (veja Teorema 7.12 de [1]). Consideramos mais uma vez $X=\mathbb{R}$, $\mu$ como a medida de Lebesgue e a sequência de funções $f_{n}=\mathcal{X}_{[n, n+1]}$. É fácil ver que $f_{n}$ converge a 0 para todo ponto, mas não em medida. Por outro lado, a proposição seguinte nos mostra que sempre podemos extrair uma subsequência que converge q.t.p. de uma sequência que converge em medida.

Proposição 1.4.8. Seja $\left(f_{n}\right)$ uma sequência de funções mensuráveis definidas em $X$. Se $f_{n} \rightarrow f$ em medida, então existe uma subsequência $\left(f_{n_{j}}\right)$ de $\left(f_{n}\right)$ tal que $f_{n_{j}}(x) \rightarrow f(x)$ q.t.p em $X$.

Demonstração. Considere $\left(f_{n}\right)$ tal que $f_{n} \rightarrow f$ em medida. 
Dado $\varepsilon=\frac{1}{2^{k}}, k \in \mathbb{N}$, existe $n_{k}$ tal que

$$
\mu\left(\left\{x \in X:\left|f_{n}(x)-f(x)\right| \geq \frac{1}{2^{k}}\right\}\right), \quad \forall n \geq n_{k} \leq \varepsilon .
$$

Definimos $X_{k}=\left\{x \in X:\left|f_{n}(x)-f(x)\right| \geq \frac{1}{2^{k}}\right\}$. Para $N \in \mathbb{N}$ fixado, seja $A_{N}=\bigcup_{k \geq N} X_{k}$. Então:

$$
\mu\left(A_{N}\right) \leq \sum_{k \geq N} \mu\left(X_{k}\right) \leq \sum_{k \geq N} \frac{1}{2^{k}}=\frac{1}{2^{N-1}}
$$

Se $x \in X \backslash A_{N}$, então:

$$
x \in A_{N}^{c}=\bigcap_{k \geq N} X_{k}^{c}=\bigcap_{k \geq N}\left\{x \in X:\left|f_{n}(x)-f(x)\right|<\frac{1}{2^{k}}\right\},
$$

ou seja, $\left|f_{n_{k}}(x)-f(x)\right|<\frac{1}{2^{k}}$, para todo $k \geq N$. Portanto, para algum $N \in \mathbb{N}$, temos:

$$
f_{n_{k}}(x) \rightarrow f(x) \quad \text { sobre } A_{N}^{c}
$$

Observando que $\mu\left(A_{1}\right) \geq 1$ e $A_{1} \supset A_{2} \supset \ldots \supset A_{N}$, tem-se:

$$
0 \leq \mu(A)=\mu\left(\bigcap_{N \geq 1} A_{N}\right)=\lim _{N \rightarrow \infty} \mu\left(A_{N}\right) \leq \lim _{N \rightarrow \infty} \frac{1}{2^{N-1}}=0 .
$$

Como $f_{n}(x) \rightarrow f(x)$ sobre $X \backslash A_{N}$, para cada $N$, então $f_{n_{k}}(x) \rightarrow f(x)$ sobre $X \backslash A$ e $\mu(A)=0$. Assim,

$$
f_{n_{k}}(x) \rightarrow f(x) \quad \text { q.t.p. } X \text {. }
$$

Apresentamos agora o famoso Teorema da Convergência de Vitali. Tal teorema nos será útil no final do Capítulo 3.

Teorema 1.4.9 (Convergência de Vitali). Seja $\left(f_{n}\right)$ uma sequência de funções mensuráveis em $X$ tal que $\left(f_{n}\right) \subset L^{p}(X, \mu)$, para $1 \leq p<\infty$. Então, $\left(f_{n}\right)$ converge em $L^{p}(X, \mu)$ para $f$ e $f \in L^{p}(X, \mu)$ se, e somente se, $\left(f_{n}\right)$ satisfaz as seguintes propriedades:

(i) $\left(f_{n}\right)$ converge em medida para $f$;

(ii) Para cada $\varepsilon>0$, existe $\delta(\varepsilon)>0$, tal que se $A \in \mathcal{M}$ com $\mu(A)<\delta(\varepsilon)$, então:

$$
\int_{A}\left|f_{n}\right|^{p} d \mu<\varepsilon^{p}, \quad \text { para todo } n \in \mathbb{N} \text {. }
$$

e existe $C \in \mathcal{M}$ com $\mu(C)<\infty$ tal que

$$
\int_{X \backslash C}\left|f_{n}\right|^{p} d \mu<\varepsilon^{p}, \quad \text { para todo } n \in \mathbb{N} \text {; }
$$


Demonstração. $(\Rightarrow)$ Assumimos que $f_{n}, f \in L^{p}(X, \mu)$ e $\left\|f_{n}-f\right\|_{L^{p}} \rightarrow 0$. Como $\left(f_{n}\right)$ converge para $f$ em $L^{p}(X, \mu)$, temos que $\left(f_{n}\right)$ converge para $f$ em medida. Pela desigualdade de Minkowski, temos:

$$
\left(\int_{X}\left|f_{n}\right|^{p} d \mu\right)^{\frac{1}{p}}=\left(\int_{X}\left|f_{n}-f+f\right|^{p} d \mu\right)^{\frac{1}{p}} \leq\left(\int_{X}|f|^{p} d \mu\right)^{\frac{1}{p}}+\left(\int_{X}\left|f_{n}-f\right|^{p} d \mu\right)^{\frac{1}{p}} .
$$

Dado $\varepsilon>0$, definimos $F_{\lambda}=\left\{x \in E:|f(x)|^{p} \geq \lambda>0\right\}$. Note que $F_{\lambda}$ tem medida finita, pois

$$
\lambda \mu\left(F_{\lambda}\right) \leq \int_{F_{\lambda}}|f|^{p} d \mu \leq \int_{E}|f|^{p} d \mu \leq M
$$

observe que na terceira desigualdade acima usamos que $f \in L^{p}(X, \mu)$, por hipótese, e assim, $\lambda \mu\left(F_{\lambda}\right) \leq M$.

Fazendo $\lambda=\frac{1}{n}$, temos:

$$
\lim _{n \rightarrow \infty} \int_{F_{\frac{1}{n}}}|f|^{p} d \mu=\lim _{n \rightarrow \infty} \int_{X}|f|^{p} \mathcal{X}_{F_{\frac{1}{n}}} d \mu
$$

Como $|f|^{p} \mathcal{X}_{F_{\frac{1}{n}}} \longrightarrow|f|^{p}$ q.t.p e $|f|^{p} \mathcal{X}_{F_{\frac{1}{n}}} \leq|f|^{p}$, onde $|f|^{p} \in L^{1}(X, \mu)$, segue pela Proposição 1.4.4, pág. 24, que:

$$
\lim _{n \rightarrow \infty} \int_{F_{\frac{1}{n}}}|f|^{p} d \mu=\lim _{n \rightarrow \infty} \int_{X}|f|^{p} \mathcal{X}_{F_{\frac{1}{n}}} d \mu=\int_{X}|f|^{p} d \mu .
$$

Agora, como $f \in L^{p}$, existe $\delta_{0}>0$ tal que para cada $A$ e $B \in \mathcal{M}$ tais que $\mu(A)<\delta_{0}$ e $\mu(B)<\infty$, temos:

$$
\int_{X \backslash B}|f|^{p} d \mu<\left(\frac{\varepsilon}{4}\right)^{p} \text { e } \int_{A}|f|^{p} d \mu<\left(\frac{\varepsilon}{4}\right)^{p} .
$$

Além disso, existe $n_{0} \in \mathbb{N}$ tal que se $n \geq n_{0}$, então:

$$
\left\|f_{n}-f\right\|_{L^{p}}<\left(\frac{\varepsilon}{4}\right)^{p} .
$$

Por (1.3), se $n \geq n_{0}$ e $\mu(A)<\delta_{0}$, temos:

$$
\left(\int_{X \backslash B}\left|f_{n}\right|^{p} d \mu\right)^{\frac{1}{p}} \leq\left(\int_{X \backslash B}|f|^{p} d \mu\right)^{\frac{1}{p}}+\left(\int_{X \backslash B}\left|f_{n}-f\right|^{p} d \mu\right)^{\frac{1}{p}}<\frac{\varepsilon}{4}+\frac{\varepsilon}{4}=\frac{\varepsilon}{2}
$$

e

$$
\left(\int_{A}\left|f_{n}\right|^{p} d \mu\right)^{\frac{1}{p}} \leq\left(\int_{A}|f|^{p} d \mu\right)^{\frac{1}{p}}+\left(\int_{A}\left|f_{n}-f\right|^{p} d \mu\right)^{\frac{1}{p}}<\frac{\varepsilon}{4}+\frac{\varepsilon}{4}=\frac{\varepsilon}{2} .
$$

Logo

$$
\int_{X \backslash B}\left|f_{n}\right|^{p} d \mu<\varepsilon^{p} \quad \text { e } \quad \int_{A}\left|f_{n}\right|^{p} d \mu<\varepsilon^{p} .
$$


Sejam $B_{1}, B_{2}, \ldots, B_{n_{0}}$ conjuntos mensuráveis de medidas finitas e $\delta_{1}, \delta_{2}, \ldots, \delta_{n_{0}}$ tais que:

$$
\begin{aligned}
& \int_{X \backslash B_{i}}\left|f_{i}\right|^{p} d \mu<\varepsilon^{p} \quad \text { se } 1 \leq i \leq n_{0} \\
& \int_{A}\left|f_{i}\right|^{p} d \mu<\varepsilon^{p} \quad \text { se } 1 \leq i \leq n_{0} \text { e } \mu(A)<\delta_{i} .
\end{aligned}
$$

Tomando $C=B \cup B_{1} \cup B_{2} \cup \ldots \cup B_{n_{0}}$ e $\delta=\min \left\{\delta_{0}, \delta_{1}, \delta_{2}, \ldots, \delta_{n_{0}}\right\}$, então:

$$
\int_{X \backslash C}\left|f_{n}\right|^{p} d \mu<\varepsilon^{p} \quad \forall n \geq 1 \text { e } \mu(C)<\infty
$$

e

$$
\int_{A}\left|f_{n}\right|^{p} d \mu<\varepsilon^{p} \quad \text { se } \mu(A)<\delta \forall n \geq 1
$$

$(\Leftarrow)$ Agora assumimos que $\left(f_{n}\right)$ converge em medida para $f$. Pela Proposição 1.4.8, existe uma subsequência $\left(f_{n_{k}}\right)$ de $\left(f_{n}\right)$, tal que $f_{n_{k}}(x) \rightarrow f(x)$ q.t.p. Pela condição (ii), existe $C$ e pelo Lema de Fatou para a subsequência $\left(f_{n_{k}}\right)$, temos:

$$
\int_{X \backslash C}|f|^{p} d \mu=\int_{X \backslash C} \liminf _{k \rightarrow \infty}\left|f_{n_{k}}\right|^{p} d \mu \leq \liminf _{k \rightarrow \infty} \int_{X \backslash C}\left|f_{n_{k}}\right|^{p} d \mu<\varepsilon^{p},
$$

onde $\mu(C)<\infty$, e além disso, ainda pela condição (ii), temos que:

$$
\int_{A}|f|^{p} d \mu=\int_{A} \liminf _{k \rightarrow \infty}\left|f_{n_{k}}\right|^{p} d \mu \leq \liminf _{k \rightarrow \infty} \int_{A}\left|f_{n_{k}}\right|^{p} d \mu<\varepsilon^{p}
$$

para todo $A \in \mathcal{M}$ tal que $\mu(A)<\delta$.

Seja $E_{n}=\left\{x \in E:\left|f_{n}(x)-f(x)\right|>\frac{\varepsilon}{\mu(C)}\right\} \bigcap C$. Como $f_{n}$ converge em medida para $f$, então existe $n_{0} \in \mathbb{N}$ tal que $\mu\left(E_{n}\right)<\delta$, para todo $n \geq n_{0}$. Note que $E_{n} \subset C \subset X$, assim:

$$
\begin{aligned}
\int_{X}\left|f_{n}-f\right|^{p} d \mu & =\int_{X \backslash C}\left|f_{n}-f\right|^{p} d \mu+\int_{C \backslash E_{n}}\left|f_{n}-f\right|^{p} d \mu+\int_{E_{n}}\left|f_{n}-f\right|^{p} d \mu \\
& \leq \int_{X \backslash C}\left|f_{n}\right|^{p} d \mu+\int_{X \backslash C}|f|^{p} d \mu+\int_{C \backslash E_{n}}\left|f_{n}-f\right|^{p} d \mu+\int_{E_{n}}\left|f_{n}\right|^{p} d \mu+\int_{E_{n}}|f|^{p} d \mu \\
& \leq 5 \varepsilon .
\end{aligned}
$$

Portanto, $\left\|f_{n}-f\right\|_{L^{p}} \rightarrow 0$ e $f \in L^{p}(X, \mu)$. 


\section{Capítulo 2}

\section{Existência de Solução para dados em}<smiles>[AlH][AlH2]</smiles>

Este capítulo é baseado em Ciarlet [7] e Leray-Lions [13]. Apresentaremos neste capítulo resultados, hoje em dia clássicos, que são essenciais para a investigação de diversas Equações Diferenciais Parciais Quasilineares e, em particular, o problema

$$
\left\{\begin{array}{rlll}
-\Delta_{p} u & =f & \text { em } & \Omega \\
u=0 & \text { sobre } & \partial \Omega .
\end{array}\right.
$$

Estabeleceremos os rudimentos das ditas "Técnicas de Monotonia" na Seção 2.1, para então investigarmos o problema (2.1) e seus análogos na Seção 2.2.

Apesar de clássicos, os resultados aqui apresentados não são vistos em geral nos cursos introdutórios sobre Equações Diferenciais Parciais e, por isto, decidimos por destacá-los do capítulo de preliminares.

Ressaltamos, por fim, que as Seções 2.1 e 2.2 são, em sua maior parte, releituras dos trabalhos de Ciarlet [7] e Leray-Lions [13]. Naturalmente, adaptamos os originais para o contexto desta dissertação.

\subsection{Operadores Monótonos}

Entre os operadores não lineares, destacamos a classe dos operadores monótonos que são de extrema importância, pois constituem-se uma ferramenta muito eficaz para estabelecermos a existência de soluções para certos problemas de contorno não-lineares. 
Sejam $X$ um espaço de Banach e $X^{\prime}$ o seu dual. Vamos denotar por $\|\cdot\|$ a norma em $X$, $\|\cdot\|_{*}$ a norma em $X^{\prime}$ e por $(\cdot, \cdot)$ a dualidade entre o espaço de Banach $X$ e seu dual $X^{\prime}$, ou seja,

$$
(f, u)=f(u), \quad \text { para todo } u \in X, f \in X^{\prime} .
$$

Definição 2.1.1. Um operador A, de um espaço de Banach $X$ em um espaço de Banach $Y$, é dito limitado se leva conjuntos limitados de $X$ em conjuntos limitados de $Y$. Em outras palavras, existe $M>0$ tal que:

$$
\|A u\|_{Y} \leq M, \quad \text { para todo } u \in X
$$

Definição 2.1.2. Um operador $A: X \rightarrow X^{\prime}$ é dito monótono se:

$$
(A(u)-A(v), u-v) \geq 0, \quad \text { para todo } u, v \in X
$$

Vamos agora introduzir uma noção de continuidade que aparecerá nas hipóteses dos próximos resultados, sendo essencial para o teorema que garante a sobrejetividade de operadores monótonos.

Definição 2.1.3. Um operador $A: X \rightarrow X^{\prime}$ é dito hemicontínuo se, para quaisquer $u, v, w \in$ $X$, existir $t_{0}=t_{0}(u, v, w)>0$ tal que a função:

$$
\begin{aligned}
f:\left(-t_{0}, t_{0}\right) & \longrightarrow \mathbb{R} \\
t & \longmapsto(A(u+t v), w)
\end{aligned}
$$

é contínua em $t=0$.

O lema a seguir é uma versão do conhecido Truque de Minty. Com ele, podemos compensar através de monotonia a conhecida dificuldade entre se conciliar convergências fracas e operadores não lineares.

Lema 2.1.4. Sejam $X$ um espaço de Banach e $A: X \longrightarrow X^{\prime}$ um operador monótono hemicontínuo. Se $\left(u_{n}\right) \subset X$ for tal que:

$$
u_{n} \rightarrow u \text { em } X, A\left(u_{n}\right) \rightarrow b \text { em } X^{\prime} \text { e } \limsup _{n \rightarrow \infty}\left(A\left(u_{n}\right), u_{n}\right) \leq(b, u)
$$

então

$$
A(u)=b \text {. }
$$


Demonstração. Seja $\left(u_{n}\right)$ como no enunciado. Observe que, como $A$ é monótono, dado $w \in X$, temos:

$$
\left(A\left(u_{n}\right)-A(w), u_{n}-w\right) \geq 0, \quad \forall n \in \mathbb{N} .
$$

Agora, por hipótese:

$$
\limsup _{n \rightarrow \infty}\left(A\left(u_{n}\right), u_{n}\right) \leq(b, u)
$$

e

$$
\limsup _{n \rightarrow \infty}\left(A\left(u_{n}\right), w\right)=(b, w) .
$$

Então, por (2.2)

$$
(b-A(w), v-w) \geq \limsup _{n \rightarrow \infty}\left(A\left(u_{n}\right)-A(w), u_{n}-w\right) \geq 0 .
$$

Agora, sejam $v \in X, t>0$ e defina:

$$
w=u+t v
$$

Segue de (2.3) que

$$
t(b-A(u+t v),-v)=(b-A(w), u-w) \geq 0, \quad \forall t>0 .
$$

Isto é,

$$
(b-A(u+t v), v) \leq 0, \quad \forall t>0 .
$$

Pela hemicontinuidade do operador $A$, segue que:

$$
(b-A(u), v)=\lim _{t \rightarrow 0^{+}}(b-A(u+t v), v), \quad \forall v \in X .
$$

Em particular,

$$
(b-A(u), v) \leq 0, \quad \forall v \in X
$$

Agora, tomando $-v$ em (2.4), segue que:

$$
(b-A(u), v)=0, \quad \forall v \in X,
$$

portanto:

$$
A(u)=b .
$$


O próximo lema é uma versão do resultado principal desta seção para espaços vetoriais normados de dimensão finita.

Lema 2.1.5. Seja $X$ um espaço vetorial normado de dimensão finita e $A: X \longrightarrow X$ um operador limitado e contínuo. Suponha que A seja coercivo no sentido de que:

$$
\lim _{\|v\| \rightarrow \infty} \frac{(A(v), v)}{\|v\|}=\infty
$$

Então temos que A é sobrejetivo.

Demonstração. Primeiramente, observe que todo espaço de dimensão finita é isometricamente isomorfo a $\mathbb{R}^{N}$. De fato, considere que $\operatorname{dim} X=N$ e seja $\left\{x_{1}, \ldots, x_{N}\right\}$ uma base ortonormal para $X$. Definimos então:

$$
\begin{aligned}
T: X & \longrightarrow \mathbb{R}^{N} \\
x & \longmapsto T(x)=\sum_{i=1}^{N} a_{i} e_{i},
\end{aligned}
$$

onde $x=\sum_{i=1}^{N} a_{i} x_{i}$ e $\left\{e_{1}, \ldots, e_{N}\right\}$ representa a base canônica de $\mathbb{R}^{N}$. Observe que $T$ é uma bijeção linear.

Então considerando $\|y\|_{\mathbb{R}^{N}}=\|x\|_{X}$, onde $y=T x$, obtemos que $X$ e $\mathbb{R}^{N}$ são isometricamente isomorfos. Vale lembrar que todas as normas em $\mathbb{R}^{N}$ são equivalentes.

Tendo em vista esse fato, sejam $w \in X$ e $B_{k}=\{v \in X:\|v\| \leq k\}$ com fronteira $S_{k}=\{v \in$ $X:\|v\|=k\}$. Tomando o mesmo $k$ tal que $\|w\|<k$, então pela hipótese do operador $A$ ser coercivo, temos que para qualquer $k$, podemos escolher $R$ suficientemente grande tal que:

$$
(A(v), v) \geq R k \quad \forall v \in S_{R}
$$

Agora, consideremos

$$
\left\{\begin{array}{l}
H: B_{k} \times[0,1] \longrightarrow X \\
H(v, \theta)=\theta A(v)+(1-\theta) \frac{k}{R} v
\end{array}\right.
$$

de modo que $H(v, 0)=\frac{v k}{R}$ e $H(v, 1)=A(v)$.

Note que $H$ é contínuo. Além disso, para $v \in S_{R}$, temos:

$$
\left|\left(\theta A(v)+(1-\theta) \frac{k}{R} v, v\right)\right| \geq k R .
$$

Assim

$$
\|H(v, \theta)\| \geq k, \quad \forall v \in S_{R}
$$


Em particular, $w \notin H\left(S_{R} \times[0,1]\right)$ e então $d\left(H(\cdot, \theta), B_{R}, w\right)$, onde $d(\cdot)$ denota o grau de Brouwer, está bem definido para $\theta \in[0,1]$. Porém, pelo Teorema 1.3.2, pág. 23, temos:

$$
1=d\left(I d \frac{k}{R}, B_{R}, w\right)=d\left(H(\cdot, 0), B_{R}, w\right)=d\left(H(\cdot, 1), B_{R}, w\right)=d\left(A, B_{R}, w\right) .
$$

Portando, como $d\left(A, B_{R}, w\right) \neq 0$, pelo Teorema 1.3.1, pág. 22, existe $v \in B_{R}(0)$ tal que $A(v)=w$.

O próximo teorema é o resultado principal desta seção. As hipóteses sobre o operador $A$ foram encontradas em [13], pág. 98, Teorema 1.

Teorema 2.1.6. Sejam $X$ um espaço de Banach separável e reflexivo e $A: X \longrightarrow X^{\prime}$ um operador limitado e hemicontínuo. Suponha que A é coercivo no sentido de que:

$$
\lim _{\|v\| \rightarrow \infty} \frac{(A(v), v)}{\|v\|}=\infty .
$$

Se A é monótono, então A é sobrejetivo.

Demonstração. Para demonstrar o teorema, com o Lema 2.1.5 estabelecido, usaremos o famoso método de Galerkin para construirmos soluções aproximadas em subespaços de $X$ que tenham dimensão finita e depois usaremos o processo limite. Para maior conveniência do leitor, dividimos a demonstração em passos.

\section{Passo 1: soluções aproximadas}

Como $X$ é separável, existe uma família enumerável linearmente independente $\left\{w_{i}\right\}_{i=1}^{\infty}$ de vetores $w_{i} \in X$ tal que $\bigcup_{n=1}^{\infty} X_{n}$ é denso em $X$, onde $X_{n}=\operatorname{span}(w)_{i=1}^{n}$.

Afirmação 2.1.1. Se $f \in X^{\prime}$, existe $u_{n} \in X_{n}$ tal que:

$$
\left(A\left(u_{n}\right), w\right)=(f, w), \quad \forall w \in X_{n}
$$

De fato, se $\theta_{1}, \theta_{2}, \ldots, \theta_{n} \in X^{\prime} \operatorname{com}\left(\theta_{i}, w_{j}\right)=\delta_{j}^{i}$, definimos $P_{n}$ por:

$$
P_{n} v^{\prime}=\sum_{j=1}^{n}\left(v^{\prime}, w_{j}\right) \theta_{j}
$$

Denotando $B_{n}(v)=P_{n}(A(v))$ temos que a Afirmação 2.1.1 é equivalente a:

$$
B_{n}\left(u_{n}\right)=P_{n} f \quad \text { em } \quad X_{n}^{\prime}
$$


Tomamos $X_{n}^{\prime}$ o espaço gerado por $\theta_{1}, \theta_{2}, \ldots, \theta_{n}$, então $B_{n}$ aplica $X_{n}$ em $X_{n}^{\prime}$. Como $\left(B_{n}(v), v\right)=$ $(A(v), v)$, a condição de coercividade é satisfeita e a afirmação segue aplicando o resultado quando estamos no caso de dimensão finita.

\section{Passo 2: processo limite}

Pela Afirmação 2.1.1, temos:

$$
\left|\left(A\left(u_{n}\right), u_{n}\right)\right|=\left|\left(f, u_{n}\right)\right| \leq\|f\|_{*}\left\|u_{n}\right\| .
$$

Pela hipótese do operador $A$ ser coercivo, segue que a sequência $u_{n}$ é uniformemente limitada, ou seja, existe $C>0$ tal que:

$$
\left\|u_{n}\right\| \leq C
$$

De fato, se $u_{n}$ não fosse limitada, então teríamos:

$$
\lim _{\left\|u_{n}\right\| \rightarrow \infty}=\frac{\left|\left(A\left(u_{n}\right), u_{n}\right)\right|}{\left\|u_{n}\right\|}=\infty
$$

o que é um absurdo com (2.5).

Agora, como também por hipótese $A$ é um operador limitado, segue que $\left\|A\left(u_{n}\right)\right\|_{*} \leq C$. Sendo $X$ reflexivo, temos também que $X^{\prime}$ é reflexivo. Assim, existe uma subsequência $\left(u_{m}\right)$ de $\left(u_{n}\right)$ tal que:

$$
\left\{\begin{array}{llll}
u_{m} & \rightarrow & \text { em } X \\
A\left(u_{m}\right) & \rightarrow & g & \text { em } X^{\prime}
\end{array}\right.
$$

Afirmação 2.1.2. $g=f e A\left(u_{m}\right) \rightarrow f e m X^{\prime}$.

Pela Afirmação 2.1.1, para $k \geq 1$, temos:

$$
\left(A\left(u_{m}\right), w_{k}\right)=\left(f, w_{k}\right) \quad \forall m \geq k .
$$

Portanto:

$$
\left(g, w_{k}\right)=\lim _{m \rightarrow \infty}\left(A\left(u_{m}\right), w_{k}\right)=\left(f, w_{k}\right)
$$

Assim, para todo $k \geq 1$, temos:

$$
(g, w)=(f, w), \quad w \in \bigcup_{m=1}^{\infty} X_{m} .
$$

Por construção $\cup_{m=1}^{\infty} X_{m}=\cup_{n=1}^{\infty} X_{n}$ é denso em $X$, e assim, segue que $g=f$. Por conseguinte, $A\left(u_{m}\right) \rightarrow f$. 
Agora note que estabelecida a Afirmação 2.1.2, segue direto que:

$$
\lim _{m \rightarrow \infty}\left(A\left(u_{m}\right), u_{m}\right)=\lim _{m \rightarrow \infty}\left(f, u_{m}\right)=(f, u) .
$$

Assim

$$
\left\{\begin{array}{l}
u_{m} \rightarrow u \text { em } X \\
A\left(u_{m}\right) \rightarrow f \text { em } X^{\prime} \\
\left(A\left(u_{m}\right), u_{m}\right) \longrightarrow(f, u),
\end{array}\right.
$$

segue do Lema 2.1.4 que

$$
A(u)=f
$$

Observamos que na versão original deste resultado de Leray e Lions [13], a hipótese do operador $A$ ser hemicontínuo é substituída por uma outra mais forte. Mais precisamente, em [13], pede-se que $A$ seja fracamente estrela contínuo em subespaços de dimensão finita.

\subsection{Existência de Solução para o p-Laplaciano}

Nesta seção, $\Omega \subset \mathbb{R}^{N}$ será um aberto, limitado e conexo. Seja $u \in W_{0}^{1, p}(\Omega)$, onde $1<p<\infty$. O operador $p$-Laplaciano

$$
-\Delta_{p} u=-\operatorname{div}\left(|\nabla u|^{p-2} \nabla u\right)
$$

age em $W_{0}^{p}(\Omega)$ tomando valores em $W^{-1, p^{\prime}}(\Omega)$ da seguinte maneira:

$$
\left(-\Delta_{p} u, v\right)=\int_{\Omega}|\nabla u|^{p-2} \nabla u \cdot \nabla v, \quad \text { para } u, v \in W_{0}^{1, p}(\Omega) .
$$

Observação 2.2.1. Observe que se $u \in W_{0}^{1, p}(\Omega)$, então $-\Delta_{p} u \in W^{-1, p^{\prime}}(\Omega)$. De fato, seja $u \in W_{0}^{1, p}(\Omega)$ e note que $\left(-\Delta_{p} u, v\right)$ é linear em $v$. Além disso, para toda $v \in W_{0}^{1, p}(\Omega)$ temos, por Cauchy-Schwarz, que:

$$
\left|\left(-\Delta_{p} u, v\right)\right|=\left.\left.\left|\int_{\Omega}\right| \nabla u\right|^{p-2} \nabla u \cdot \nabla v\left|\leq \int_{\Omega}\right| \nabla u\right|^{p-1}|\nabla v| .
$$

e, usando a desigualdade de Hölder:

$$
\left|\left(-\Delta_{p} u, v\right)\right| \leq \int_{\Omega}|\nabla u|^{p-1}|\nabla v| \leq\left(\int_{\Omega}|\nabla u|^{p}\right)^{\frac{1}{p^{\prime}}}\left(\int_{\Omega}|\nabla v|^{p}\right)^{\frac{1}{p}}=\left\|\left.u\right|_{W_{0}^{1, p}} ^{p-1}\right\| v \|_{W_{0}^{1, p} .}
$$


Lema 2.2.2. O operador p-Laplaciano $-\Delta_{p}$ é limitado.

Demonstração. Seja $u \in W_{0}^{1, p}(\Omega)$ tal que $\|u\|_{W_{0}^{1, p}} \leq M$, onde $M>0$. Temos:

$$
\begin{aligned}
\left\|-\Delta_{p} u\right\|_{W^{-1, p^{\prime}}} & =\sup _{\|v\| \leq 1}\left|\left(-\Delta_{p} u, v\right)\right| \\
& \leq \sup _{\|v\| \leq 1}\left(\int_{\Omega}|\nabla u|^{p}\right)^{\frac{1}{p^{\prime}}}\left(\int_{\Omega}|\nabla v|^{p}\right)^{\frac{1}{p}} \\
& \leq\|u\|_{W_{0}^{1, p}}^{p-1} \leq M^{p-1} .
\end{aligned}
$$

Portanto, $-\Delta_{p}$ é limitado.

Para o que segue, queremos mostrar que o operador $p$-Laplaciano é monótono, ou seja,

$$
\left(-\Delta_{p} u+\Delta_{p} v, u-v\right) \geq 0, \quad \forall u, v \in W_{0}^{1, p}(\Omega)
$$

Para tanto, será necessário introduzir o seguinte lema.

Lema 2.2.3. Sejam $x, y \in \mathbb{R}^{N}$ e $p \geq 2$. Então:

$$
\left(|x|^{p-2}-|y|^{p-2}\right)\left(|x|^{2}-|y|^{2}\right)+\left(|x|^{p-2}+|y|^{p-2}\right)|x-y|^{2}=2\left(|x|^{p-2} x-|y|^{p-2} y\right) \cdot(x-y) .
$$

Demonstração. A demonstração deste lema sai por um cálculo direto.

$$
\begin{aligned}
& \left(|x|^{2}-|y|^{2}\right)\left(|x|^{p-2}-|y|^{p-2}\right)+|x-y|^{2}\left(|x|^{p-2}+|y|^{p-2}\right) \\
& =(x-y) \cdot\left[(x+y)\left(|x|^{p-2}-|y|^{p-2}\right)+2(x-y) \cdot\left(|x|^{p-2}+|y|^{p-2}\right)\right] \\
& =2(x-y) \cdot\left(|x|^{p-2} x-|y|^{p-2} y\right) .
\end{aligned}
$$

Proposição 2.2.4. O operador p-Laplaciano é monótono.

Demonstração. Devemos mostrar que:

$$
\left(-\Delta_{p} u+\Delta_{p} v, u-v\right)=\int_{\Omega}\left(|\nabla u|^{p-2} \nabla u-|\nabla v|^{p-2} \nabla v\right) \cdot(\nabla u-\nabla v) \geq 0 .
$$

De fato, sejam $x, y \in \mathbb{R}^{N}$. Vamos dividir em dois casos, quando $p \geq 2$ e $1<p<2$.

Caso 1: $p \geq 2$

Como $p \geq 2$, temos a seguinte desigualdade:

$$
|x-y|^{p}=|x-y|^{p-2}|x-y|^{2} \leq 2^{p-3}|x-y|^{2}\left(|x|^{p-2}+|y|^{p-2}\right) .
$$


Combinando a desigualdade acima com o Lema 2.2.3, e notando que $x \mapsto|x|^{p-2}$ e $x \mapsto|x|^{2}$ são monótonas não-decrescentes, temos:

$$
|x-y|^{p} \leq 2^{p-2}\left(|x|^{p-2} x-|y|^{p-2} y\right)(x-y) .
$$

Assim, para o caso $p \geq 2$ temos:

$$
\begin{aligned}
\left(-\Delta_{p} u+\Delta_{p} v, u-v\right) & =\int_{\Omega}\left(|\nabla u|^{p-2} \nabla u-|\nabla v|^{p-2} \nabla v\right) \cdot(\nabla u-\nabla v) \\
& \geq \frac{1}{2^{p-2}} \int_{\Omega}|\nabla u-\nabla v|^{p} \geq 0 \quad \forall u, v \in W_{0}^{1, p}(\Omega) .
\end{aligned}
$$

Caso 2: $1<p<2$

Para $1<p<2$, temos a desigualdade:

$$
(p-1)|x-y|^{2}(1+|x|+|y|)^{p-2} \leq\left(|x|^{p-2} x-|y|^{p-2} y\right)(x-y) .
$$

De fato, começamos notando que:

$$
\left(|x|^{p-2} x-|y|^{2} y\right) \cdot(x-y)=(x-y) \int_{0}^{1} \frac{d}{d t}\left(|y+t(x-y)|^{p-2}(y+t(x-y))\right) d t .
$$

Agora, calculando a derivada no integrando acima, temos:

$$
\begin{aligned}
\left(|x|^{p-2} x-|y|^{2} y\right) \cdot(x-y) & =|x-y|^{2} \int_{0}^{1}|y+t(x-y)|^{p-2} d t \\
& +(p-2) \int_{0}^{1}|y+t(x-y)|^{p-4}\left((y+t(x-y)) \cdot(x-y)^{2} d t .\right.
\end{aligned}
$$

Por Cauchy-Schwarz, segue que:

$$
((y+t(x-y)) \cdot(x-y))^{2} \leq|y+t(x-y)|^{2}|x-y|^{2} .
$$

Como $1<p<2$, segue que $(p-2)<0$ e a desigualdade acima se reverte quando multiplicamos por $(p-2)$ em ambos os lados. Portanto:

$$
\begin{aligned}
\left(|x|^{p-2} x-|y|^{2} y\right) \cdot(x-y) & =|x-y|^{2} \int_{0}^{1}|y+t(x-y)|^{p-2} d t \\
& +(p-2) \int_{0}^{1}|y+t(x-y)|^{p-4}\left((y+t(x-y)) \cdot(x-y)^{2} d t .\right. \\
& \geq(p-1)|x-y|^{2} \int_{0}^{1}|y+t(x-y)|^{p-2} d t \\
& \geq(p-1)|x-y|^{2}(1+|y|+|x|)^{p-2} .
\end{aligned}
$$


Logo:

$$
\left(|x|^{p-2} x-|y|^{2} y\right) \cdot(x-y) \geq(p-1)|x-y|^{2}(1+|y|+|x|)^{p-2} .
$$

Assim, para o caso $1<p<2$, temos:

$$
\begin{aligned}
\left(-\Delta_{p} u+\Delta_{p} v, u-v\right) & =\int_{\Omega}\left(|\nabla u|^{p-2} \nabla u-|\nabla v|^{p-2} \nabla v\right) \cdot(\nabla u-\nabla v) \\
& \geq(p-1) \int_{\Omega}|\nabla u-\nabla v|^{2}(1+|\nabla u|+|\nabla v|)^{p-2} \geq 0 \quad \forall u, v \in W_{0}^{1, p}(\Omega) .
\end{aligned}
$$

Com isso, concluímos que o operador $-\Delta_{p}$ é monótono para $1<p<\infty$.

Com estes resultados em mãos, podemos provar o seguinte resultado, essencial no decorrer do texto.

Teorema 2.2.5. Sejam $\Omega \subset \mathbb{R}^{N}$ um aberto, limitado, conexo e $1<p<\infty$.

(a) O operador $-\Delta_{p}$ é hemicontínuo e coercivo.

(b) Para cada $f \in W^{-1, p^{\prime}}(\Omega)$, o problema:

$$
\left\{\begin{aligned}
-\Delta_{p} u=f & \text { em } \Omega \\
u=0 & \text { sobre } \partial \Omega
\end{aligned}\right.
$$

tem uma solução única $u \in W_{0}^{1, p}(\Omega)$.

Demonstração. (a) Para provarmos que o operador $-\Delta_{p}$ é hemicontínuo, basta observarmos que a aplicação:

$$
t \longmapsto\left(-\Delta_{p}(u+t v), \varphi\right)
$$

é contínua em $t=0$, para toda $\varphi \in W_{0}^{1, p}(\Omega)$. De fato, seja $\left(t_{n}\right)$ uma sequência tal que $t_{n} \longrightarrow 0$. Então:

$$
\begin{aligned}
& u_{n}=u+t_{n} v \quad \longrightarrow u \mathrm{em} W_{0}^{1, p}(\Omega) \\
& \nabla u_{n}=\nabla u+t_{n} \nabla v \longrightarrow \nabla u \operatorname{em~} L^{p}(\Omega) .
\end{aligned}
$$

Assim, pelo Teorema 1.4.5, pág. 24, passando a uma subsequência se necessário, temos:

$$
\nabla u_{n} \longrightarrow \nabla u \quad \text { q.t.p em } \Omega
$$

e existe $g \in L^{p}(\Omega)$ tal que:

$$
\left|\nabla u_{n}\right| \leq g \in L^{p}(\Omega)
$$


Tome $\varphi \in W_{0}^{1, p}(\Omega)$. Afirmamos que:

$$
\int_{\Omega}\left|\nabla u_{n}\right|^{p-2} \nabla u_{n} \cdot \nabla \varphi \longrightarrow \int_{\Omega}|\nabla u|^{p-2} \nabla u \cdot \nabla \varphi
$$

De fato, basta ver que:

$$
\left|\nabla u_{n}\right|^{p-2} \nabla u_{n} \cdot \nabla \varphi \longrightarrow|\nabla u|^{p-2} \nabla u \cdot \nabla \varphi
$$

e

$$
\left|\nabla u_{n}\right|^{p-2}\left|\nabla u_{n} \cdot \nabla \varphi\right| \leq\left|\nabla u_{n}\right|^{p-1}|| \nabla \varphi\left|\leq g^{p-1}\right| \nabla \varphi \mid \in L^{1}(\Omega) .
$$

Então pelo Teorema da Convergência Dominada de Lebesgue, segue a afirmação. Portanto, temos que o operador $p$-Laplaciano $-\Delta_{p}$ é hemicontínuo.

Agora, para provarmos que $-\Delta_{p} u$ é coercivo, note que:

$$
\frac{\left(-\Delta_{p} u, u\right)}{\|u\|_{W_{0}^{1, p}}}=\frac{\int_{\Omega}|\nabla u|^{p}}{\|u\|_{W_{0}^{1, p}}}=\|u\|_{W_{0}^{1, p}}^{p-1}
$$

e quando $\|u\|_{W_{0}^{1, p}} \rightarrow \infty$ temos que:

$$
\frac{\left(-\Delta_{p} u, u\right)}{\|u\|_{W_{0}^{1, p}}} \rightarrow \infty
$$

Assim o operador $-\Delta_{p}$ é coercivo.

(b) Pela Proposição 2.2.4 o operador $-\Delta_{p}$ é monótono, logo, pelo item (a), satisfaz todas as hipóteses do Teorema 2.1.6, e assim temos a existência da $u$.

Agora, para a unicidade, suponha que:

$$
\left\{\begin{array}{rll}
-\Delta_{p} u_{i}= & \text { em } \Omega \\
u_{i}= & 0 & \text { sobre } \partial \Omega
\end{array}\right.
$$

para $i=1,2$. Suponha também, sem perda de generalidade, que ao menos uma $u_{i}$ não seja identicamente nula.

Então, em particular:

$$
\begin{array}{rll}
-\Delta_{p} u_{1}+\Delta_{p} u_{2}=0 & \text { em } \Omega \\
u_{1}-u_{2}=0 & \text { sobre } \partial \Omega .
\end{array}
$$

Assim, para toda $\varphi \in W_{0}^{1, p}(\Omega)$, segue que:

$$
\int_{\Omega}\left(\left|\nabla u_{1}\right|^{p-2} \nabla u_{1}-\left|\nabla u_{2}\right|^{p-2} \nabla u_{2}\right) \cdot \nabla \varphi=0
$$


Tome $\varphi=u_{1}-u_{2}$. Logo

$$
\int_{\Omega}\left(\left|\nabla u_{1}\right|^{p-2} \nabla u_{1}-\left|\nabla u_{2}\right|^{p-2} \nabla u_{2}\right) \cdot\left(\nabla u_{1}-\nabla u_{2}\right)=0
$$

Analisamos dois casos:

Caso 1: $p \geq 2$

Como $p \geq 2$, segue que

$$
\int_{\Omega}\left|\nabla u_{1}-\nabla u_{2}\right|^{p} \leq 2^{p-2} \int_{\Omega}\left(\left|\nabla u_{1}\right|^{p-2} \nabla u_{1}-\left|\nabla u_{2}\right|^{p-2} \nabla u_{2}\right) \cdot\left(\nabla u_{1}-\nabla u_{2}\right)=0 .
$$

Assim, $\nabla\left(u_{1}-u_{2}\right)=0$ q.t.p em $\Omega$.

Como $u_{1}-u_{2} \in W_{0}^{1, p}(\Omega)$, segue pela desigualdade de Poincaré que:

$$
\left\|u_{1}-u_{2}\right\|_{L^{p}} \leq K\left\|\nabla u_{1}-\nabla u_{2}\right\|_{L^{p}}=0 .
$$

Isto é,

$$
u_{1}=u_{2} \quad \text { q.t.p. em } \Omega \text {. }
$$

Caso 2: $1<p<2$

Como $1<p<2$, temos:

$$
\int_{\Omega} \frac{\left|\nabla u_{1}-\nabla u_{2}\right|^{2}}{\left(1+\left|\nabla u_{1}+\right| \nabla u_{2} \mid\right)^{2-p}} \leq \frac{1}{p-1} \int_{\Omega}\left(\left|\nabla u_{1}\right|^{p-2} \nabla u_{1}-\left|\nabla u_{2}\right|^{p-2} \nabla u_{2}\right) \cdot\left(\nabla u_{1}-\nabla u_{2}\right)=0 .
$$

Logo, $\nabla\left(u_{1}-u_{2}\right)=0$ q.t.p em $\Omega$. Portanto, de maneira análoga ao caso anterior, temos:

$$
u_{1}=u_{2} \quad \text { q.t.p em } \Omega \text {. }
$$

O resultado abaixo generaliza o Teorema 2.2.5.

Teorema 2.2.6. Seja $f \in W^{-1, p^{\prime}}(\Omega)$. Então existe uma única $u \in W_{0}^{1, p}(\Omega)$ tal que:

$$
(P 1)\left\{\begin{array}{cccc}
-\Delta_{p} u+h(x, u) & = & f & \text { em } \Omega \\
u & = & 0 & \text { sobre } \partial \Omega
\end{array}\right.
$$

onde $h$ é Carathéodory e existe $k_{1}>0$ tal que $|h(x, t)| \leq k_{1}$ para todo $x \in \Omega$ e $t \in \mathbb{R}$. 
Demonstração. Considere o seguinte problema: Dada $u_{n-1} \in W_{0}^{1, p}(\Omega)$, encontrar $u_{n} \in W_{0}^{1, p}(\Omega)$ tal que:

$$
(P 2)\left\{\begin{array}{cccc}
-\Delta_{p} u_{n}+h\left(x, u_{n-1}\right) & = & f & \text { em } \Omega \\
u_{n} & = & 0 & \text { sobe } \partial \Omega .
\end{array}\right.
$$

Observe que $h\left(x, u_{n-1}\right) \in W^{-1, p^{\prime}}(\Omega)$, para todo $n \in \mathbb{N}$, pois $h \in L^{\infty}(\Omega)$. Além disso, veja que pelo Teorema 2.2 .5 o problema $(P 2)$ está bem posto.

Tomando $u_{n}$ como função teste em $(P 2)$, obtemos que:

$$
\begin{aligned}
\int_{\Omega}\left|\nabla u_{n}\right|^{p} & =\left(f, u_{n}\right)-\int_{\Omega} h\left(x, u_{n-1}\right) u_{n} \\
& \leq\|f\|_{W^{-1, p^{\prime}}}\left\|u_{n}\right\|_{W_{0}^{1, p}}+k_{1}\left\|u_{n}\right\|_{L^{1}} \\
& \leq\|f\|_{W^{-1, p^{\prime}}}\left\|u_{n}\right\|_{W_{0}^{1, p}}+k_{1} k_{2}\left\|u_{n}\right\|_{W_{0}^{1, p}}
\end{aligned}
$$

onde $k_{2}$ é a constante da desigualdade de Poincaré.

Agora, tomando $c=\max \left\{1, k_{1} k_{2}\right\}$, temos que dado $\varepsilon>0$ :

$$
\begin{aligned}
\left\|u_{n}\right\|_{W_{0}^{1, p}} & \leq c\left(\|f\|_{W^{-1, p^{\prime}}}+1\right)\left\|u_{n}\right\|_{W_{0}^{1, p}} \\
& =\frac{c\left(\|f\|_{W^{-1, p^{\prime}}}+1\right)}{\varepsilon^{\frac{1}{p}}}\left\|u_{n}\right\|_{W_{0}^{1, p}} \varepsilon^{\frac{1}{p}} \\
& \leq\left[\frac{c\left(\|f\|_{W^{-1, p^{\prime}}}+1\right)}{\varepsilon^{\frac{1}{p}}}\right]^{p^{\prime}} \frac{1}{p^{\prime}}+\frac{\left\|u_{n}\right\|_{W_{0}^{1, p}} \varepsilon}{p} .
\end{aligned}
$$

A última desigualdade segue por Young. Então:

$$
\left(1-\frac{\varepsilon}{p}\right)\left\|u_{n}\right\|_{W_{0}^{1, p}}^{p} \leq K_{\varepsilon, p}
$$

onde $K_{\varepsilon, p}=\left[\frac{c\left(\|f\|_{\left.W^{-1, p^{\prime}}+1\right)}\right.}{\varepsilon^{\frac{1}{p}} p}\right]^{p^{\prime}}$.

Porém, fazendo $\varepsilon=\frac{p}{2}$, segue que:

$$
\left\|u_{n}\right\|_{W_{0}^{1, p}}^{p} \leq 2 K_{\varepsilon, p}, \quad \forall n \in \mathbb{N}
$$

Assim, como pelo Teorema 1.2.9, pag. $22, W_{0}^{1, p}(\Omega)$ está imerso compactamente em $L^{q}(\Omega)$, 
para todo $1 \leq q<\frac{N p}{N-p}$, existe $u \in W_{0}^{1, p}(\Omega)$ tal que, a menos de subsequências, temos:

$$
\begin{array}{ll}
u_{n} \rightarrow u & \text { em } W_{0}^{1, q}(\Omega) \\
u_{n} \rightarrow u & \text { em } L^{q}(\Omega) \\
u_{n} \rightarrow u & \text { q.t.p. em } \Omega .
\end{array}
$$

Agora, observamos que dado $v \in W_{0}^{1, p}(\Omega)$ tal que $\|v\|_{W_{0}^{1, p}} \leq 1$, aplicando a desigualdade de Hölder, temos:

$$
\begin{aligned}
\left(-\Delta_{p} u_{n}, v\right)=\int_{\Omega}\left|\nabla u_{n}\right|^{p-2} \nabla u_{n} \cdot \nabla v & \leq \int_{\Omega}\left|\nabla u_{n}\right|^{p-1}|\nabla v| \\
& \leq\left\|\left.\nabla u_{n}\right|_{L^{p}} ^{p-1}|| \nabla v\right\|_{L^{p}} \\
& \leq \|\left. u_{n}\right|_{W_{0}^{1, p}} ^{p-1} \\
& \leq 2 K_{\varepsilon, p}^{p^{\prime}} \quad \forall n \in \mathbb{N} .
\end{aligned}
$$

Sendo assim,

$$
\left\|-\Delta_{p} u_{n}\right\|_{W^{-1, p^{\prime}}} \leq 2 K_{\varepsilon, p}^{p^{\prime}}
$$

e pela reflexibilidade de $W^{-1, p^{\prime}}(\Omega)$, existe $b \in W^{-1, p^{\prime}}$ tal que, a menos de subsequências,

$$
-\Delta_{p} u_{n} \rightarrow b \quad \text { em } W^{-1, p^{\prime}}(\Omega)
$$

Precisamos mostrar que $b=-\Delta_{p} u$. Observe que:

$$
\left(-\Delta_{p} u_{n}, u_{n}\right)=\int_{\Omega} f u_{n}-h\left(x, u_{n-1}\right) u_{n}
$$

Por (2.6), segue que:

$$
\lim _{n \rightarrow \infty} \int_{\Omega} f u_{n}=\int_{\Omega} f u
$$

Afirmação 2.2.1.

$$
\lim _{n \rightarrow \infty} \int_{\Omega} h\left(x, u_{n-1}\right) u_{n}=\int_{\Omega} h(x, u) u .
$$

De fato, como $h$ é Carathéodory, temos:

$$
\lim _{n \rightarrow \infty} h\left(x, u_{n-1}\right) u_{n}=h(x, u) u \quad \text { q.t.p em } \Omega \text {. }
$$

Além disso, pela recíproca do Teorema da Convergência Dominada de Lebesgue, pág. 22, existe $g \in L^{p}(\Omega)$ tal que, a menos de subsequências,

$$
\left|u_{n}\right| \leq g \quad \text { q.t.p em } \Omega
$$


Logo, por hipótese, segue que:

$$
\left|h\left(x, u_{n-1}\right) u_{n}\right| \leq C g \in L^{p}(\Omega) .
$$

Então, pelo Teorema da Convergência Dominada de Lebesgue, temos que:

$$
\lim _{n \rightarrow \infty} \int_{\Omega} h\left(x, u_{n-1}\right) u_{n}=\int_{\Omega} h(x, u) u .
$$

Assim, a Afirmação 2.2.1 está provada.

Agora, veja que por (2.8) e (2.9), segue que:

$$
\lim _{n \rightarrow \infty}\left(-\Delta_{p} u_{n}, u_{n}\right)=\int_{\Omega} f u-h(x, u) u .
$$

Entretanto, por outro lado, notemos que:

$$
-\Delta_{p} u_{n}=f-h\left(x, u_{n-1}\right) \quad \text { em } W^{-1, p^{\prime}}(\Omega)
$$

Porém, observamos que:

$$
h\left(x, u_{n-1}\right) \longrightarrow h(x, u) \quad \text { em } L^{p^{\prime}}(\Omega) .
$$

De fato, basta observar que $h\left(x, u_{n-1}\right) \rightarrow h(x, u)$ q.t.p. em $\Omega$ e que $\left|h\left(x, u_{n-1}\right)\right| \leq C \in L^{p^{\prime}}(\Omega)$ q.t.p. em $\Omega$, por hipótese.

Logo, pelo Teorema da Convergência Dominada de Lesbesgue para $L^{p^{\prime}}$, pág. 24, segue que:

$$
h\left(x, u_{n-1}\right) \longrightarrow h(x, u) \quad \text { em } L^{p^{\prime}}(\Omega),
$$

como queríamos.

Em particular, como:

$$
L^{p^{\prime}}(\Omega) \hookrightarrow W^{-1, p^{\prime}}(\Omega)
$$

temos que:

$$
h\left(x, u_{n-1}\right) \longrightarrow h(x, u) \quad \text { em } W^{-1, p^{\prime}}(\Omega) .
$$

Assim, por (2.7), (2.10) e (2.11), segue que:

$$
b=f-h(x, u) .
$$

Agora, usando o "Truque de Minty", combinando (2.10) e (2.12), obtemos que:

$$
\lim _{n \rightarrow \infty}\left(-\Delta_{p} u_{n}, u_{n}\right)=(b, u) .
$$


Porém, pelo Lema 2.1.4, (2.6), (2.7) e (2.8), temos que:

$$
b=-\Delta_{p} u
$$

Portanto, por (2.7), (2.10), (2.11) e (2.14):

$$
-\Delta_{p} u+h(x, u)=f \quad \text { em } W^{-1, p^{\prime}}(\Omega)
$$

e o resultado segue. 


\section{Capítulo 3}

\section{Existência de Solução para dados em} $M(\Omega)$

Neste capítulo, estudaremos os principais tópicos proposto no presente trabalho. Estes resultados são versões adaptadas do trabalho de Boccardo \& Gallouet em [2].

Na Seção 3.1, estudaremos a existência de solução do problema $(P)$ com dados em espaços de medida. Na Seção 3.2, estudaremos a existência de solução do problema $(P)$ com termo forçante linear, mas com uma certa regularidade. Em seguida, na Seção 3.3, estaremos interessados em estudar a existência de solução do problema p-Laplaciano com termo forçante não linear de ordem zero.

Em todo este capítulo, $\Omega \subset \mathbb{R}^{N}$ é um aberto, limitado e conexo.

\subsection{Existência de Solução para o p-Laplaciano}

Consideremos, para uma $f$ arbitrária em $M(\Omega)$, a equação:

$$
(P) \quad\left\{\begin{array}{rlll}
-\Delta_{p} u & = & f & \text { em } \Omega \\
u= & 0 & \text { em } & \partial \Omega .
\end{array}\right.
$$

Por simplicidade, nesta seção dada $f \in M(\Omega)$, denotaremos:

$$
\int_{\Omega} \varphi f:=\int_{\Omega} \varphi d f
$$

Antes de prosseguir, precisaremos introduzir um novo conceito de solução fraca. 
Definição 3.1.1. Uma função u é dita solução fraca de $(P)$ se satisfaz:

$$
\left\{\begin{array}{l}
u \in W_{0}^{1,1}(\Omega),|\nabla u|^{p-2} \nabla u \in L_{l o c}^{1}(\Omega) e \\
\int_{\Omega}|\nabla u|^{p-2} \nabla u \cdot \nabla \varphi=\int_{\Omega} f \varphi, \text { para toda } \varphi \in C_{c}^{\infty}(\Omega) .
\end{array}\right.
$$

Pelo Teorema 2.2.5, pág. 38, quando $f \in W^{-1, p^{\prime}}(\Omega)$, sabemos que $(P)$ tem uma única solução fraca $u$ tal que:

$$
\begin{gathered}
u \in W_{0}^{1, p}(\Omega) \\
\int_{\Omega}|\nabla u|^{p-2} \nabla u \cdot \nabla v=\int_{\Omega} f v, \text { para toda } v \in W_{0}^{1, p}(\Omega) .
\end{gathered}
$$

Em geral, quando $f \in M(\Omega)$ ou $L^{1}(\Omega)$, não temos que $|\nabla u|^{p-1} \in L^{p^{\prime}}(\Omega)$. Isto poderia comprometer o termo:

$$
\int_{\Omega}|\nabla u|^{p-2} \nabla u \cdot \nabla v
$$

quando $v \in W_{0}^{1, p}(\Omega)$, isto é, $\nabla v \in L^{p}(\Omega)$. Isto torna necessária a inclusão da hipótese $|\nabla u|^{p-2} \nabla u \in L_{l o c}^{1}(\Omega)$ na definição acima e a alteração do espaço das funções teste de $W_{0}^{1, p}(\Omega)$ $\operatorname{para} C_{c}^{\infty}(\Omega)$.

Nossa estratégia será a de investigar uma versão preliminar de $(P)$, ligeiramente mais regular, onde:

$$
f \in W^{-1, p^{\prime}}(\Omega) \cap L^{1}(\Omega)
$$

Observe que, neste caso, o melhor que poderíamos esperar seria que:

$$
u \in W_{0}^{1, p-1}(\Omega)
$$

De fato, supondo que $f=\operatorname{div}(g)$, com $g \in L^{1}(\Omega)$, teríamos:

$$
-\operatorname{div}\left(|\nabla u|^{p-2} \nabla u\right)=\operatorname{div}(g)
$$

o que primeiramente, nos forneceria que:

$$
|\nabla u|^{p-1}=g \in L^{1}(\Omega)
$$

de modo que $u \in W_{0}^{1, p-1}(\Omega)$.

Na realidade, obteremos estimativas em $W_{0}^{1, q}(\Omega)$ onde $1 \leq q<\frac{N}{N-1}(p-1)$. Vale observar que $\frac{N}{N-1}(p-1)>(p-1)$. 
Além disso, se $p \in(2-1 / N, N]$, então $1<(N /(N-1))(p-1)$. De fato, como $2-1 / N<p$, temos que:

$$
((N-1+N) / N)<p
$$

A desigualdade acima nos dá que $N+N-p N<1$, e portanto, $1<(N /(N-1))(p-1)$. Com isso, nossa estimativa abrangerá uma classe maior de regularidade sobre $u$.

Lema 3.1.2. Para todo $1 \leq q<(N /(N-1))(p-1)$ e $B>0$, existe $C>0$, dependendo de $q, p, \Omega$ e $B$, tal que se $f \in W^{-1, p^{\prime}}(\Omega) \cap L^{1}(\Omega)$ e u é a solução de (3.1), então $\|u\|_{W_{0}^{1, q}} \leq C$, sempre que $\|f\|_{L^{1}(\Omega)} \leq B$.

Demonstração. Tome $f \in W^{-1, p^{\prime}}(\Omega) \cap L^{1}(\Omega)$ tal que $\|f\|_{L^{1}(\Omega)} \leq B$.

Por simplicidade de notação, usaremos o símbolo $c_{i}$ para denotar uma constante arbitrária,

$$
c_{i}=c_{i}(p, q, B, \Omega)>0
$$

Consideremos então, $n \in \mathbb{N}$ e $\psi$ dada por:

$$
\psi(s)=\left\{\begin{array}{lll}
n & \text { se } & s>n \\
s & \text { se } \quad-n \leq s \leq n \\
-n & \text { se } \quad s<-n
\end{array}\right.
$$

Pelo Teorema 2.2.5, pág 38, existe $u \in W_{0}^{1, p}(\Omega)$ tal que:

$$
\int_{\Omega}|\nabla u|^{p-2} \nabla u \cdot \nabla \varphi=\int_{\Omega} f \varphi \quad \forall \varphi \in W_{0}^{1, p}(\Omega) .
$$

Observe que $\psi(u) \in W_{0}^{1, p}(\Omega)$, pois $\psi$ é localmente Lipschitz (para mais detalhes, veja Gilbarg \& Trudinger em [14], Teorema 7.8, pág.153).

Neste caso, tomando $\varphi=\psi(u)$ em (3.3), temos que:

$$
\int_{\Omega} \psi^{\prime}(u)|\nabla u|^{p-2} \nabla u \cdot \nabla u=\int_{\Omega} f \psi(u) .
$$

Consideremos então o conjunto:

$$
D_{n}=\{x \in \Omega:|u(x)| \leq n\} .
$$

Como $\psi^{\prime}(u)=1$ em $D_{n}$, segue de (3.4) que: 


$$
\int_{D_{n}}|\nabla u|^{p}=\int_{D_{n}} f \psi(u)=\int_{D_{n}} f u \leq n\|f\|_{L^{1}}=n B .
$$

Por outro lado, consideremos outra função teste para obter estimativas melhores. De fato, consideremos:

$$
\psi(s)=\left\{\begin{array}{cll}
1 & \text { se } & s>n+1 \\
s-n & \text { se } & n \leq s \leq n+1 \\
0 & \text { se } & -n \leq s \leq n \\
s+n & \text { se } & -n-1 \leq s \leq-n \\
-1 & \text { se } & s<-n-1 .
\end{array}\right.
$$

Seja também o conjunto:

$$
B_{n}=\{x \in \Omega: n \leq|u(x)| \leq n+1\} .
$$

Afirmação 3.1.1. Para cada $n \in \mathbb{N}$

$$
\int_{B_{n}}|\nabla u|^{p} \leq\|f\|_{L^{1}}
$$

Para provar (3.9), basta observar que:

$$
\left\{\begin{array}{l}
\psi^{\prime}(u)=1 \\
|\psi(u)|=|u(x)-n|
\end{array} \quad \text { q.t.p. em } B_{n} .\right.
$$

Além disso, como $n \leq|u(x)| \leq n+1$, então:

$$
|u(x)-n| \leq 1
$$

Logo, como $\psi(u) \in W_{0}^{1, p}(\Omega)$ (ver novamente em [14], Teorema 7.8, pág.153) tomando-se $\varphi=$ $\psi(u)$ em (3.3), segue que:

$$
\int_{B_{n}}|\nabla u|^{p}=\int_{B_{n}} f \psi(u)
$$

Assim, como:

$$
\int_{B_{n}}|f| \psi(u)=\int_{B_{n}}|f||u-n| \leq \int_{B_{n}}|f|,
$$

combinando-se (3.10) e (3.11), obtemos que:

$$
\int_{B_{n}}|\nabla u|^{p} \leq\|f\|_{L^{1}} \leq B
$$

o que prova a Afirmação 3.1.1. 
Agora, vamos combinar (3.6) e (3.9) para obtermos estimativas em todo $\Omega$.

De fato, primeiramente observamos que, pela desigualdade de Hölder para $1 \leq q<p$, temos:

$$
\int_{B_{n}}|\nabla u|^{q} \leq\left(\int_{B_{n}}|\nabla u|^{p}\right)^{q / p}\left(\operatorname{med}\left(B_{n}\right)\right)^{(p-q) / p} \leq B^{q / p} \operatorname{med}(\Omega)^{\frac{p-q}{q}},
$$

onde $\operatorname{med}\left(B_{n}\right)$ e med $(\Omega)$ denotam a medida de Lebesgue dos conjuntos $B_{n}$ e $\Omega$, respectivamente.

Além disso, observamos que como $1 \leq q<p \leq N$, então $q^{*}<p^{*}$ e por Imersão de Sobolev,

$$
W_{0}^{1, p}(\Omega) \hookrightarrow L^{q^{*}}(\Omega)
$$

de modo que $u \in L^{q^{*}}(\Omega)$ e, em particular,

$$
\int_{\Omega}|u|^{q^{*}}<\infty
$$

Temos ainda, pela definição de $B_{n}$ :

$$
\int_{B_{n}}\left|u_{n}\right|^{q^{*}} \geq \int_{B_{n}} n^{q^{*}}=n^{q^{*}} \operatorname{med}\left(B_{n}\right)
$$

e assim

$$
\operatorname{med}\left(B_{n}\right) \leq \frac{1}{n^{q^{*}}} \int_{B_{n}}|u|^{q^{*}} .
$$

Além disso, por (3.12) e (3.14), para $c_{1}=B^{q / p}$ :

$$
\int_{B_{n}}|\nabla u|^{q} \leq c_{1}\left(\int_{B_{n}}|u|^{q^{*}}\right)^{(p-q) / p} \frac{1}{n^{q^{*}((p-q) / p)}}
$$

Assim, dado $n_{0} \in \mathbb{N}$, tomando o somatório:

$$
\sum_{n=n_{0}}^{\infty} \int_{B_{n}}|\nabla u|^{q} \leq c_{1} \sum_{n=n_{0}}^{\infty}\left(\int_{B_{n}}|u|^{q^{*}}\right)^{(p-q) / p} \frac{1}{n^{q^{*}((p-q) / p)}}
$$

Então, aplicando a desigualdade de Hölder para séries, com os expoentes $\frac{p}{p-q}$ e $\frac{p}{q}$, obtemos que:

$$
\sum_{n=n_{0}}^{\infty} \int_{B_{n}}|\nabla u|^{q} \leq c_{1}\left(\sum_{n=n_{0}}^{\infty} \int_{B_{n}}|u|^{q^{*}}\right)^{(p-q) / p} \sum_{n=n_{0}}^{\infty}\left(\frac{1}{n^{q^{*}((p-q) / q)}}\right)^{q / p} .
$$

Note que $\bigcup_{n=0}^{\infty} B_{n}=\Omega$, então pela subaditividade da medida de Lebesgue:

$$
\sum_{n=n_{0}}^{\infty} \int_{B_{n}}|u|^{q^{*}}<\infty
$$


Além disso, por (3.17) e por (3.6), temos que:

$$
\int_{\Omega}|\nabla u|^{q} \leq n_{0}^{q / p} c_{2}+c_{1}\|u\|_{L^{q *}}^{q *(p-q) / p}\left(\sum_{n=n_{0}}^{\infty} \frac{1}{n^{q *((p-q) / q)}}\right)^{q / p}
$$

onde $c_{2}=c_{1}(\operatorname{med}(\Omega))^{(p-q) / p}$.

De fato, observe que $\Omega=D_{n_{0}} \cup\left(\cup_{n=n_{0}}^{\infty}\right) B_{n}$, onde $D_{n_{0}}=\left\{x \in \Omega:|u(x)| \leq n_{0}\right\}$ e $B_{n_{0}}=\{x \in$ $\left.\Omega: n_{0} \leq|u(x)| \leq n_{0}+1\right\}$. Assim,

$$
\int_{\Omega}|\nabla u|^{q} \leq \int_{D_{n_{o}}}|\nabla u|^{q}+\sum_{n=n_{0}}^{\infty} \int_{B_{n_{0}}}|\nabla u|^{q} .
$$

Na primeira integral do lado direito, pela desigualdade de Hölder com expoentes $p / q$, $(p-$ q)/q e por (3.6) segue que:

$$
\int_{D_{n_{o}}}|\nabla u|^{q} \leq\left(\int_{D_{n_{o}}}|\nabla u|^{p}\right)^{q / p}\left(\operatorname{med}\left(D_{n_{0}}\right)\right)^{(p-q) / p} \leq n_{0}^{q / p} c_{1}(\operatorname{med}(\Omega))^{(p-q) / p}=n_{0}^{q / p} c_{2} .
$$

Na última, usamos que $\sum_{n=n_{0}}^{\infty} \int_{B_{n}}|u|^{q^{*}} \leq \int_{\Omega}|u|^{q^{*}}$, e assim:

$$
\left(\sum_{n=n_{0}}^{\infty} \int_{B_{n}}|u|^{q^{*}}\right)^{(p-q) / p} \leq\|u\|_{L^{q^{*}(\Omega)}}^{q^{*}(p-q) / p}
$$

Logo, a estimativa (3.18) está provada. Seja $\widehat{c}=\max \left\{c_{1}, c_{2}\right\}$, então:

$$
\begin{aligned}
\int_{\Omega}|\nabla u|^{q} & \leq \widehat{c}\left(1+n_{0}^{q / p}+\|u\|_{L^{q^{*}}}^{q^{*}(p-q) / p}\left(\sum_{n=n_{0}}^{\infty} \frac{1}{n^{q^{*}((p-q) / q)}}\right)^{q / p}\right) \\
& =\widehat{c}\left(1+\frac{1}{n_{0}^{q / p}}\right) n_{0}^{q / p}+\widehat{c}\|u\|_{L^{q^{*}}}^{q^{*}(p-q) / p}\left(\sum_{n=n_{0}}^{\infty} \frac{1}{n^{q^{*}((p-q) / q)}}\right)^{q / p} \\
& \leq \widehat{c}\left(1+\frac{1}{n_{0}^{q / p}}\right)\left(n_{0}^{q / p}+\|u\|_{L^{q^{*}}}^{q^{*}(p-q) / p}\left(\sum_{n=n_{0}}^{\infty} \frac{1}{n^{q^{*}((p-q) / q)}}\right)^{q / p}\right)
\end{aligned}
$$

Pelo Teorema 1.2.8, pág. 22, obtemos que:

$$
\|u\|_{L^{q^{*}}}^{q} \leq c_{3}\left(n_{0}^{q / p}+\|u\|_{L^{q^{*}}}^{q^{*}(p-q) / p}\left(\sum_{n=n_{0}}^{\infty} \frac{1}{n^{q^{*}((p-q) / q)}}\right)^{q / p}\right),
$$

onde $c_{3}=K \widehat{c}\left(1+\frac{1}{n_{0}^{q / p}}\right)$, sendo $K$ a constante do Teorema da Imersão de Sololev.

Lembrando que $2-1 / N<p \leq N$. Devemos analisar dois casos.

Caso 1: $p=N$

Neste caso, temos:

$$
q^{*}(p-q) / p=\frac{N q(N-q)}{(N-q) N}=q
$$




$$
q^{*}((p-q) / q)=\frac{N q(N-q)}{(N-q) q}=N \geq 2 .
$$

Como $q^{*}((p-q) / q) \geq 2$, então $\left(\sum_{n=n_{0}}^{\infty} \frac{1}{n^{q^{*}((p-q) / q)}}\right)^{q / p}$ converge e se escolhermos um $n_{0}$ suficientemente grande temos que $\lim _{n_{0} \rightarrow \infty} \sum_{n=n_{0}}^{\infty} \frac{1}{n^{q^{*}((p-q) / q)}} \longrightarrow 0$. Assim, escolhendo um $n_{0}$ em (3.20) tal que $\left(\sum_{n=n_{0}}^{\infty} \frac{1}{n^{q^{*}((p-q) / q)}}\right)^{q / p}<\frac{1}{2^{q / p} c_{3}}$ temos que

$$
\|u\|_{L^{q^{*}}} \leq c_{4}
$$

onde $c_{4}=\left(\frac{c_{3} n_{0}^{q / p} 2^{q / p}}{2^{q / p}-1}\right)^{1 / q}$.

Então, por (3.18),

$$
\|\nabla u\|_{L^{q}} \leq c_{5}
$$

o que prova o lema para o caso $p$ igual a $N$.

Caso 2: $p<N$

Se $p$ é estritamente menor que $N$, então $q^{*}((p-q) / p)=(q N /(N-q)) /((p-q) / p)<q$ e $q^{*}((p-q) / q)=(q N /(N-q)) /((p-q) / q)=N(p-q) /(N-q)>1$. De fato, como por hipótese $q<(N /(N-1))(p-1) \Rightarrow(N-1) q<N(p-1) \Rightarrow 0<N p-N-q(N-1) \Rightarrow N<$ $N p+(-N+1) \Rightarrow N-q<N(p-q) \Rightarrow 1<N(p-q) /(N-q)$. Assim, a série $\sum_{n=n_{0}}^{\infty} \frac{1}{n^{q^{*}((p-q) / q)}}$ converge e escolhendo $n_{0}=1 \mathrm{em}(3.20)$, temos:

$$
\|u\|_{L^{q^{*}}} \leq c_{6}
$$

e assim, segue que:

$$
\|\nabla u\|_{L^{q}} \leq c_{7}
$$

o que prova o lema para o caso $p<N$.

Observação 3.1.3. É importante notar que no caso $p=N, q$ é estritamente menor que $(N /(N-1))(p-1)$, o que nos permite aplicar o Teorema da Imersão de Sobolev $(q<N)$. No caso $p<N$, a limitação de q garante a convergência da série do lado direito de (3.18).

Com o Lema 3.1.2, provamos o teorema a seguir.

Teorema 3.1.4. Existe uma solução fraca u para o problema $(P)$ com a regularidade $u \in$ $W_{0}^{1, q}(\Omega)$ para todo $1 \leq q<(N /(N-1))(p-1)$. 
Demonstração. Consideremos $f \in M(\Omega)$.

Combinando o Corolário 1.1.30, pág. 17, e pelo Teorema 1.1.35, pág. 19, existe $\left(f_{n}\right) \subset$ $L^{p^{\prime}}(\Omega) \subset L^{1}(\Omega) \cap W^{-1, p^{\prime}}(\Omega)$ tal que $f_{n} \longrightarrow f$ no sentido das distribuições.

Além disso, pelo Teorema 1.1.26, pág. 15,

$$
\left\|f_{n}\right\|_{L^{1}} \leq B=\|f\|_{M(\Omega)}
$$

Consideramos então o seguinte problema aproximado:

$$
\left\{\begin{aligned}
-\Delta_{p} v=f_{n} & \text { em } \Omega \\
v=0 & \text { sobre } \partial \Omega
\end{aligned}\right.
$$

Pelo Teorema 2.2.5, pág. 38, existe uma única $u_{n} \in W_{0}^{1, p}(\Omega)$ solução de (3.21). Além disso, aplicando-se o Lema 3.1.2, obtemos que

$$
\left\|u_{n}\right\|_{W_{0}^{1, q}} \leq C=C(p, q, \Omega, N)
$$

e $1 \leq q<\frac{N}{N-1}(p-1)$

Usando a reflexibilidade de $W_{0}^{1, q}(\Omega)$ e pelo Teorema 1.2 .9 , pág. 22 , existe $u \in W_{0}^{1, q}(\Omega)$ tal que, a menos de subsequências,

$$
\begin{array}{ll}
u_{n} \rightarrow u & \text { em } W_{0}^{1, q}(\Omega) \\
u_{n} \rightarrow u & \text { em } L^{q}(\Omega) \\
u_{n} \rightarrow u & \text { q.t.p em } \Omega
\end{array}
$$

Falta mostrar que:

$$
-\Delta_{p} u_{n} \longrightarrow-\Delta_{p} u \quad \text { em } W^{-1, p^{\prime}}(\Omega)
$$

No caso em que $q \geq p$, basta repetir o mesmo argumento do Teorema 2.2.6, pág. 40.

Para evitarmos este inconveniente e, ao mesmo tempo, exibir uma abordagem alternativa, iremos seguir Boccardo \& Gallouet, [2], pág. 156, neste ponto.

Para tanto, será necessário a seguinte afirmação.

Afirmação 3.1.2. : $\left(u_{n}\right)$ é relativamente compacta em $W_{0}^{1, q}(\Omega)$ para todo $1 \leq q<(N /(N-$ 1)) $(p-1)$

Faremos a prova deste resultado no final demonstração. 
Supondo que a Afirmação 3.1.2 seja verdadeira, em particular temos que, a menos de subsequências:

$$
\nabla u_{n} \longrightarrow \nabla u \quad \text { em } L^{q}(\Omega), \text { para todo } q \in\left[1, \frac{N}{N-1}(p-1)\right) .
$$

De fato, existe $g \in L^{q}(\Omega)$ tal que, a menos de subsequências,

$$
\nabla u_{n} \longrightarrow g \quad \text { em } L^{q}(\Omega)
$$

Em particular, pela observação logo após ao Teorema 1.1.35, pág. 20, segue que:

$$
\nabla u_{n} \longrightarrow g \quad \text { no sentido das distribuições. }
$$

Agora, por (3.22), temos que:

$$
\nabla u_{n} \rightarrow \nabla u \quad \text { em } L^{q}(\Omega) .
$$

Assim, novamente pelo comentário logo após ao Teorema 1.1.35, pág. 20, segue que:

$$
\nabla u_{n} \longrightarrow \nabla u \quad \text { no sentido das distribuições. }
$$

Combinando (3.24) e (3.25) juntamente com a unicidade da convergência em distribuição, segue que:

$$
g=\nabla u
$$

e assim, vale (3.23).

Agora, por (3.23) e pelo Teorema 1.4.5, pág. 24, temos a menos de subsequências (denotada ainda por $\left.\left(\nabla u_{n}\right)\right)$ que:

$$
\begin{aligned}
& \nabla u_{n}(x) \longrightarrow \nabla u(x) \quad \text { q.t.p. em } \Omega \\
& \left|\nabla u_{n}(x)\right| \leq g(x) \quad \text { para todo } n, \text { q.t.p. em } \Omega,
\end{aligned}
$$

onde $g \in L^{q}(\Omega)$, para todo $q \in[1,(N /(N-1))(p-1))$.

Por (3.26), $\left|\nabla u_{n}\right|^{p-2} \nabla u_{n} \longrightarrow|\nabla u|^{p-2} \nabla u$ q.tp em $\Omega$ e $\left.\left.|| \nabla u_{n}\right|^{p-2} \nabla u_{n}|\leq| \nabla u_{n}\right|^{p-1} \leq g^{p-1}$. Como $g^{p-1} \in L^{r}(\Omega)$, para todo $r \in\left[1, \frac{N}{N-1}\right)$, então $\left|\nabla u_{n}\right|^{p-2} \nabla u_{n} \in L^{r}(\Omega)$, para todo $r \in$ $\left[1, \frac{N}{N-1}\right)$. Assim, pelo Teorema da Convergência Dominada, temos:

$$
\left|\nabla u_{n}\right|^{p-2} \nabla u_{n} \longrightarrow|\nabla u|^{p-2} \nabla u \quad \text { em } L^{r}(\Omega), \text { para todo } r \in\left[1, \frac{N}{N-1}\right) .
$$


Com a convergência obtida em (3.27), segue que:

$$
\lim _{n \rightarrow \infty} \int_{\Omega}\left|\nabla u_{n}\right|^{p-2} \nabla u_{n} \cdot \nabla \varphi=\int_{\Omega}|\nabla u|^{p-2} \nabla u \cdot \nabla \varphi, \quad \forall \varphi \in C_{c}^{\infty}(\Omega) .
$$

Além disso,

$$
|\nabla u|^{p-2} \nabla u \in L^{r}(\Omega) \hookrightarrow L_{l o c}^{1}(\Omega)
$$

Como $f_{n} \longrightarrow f$ vagamente em $M(\Omega)$, então pela Definição 1.1.16, pág. 12, temos:

$$
\lim _{n \rightarrow \infty} \int_{\Omega} f_{n} \varphi=\int_{\Omega} f \varphi
$$

Ainda falta provar a Afirmação 3.1.2. Para tanto, dado $\varepsilon>0$ consideramos $\psi: \mathbb{R} \longrightarrow \mathbb{R}$, onde:

$$
\psi(s)=\left\{\begin{array}{lll}
\varepsilon & \text { se } \quad s>\varepsilon \\
s & \text { se } \quad-\varepsilon \leq s \leq \varepsilon \\
-\varepsilon & \text { se } \quad s<-\varepsilon .
\end{array}\right.
$$

Usando (3.1) com $f=f_{n}$ e $f_{m}, u=u_{n}$ e $u_{m}$ e $v=\psi\left(u_{n}-u_{m}\right)$, obtemos:

$$
\begin{aligned}
\int_{\Omega} \psi^{\prime}\left(u_{n}-u_{m}\right) & \left(\left|\nabla u_{n}\right|^{p-2} \nabla u_{n}-\left|\nabla u_{m}\right|^{p-2} \nabla u_{m}\right)\left(\nabla u_{n}-\nabla u_{m}\right) \\
& =\int_{\Omega}\left(f_{n}-f_{m}\right) \psi\left(u_{n}-u_{m}\right) .
\end{aligned}
$$

Como $\|f\|_{L^{1}(\Omega)} \leq B$, por (3.29) para $p \geq 2$, temos que:

$$
\int_{D_{n, m, \varepsilon}} \frac{1}{2^{2-p}}\left|\nabla u_{n}-\nabla u_{m}\right|^{p} \leq 2 \varepsilon B
$$

e para $2-1 / N<p<2$, temos que:

$$
\int_{D_{n, m, \varepsilon}} \frac{p-1}{\left(1+\left|\nabla u_{n}\right|+\left|\nabla u_{m}\right|\right)^{2-p}}\left|\nabla u_{n}-\nabla u_{m}\right|^{2} \leq 2 \varepsilon B
$$

onde $D_{n, m, \varepsilon}=\left\{x \in \Omega ;\left|u_{n}(x)-u_{m}(x)\right| \leq \varepsilon\right\}$.

Para ver (3.30) e (3.31), basta aplicar a monotonicidade do operador $p$-Laplaciano. Para $p \geq 2$, temos:

$$
\left(\left|\nabla u_{n}\right|^{p-2} \nabla u_{n}-\left|\nabla u_{m}\right|^{p-2} \nabla u_{m}\right) \cdot\left(\nabla u_{n}-\nabla u_{m}\right) \geq \frac{1}{2^{p-2}}\left|\nabla u_{n}-\nabla u_{m}\right|^{p},
$$

e para $(2-1 / N, 2)$ temos:

$$
\left(\left|\nabla u_{n}\right|^{p-2} \nabla u_{n}-\left|\nabla u_{m}\right|^{p-2} \nabla u_{m}\right) \cdot\left(\nabla u_{n}-\nabla u_{m}\right) \geq \frac{(p-1)}{\left(1+\left|\nabla u_{n}\right|+\left|\nabla u_{m}\right|\right)^{2-p}}\left|\nabla u_{n}-\nabla u_{m}\right|^{2} .
$$


Com as desigualdades acima e recordando que em $D_{n, m, \varepsilon}$ temos $\psi^{\prime}\left(u_{n}-u_{m}\right)=1$, segue que

$$
\int_{\Omega}\left(\left|\nabla u_{n}\right|^{p-2} \nabla u_{n}-\left|\nabla u_{m}\right|^{p-2} \nabla u_{m}\right) \cdot\left(\nabla u_{n}-\nabla u_{m}\right) \geq \int_{\Omega} \frac{1}{2^{p-2}}\left|\nabla u_{n}-\nabla u_{m}\right|^{p},
$$

se $p \geq 2$, e

$$
\int_{\Omega}\left(\left|\nabla u_{n}\right|^{p-2} \nabla u_{n}-\left|\nabla u_{m}\right|^{p-2} \nabla u_{m}\right) \cdot\left(\nabla u_{n}-\nabla u_{m}\right) \geq \int_{\Omega} \frac{(p-1)}{\left(1+\left|\nabla u_{n}\right|+\left|\nabla u_{m}\right|\right)^{2-p}}\left|\nabla u_{n}-\nabla u_{m}\right|^{2},
$$

se $2-1 / N<p<2$. Mas:

$$
\begin{aligned}
\int_{\Omega}\left(\left|\nabla u_{n}\right|^{p-2} \nabla u_{n}-\left|\nabla u_{m}\right|^{p-2} \nabla u_{m}\right) \cdot\left(\nabla u_{n}-\nabla u_{m}\right) & =\int_{D_{n, m, \varepsilon}}\left(f_{n}-f_{m}\right)\left(u_{n}-u_{m}\right) \\
& \leq\left|\int_{D_{n, m, \varepsilon}}\left(f_{n}-f_{m}\right)\left(u_{n}-u_{m}\right)\right| \\
& \leq \int_{D_{n, m, \varepsilon}}\left|f_{n}-f_{m}\right|\left|\left(u_{n}-u_{m}\right)\right|
\end{aligned}
$$

Logo:

$$
\begin{aligned}
\int_{\Omega}\left(\left|\nabla u_{n}\right|^{p-2} \nabla u_{n}-\left|\nabla u_{m}\right|^{p-2} \nabla u_{m}\right) \cdot\left(\nabla u_{n}-\nabla u_{m}\right) & \leq \int_{D_{n, m, \varepsilon}}\left|f_{n}-f_{m}\right|\left|\left(u_{n}-u_{m}\right)\right| \\
& \leq \int_{D_{n, m, \varepsilon}}\left|f_{n}-f_{m}\right| \varepsilon \\
& \leq \int_{D_{n, m, \varepsilon}}\left(\left|f_{n}\right|+\left|f_{m}\right|\right) \varepsilon \\
& =\varepsilon\left(\int_{D_{n, m, \varepsilon}}\left|f_{n}\right|+\int_{D_{n, m, \varepsilon}}\left|f_{m}\right|\right) \\
& \leq 2 \varepsilon B .
\end{aligned}
$$

e assim seguem de (3.30) e (3.31).

Para $p \geq 2$ por (3.30) e pela desigualdade de Hölder, temos:

$$
\int_{D_{n, m, \varepsilon}}\left|\nabla u_{n}-\nabla u_{m}\right| \leq \varepsilon^{1 / p} c_{1}\left(\int_{D_{n, m, \varepsilon}}\left(2^{2-p}\right)^{p^{\prime} / p}\right)^{1 / p^{\prime}}
$$

onde $c_{1}=(2 B)^{1 / p}$. Já para o caso $2-1 / N<p<2$ por (3.31) e novamente usando a desigualdade de Hölder, temos:

$$
\int_{D_{n, m, \varepsilon}}\left|\nabla u_{n}-\nabla u_{m}\right| \leq \varepsilon^{1 / 2} c_{2}\left(\int_{D_{n, m, \varepsilon}} \frac{p-1}{\left(1+\left|\nabla u_{n}\right|+\left|\nabla u_{m}\right|\right)^{2-p}}\right)^{1 / 2},
$$

onde $c_{2}=(2 B)^{1 / 2}$.

Notando que ambas as integrais do lado direito das desigualdades acima são finitas, denotamos por $c_{3}$ e $c_{4}$, respectivamente, seus valores. Agora, tomando $c_{5}=\max \left\{c_{1} c_{3}, c_{2} c_{4}\right\}$, junto com a estimativa de $u_{n}$ em $W_{0}^{1, q}(\Omega)$, obtemos: 


$$
\int_{D_{n, m, \varepsilon}}\left|\nabla u_{n}-\nabla u_{m}\right| \leq c_{5} \varepsilon^{1 / s}
$$

onde $s$ depende de $p$. A estimativa (3.32) é usada para provar que $\left(\nabla u_{n}\right)$ é uma sequência de Cauchy em $L^{1}(\Omega)$. Sabemos que:

$$
\int_{\Omega}\left|\nabla\left(u_{n}-u_{m}\right)\right|=\int_{D_{n, m, \varepsilon}}\left|\nabla\left(u_{n}-u_{m}\right)\right|+\int_{\Omega \backslash D_{n, m, \varepsilon}}\left|\nabla\left(u_{n}-u_{m}\right)\right|
$$

Usando a desigualdade de Hölder na segunda parcela da soma do lado direito da igualdade acima com os expoentes conjugados $q$ e $q^{\prime}$, temos:

$$
\begin{aligned}
\int_{\Omega \backslash D_{n, m, \varepsilon}}\left|\nabla\left(u_{n}-u_{m}\right)\right| & \leq\left(\int_{\Omega \backslash D_{n, m, \varepsilon}}\left|\nabla\left(u_{n}-u_{m}\right)\right|^{q}\right)^{1 / q}\left(\int_{\Omega \backslash D_{n, m, \varepsilon}}\right)^{1 / q^{\prime}} \\
& =c_{6} \operatorname{med}\left\{x \in \Omega:\left|u_{n}(x)-u_{m}(x)\right|>\varepsilon\right\}^{1-1 / q},
\end{aligned}
$$

para algum $q \in(1,(N /(N-1))(p-1))$.

Assim, por (3.32) e pela desigualdade acima, temos:

$$
\int_{\Omega}\left|\nabla\left(u_{n}-u_{m}\right)\right| \leq c_{5} \varepsilon^{1 / s}+c_{6} \operatorname{med}\left\{x \in \Omega:\left|u_{n}(x)-u_{m}(x)\right|>\varepsilon\right\}^{1-1 / q}
$$

Como $u_{n}$ converge fortemente para $u$ em $L^{1}(\Omega)$, temos que $u_{n}$ é uma sequência de Cauchy em $L^{1}(\Omega)$, assim $u_{n}$ é uma sequência de Cauchy em medida, ou seja, dado $\varepsilon>0$ existe $n_{0}(\varepsilon) \in \mathbb{N}$ dependendo de $\varepsilon$ tal que para todo $n, m \geq n_{0}(\varepsilon)$ implica $\operatorname{med}\left\{x \in \Omega:\left|u_{n}(x)-u_{m}(x)\right| \geq \varepsilon\right\} \leq \varepsilon$. Logo, (3.33) implica que:

$$
\int_{\Omega}\left|\nabla\left(u_{n}-u_{m}\right)\right| \leq c_{5} \varepsilon^{1 / s}+\varepsilon, \quad \text { para } n, m \geq n_{0}(\varepsilon),
$$

assim provamos que $\left(\nabla u_{n}\right)$ é uma sequência de Cauchy em $L^{1}(\Omega)$. Portanto:

$$
\nabla u_{n} \longrightarrow \nabla u \quad \text { em } L^{1}(\Omega)
$$

Agora vamos mostrar que $\left(\nabla u_{n}\right)$ é uma sequência de Cauchy em $L^{r}(\Omega)$, para todo $1 \leq r \leq$ $q \in[1,(N /(N-1))(p-1))$. De fato, como $\left(u_{n}\right) \in W_{0}^{1, q}$ para todo $q \in[1,(N /(N-1))(p-1))$, então $\left(\nabla u_{n}\right) \in L^{q}(\Omega)$ para todo $q \in[1,(N /(N-1))(p-1))$. Assim, fazendo $r=1-t+t q$ para $t \in[0,1]$ e aplicando a desigualdade de Hölder com os expoentes conjugados $1 / t$ e $1 / 1-t$, obtemos: 


$$
\int_{\Omega}\left|\nabla u_{n}-\nabla u_{m}\right|^{r} \leq\left\|\nabla u_{n}-\nabla u_{m}\right\|_{L^{1}(\Omega)}^{1-t}|| \nabla u_{n}-\nabla u_{m} \|_{L^{q}(\Omega)}^{t},
$$

e portanto $\left(\nabla u_{n}\right)$ é uma sequência de Cauchy em $L^{r}(\Omega)$, pois $\left\|\nabla u_{n}-\nabla u_{m}\right\|_{L^{r}(\Omega)} \leq\left\|\nabla u_{n}\right\|_{L^{r}(\Omega)}+$ $\left\|\nabla u_{m}\right\|_{L^{r}(\Omega)}$ é limitado e $\left(\nabla u_{n}\right)$ é sequência de Cauchy em $L^{1}(\Omega)$.

Logo, $\left(u_{n}\right)$ é uma sequência de Cauchy em $L^{q}(\Omega)$, para todo $q \in\left[1, \frac{N}{N-1}(p-1)\right)$, e portanto, a Afirmação 3.1 .2 é verdadeira.

Como consequência do Teorema 3.1.4, observamos que:

$$
|\nabla u|^{p-2} \nabla u \in L^{r}(\Omega) \quad \text { para cada } r \in\left[1, \frac{N}{N-1}\right) .
$$

De fato, basta observar que $r(p-1) \in\left[1, \frac{N}{N-1}(p-1)\right)$.

Além disso,

$$
\left.\left.\int_{\Omega}|| \nabla u\right|^{p-2} \nabla u\right|^{r} \leq \int_{\Omega}|\nabla u|^{r(p-1)}<\infty
$$

pois $u \in W_{0}^{r(p-1)}(\Omega)$. Ainda, como:

$$
\int_{\Omega}|\nabla u|^{p-2} \nabla u \cdot \nabla \varphi=\int_{\Omega} f \varphi, \quad \forall \varphi \in C_{c}^{\infty}(\Omega),
$$

consideramos $v \in W_{0}^{1, r^{\prime}}(\Omega)$ e $\left(v_{n}\right) \subset C_{c}^{\infty}(\Omega)$ tal que $v_{n} \longrightarrow v$ em $W_{0}^{1, r^{\prime}}(\Omega)$.

No caso em que $r^{\prime}>N, W_{0}^{1, r^{\prime}}(\Omega) \hookrightarrow L^{\infty}(\Omega)$. Logo,

$$
v_{n} \longrightarrow v \quad \operatorname{em~} L^{\infty}(\Omega)
$$

e então

$$
\lim _{n \rightarrow \infty}\left|\int_{\Omega} v_{n}-v d f\right| \leq \lim _{n \rightarrow \infty}\left\|v_{n}-v\right\|_{L^{\infty}}\|f\|_{M(\Omega)}=0 .
$$

Além disso, como:

$$
|\nabla u|^{p-2} \nabla u \in L^{r}(\Omega),
$$

temos que:

$$
\lim _{n \rightarrow \infty} \int_{\Omega}|\nabla u|^{p-2} \nabla u \cdot \nabla v_{n}=\int_{\Omega}|\nabla u|^{p-2} \nabla u \cdot \nabla v .
$$

Combinando-se (3.34) - (3.37), obtemos que:

$$
\left\{\begin{array}{l}
\int_{\Omega}|\nabla u|^{p-2} \nabla u \cdot \nabla v=\int_{\Omega} f v \quad \forall v \in W_{0}^{1, r^{\prime}}(\Omega) \text { e } \forall r^{\prime}>N \\
|\nabla u|^{p-2} \nabla u \in L^{r}(\Omega) \quad \forall r \in\left[1, \frac{N}{N-1}\right) .
\end{array}\right.
$$

Sendo assim, no Teorema 3.1.4, provamos a existência de uma solução mais regular do que dado na Definição 3.1.1. 


\subsection{Continuidade para Regularidades Intermediárias}

Na seção anterior, obtivemos um resultado de existência e regularidade de soluções para o problema

$$
(P) \quad\left\{\begin{array}{rlll}
-\Delta_{p} u & = & f & \text { em }
\end{array}\right.
$$

para $f \in M(\Omega)$, o que inclui naturalmente o caso $f \in L^{1}(\Omega)$.

Paralelamente, no Teorema 2.2.5, pág. 36, obtivemos um resultado análogo para o caso em que $f \in W^{-1, p^{\prime}}$.

A questão que surge é o que aconteceria com as soluções de $(P)$ para $f$ em um espaço intermediário.

Na realidade, estamos interessados em entender o comportamento das soluções deste problema quando $f \in W^{-1, p^{\prime}} \cap L^{m}$, para $m>1$.

Veja que assim, usando uma estratégia análoga ao do Teorema 3.1.4, recuperaríamos o caso em que $f \in L^{m}$.

Assim, sendo o caso de interesse se restringe a quando:

$$
1<m<\left(p^{*}\right)^{\prime}
$$

De fato, se $m \geq\left(p^{*}\right)^{\prime}$, então por Imersão de Sobolev,

$$
L^{m}(\Omega) \hookrightarrow L^{\left(p^{*}\right)^{\prime}}(\Omega) \hookrightarrow W^{-1, p^{\prime}}(\Omega)
$$

de modo que

$$
W^{-1, p^{\prime}}(\Omega) \cap L^{m}(\Omega)=W^{-1, p^{\prime}}(\Omega)
$$

e assim, bastaria usar o Teorema 2.1.6 (cf. Leray-Lions [13]).

Portanto, nesta seção, investigaremos o problema $(P)$ para $f \in W^{-1, p^{\prime}} \cap L^{m}$, onde $1<m<$ $\left(p^{*}\right)^{\prime}$.

Vale ainda notarmos que:

$$
\left(p^{*}\right)^{\prime}=\frac{N P}{N p-N+p} .
$$

Logo, nossa análise fará sentido quando $p$ satisfazer a seguinte restrição adicional:

$$
2-\frac{1}{N}<p<N
$$


De fato, se $p=n$, então $\left(p^{*}\right)^{\prime}=1$. A próxima figura resume de maneira simples o argumento acima

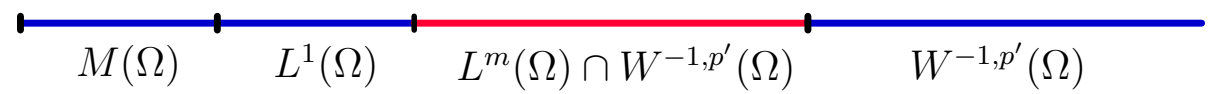

Por simplicidade de notação, tomaremos

$$
\bar{m}=\left(p^{*}\right)^{\prime}
$$

Neste contexto, vale o seguinte resultado de regularidade para os casos intermediários.

Lema 3.2.1. Sejam $p \in\left(2-\frac{1}{N}, N\right)$ e $1<m<\bar{m}$. Então, se $f \in L^{m}(\Omega) \cap W^{-1, p^{\prime}}(\Omega)$ para cada $B>0$ tal que $\|f\|_{L^{m}} \leq B$, existe $C=C(p, q, \Omega, B)>0$ tal que

$$
\|u\|_{W_{0}^{1, q}} \leq C
$$

onde $1 \leq q<m^{*}(p-1)$, para toda u solução fraca de $(P)$.

Demonstração. A prova deste lema é análoga a do Lema 3.1.2. Para conveniência do leitor, apresentamos aqui os detalhes.

Sendo assim, consideramos $n \in \mathbb{N} \cup\{0\}$ e os seguintes conjuntos:

$$
\begin{aligned}
& B_{n}=\{x \in \Omega ; n \leq|u(x)| \leq n+1\} ; \\
& E_{n}=\{x \in \Omega ; n<|u(x)|\} .
\end{aligned}
$$

Além disso, tomaremos mais uma vez $\psi: \mathbb{R} \longrightarrow \mathbb{R}$ dada por:

$$
\psi(s)=\left\{\begin{array}{cll}
1 & \text { se } & s>n+1 \\
s-n & \text { se } & n \leq s \leq n+1 \\
0 & \text { se } & -n \leq s \leq n \\
s+n & \text { se } & -n-1 \leq s \leq-n \\
-1 & \text { se } & s<-n-1 .
\end{array}\right.
$$

Consideramos $f \in L^{m}(\Omega) \cap W^{-1, p^{\prime}}(\Omega)$, e então, pelo Teorema 2.2.5, pág. 38, existe $u \in$ $W_{0}^{1, p}(\Omega)$ tal que:

$$
\int_{\Omega}|\nabla u|^{p-2} \nabla u \cdot \nabla \varphi=\int_{\Omega} f \varphi, \quad \forall \varphi \in W_{0}^{1, p}(\Omega)
$$


Tomando em (3.39) $\varphi=\psi(u)$, o que é possível pois $\psi(u) \in W_{0}^{1, p}(\Omega)$, obtemos que:

$$
\int_{B_{n}}|\nabla u|^{p} \leq \int_{E_{n}} f \psi(u)
$$

De fato, sejam:

$$
D_{n}=\{x \in \Omega ;|u(x)|=n\} \text { e } A_{n}=\{x \in \Omega ;|u(x)|>n+1\} .
$$

Claramente, temos $A_{n} \cup B_{n}=D_{n} \cup E_{n}$, além do mais,

$$
A_{n} \cap B_{n}=\phi .
$$

Assim,

$$
\begin{aligned}
\int_{B_{n} \cup A_{n}} \psi^{\prime}(u)|\nabla u|^{p} & =\int_{D_{n} \cup E_{n}} \psi^{\prime}(u)|\nabla u|^{p} \\
& =\int_{D_{n} \cup E_{n}} f \psi(u) \\
& =\int_{B_{n} \cup A_{n}} f \psi(u) .
\end{aligned}
$$

Entretanto, como $\operatorname{med}\left(A_{n} \cap B_{n}\right)=0$, segue que

$$
\begin{aligned}
\int_{B_{n} \cup A_{n}} \psi^{\prime}(u)|\nabla u|^{p} & =\int_{B_{n}} \psi^{\prime}(u)|\nabla u|^{p}+\int_{A_{n}} \psi^{\prime}(u)|\nabla u|^{p} \\
& =\int_{B_{n}} \psi^{\prime}(u)|\nabla u|^{p},
\end{aligned}
$$

pois $\psi^{\prime}(u)=0$ em $A_{n}$.

De modo análogo, como $\operatorname{med}\left(D_{n} \cap E_{n}\right)=0$ e $\psi^{\prime}(u)=0$ em $D_{n}$, temos:

$$
\int_{D_{n} \cup E_{n}} \psi^{\prime}(u)|\nabla u|^{p}=\int_{E_{n}} \psi^{\prime}(u)|\nabla u|^{p} .
$$

Combinando (3.41) e (3.42), obtemos que:

$$
\begin{aligned}
\int_{B_{n}}|\nabla u|^{p} & =\int_{B_{n}} \psi^{\prime}(u)|\nabla u|^{p} \\
& \leq \int_{E_{n}} f \psi(u),
\end{aligned}
$$

afinal $\psi^{\prime}(u)=1$ em $B_{n}$, provando então (3.40).

Prosseguindo, pela Desigualdade de Hölder, obtemos que:

$$
\int_{E_{n}} f \psi(u) \leq\|f\|_{L^{m}\left(E_{n}\right)}\left(\operatorname{med}\left(E_{n}\right)\right)^{\frac{1}{m^{\prime}}} \leq B\left(\operatorname{med}\left(E_{n}\right)\right)^{\frac{1}{m^{\prime}}} .
$$


Logo, por (3.40) e (3.43), obtemos que:

$$
\int_{B_{n}}|\nabla u|^{p} \leq B\left(\operatorname{med}\left(E_{n}\right)\right)^{\frac{1}{m^{\prime}}}
$$

Agora, observe que:

$$
q<p
$$

De fato, $q<m^{*}(p-1)$ e $m \leq \bar{m}$. Logo, $m^{*} \leq(\bar{m})^{*}$.

Porém,

$$
(\bar{m})^{*}=\left(\left(p^{*}\right)^{\prime}\right)^{*}=\frac{N\left(p^{*}\right)^{\prime}}{N-\left(p^{*}\right)^{\prime}}=\frac{N p}{N p-N}=\frac{p}{p-1} .
$$

Portanto, $q<\frac{p}{p-1}(p-1)$, o que mostra a validade de (3.45). Assim, como $q \in[1, p)$, pela desigualdade de Hölder, segue que:

$$
\int_{B_{n}}|\nabla u|^{q} \leq\left(\int_{B_{n}}|\nabla u|^{p}\right)^{q / p}\left(\operatorname{med}\left(B_{n}\right)\right)^{(p-q) / p} .
$$

Agora, como $q<p$ e $u \in W_{0}^{1, p}(\Omega)$, temos pelo Teorema 1.2.8, pág. 22, que $W_{0}^{1, q}(\Omega) \hookrightarrow$ $L^{q^{*}}(\Omega)$, e assim, segue que $u \in L^{q^{*}}(\Omega)$ por (3.47). Além disso, pela escolha de $B_{n}$, temos:

$$
\operatorname{med}\left(B_{n}\right) \leq \frac{1}{n^{q^{*}}} \int_{B_{n}}|u|^{q^{*}}
$$

Usando o mesmo argumento acima para o conjunto $E_{n}$, segue que:

$$
\operatorname{med}\left(E_{n}\right) \leq \frac{1}{n^{q^{*}}} \int_{E_{n}}|u|^{q^{*}} \leq \frac{1}{n^{q^{*}}} \int_{\Omega}|u|^{q^{*}}
$$

Combinando-se (3.44), (3.47), (3.48) e (3.49), obtemos que:

$$
\begin{aligned}
\int_{B_{n}}|\nabla u|^{q} & \leq B^{q / p}\left(\operatorname{med}\left(E_{n}\right)\right)^{q / p m^{\prime}}\left(\operatorname{med}\left(B_{n}\right)\right)^{(p-q) / p} \\
& \leq c_{1}\left(\frac{1}{n^{q^{*}}} \int_{\Omega}|u|^{q^{*}}\right)^{q / p m^{\prime}}\left(\frac{1}{n^{q^{*}}} \int_{B_{n}}|u|^{q^{*}}\right)^{(p-q) / p} \\
& =c_{1} \frac{1}{n^{q^{*}\left(q / p m^{\prime}\right)}}\|u\|_{L^{q^{*}(\Omega)}}^{q^{*}\left(q / p m^{\prime}\right)} \frac{1}{n^{q^{*}(p-q / p)}}\left(\int_{B_{n}}|u|^{q^{*}}\right)^{(p-q) / p} \\
& =c_{1}\|u\|_{L^{q^{*}(\Omega)}}^{q^{*}\left(q / p m^{\prime}\right)}\left(\int_{B_{n}}|u|^{q^{*}}\right)^{(p-q) / p} \frac{1}{n^{\left(q^{*} / p\right)\left(q / m^{\prime}+p-q\right)}} .
\end{aligned}
$$

Portanto,

$$
\int_{B_{n}}|\nabla u|^{q} \leq c_{1}|| u \|_{L^{q^{*}}(\Omega)}^{q^{*}\left(q / p m^{\prime}\right)}\left(\int_{B_{n}}|u|^{q^{*}}\right)^{(p-q) / p} \frac{1}{n^{\left(q^{*} / p\right)\left(q / m^{\prime}+p-q\right)}},
$$


onde $c_{1}=B^{q / p}$.

Tomando-se o somatório em (3.50), obtemos que:

$$
\sum_{n=1}^{\infty} \int_{B_{n}}|\nabla u|^{q} \leq c_{1}\|u\|_{L^{q^{*}}(\Omega)}^{q^{*}\left(q / p m^{\prime}\right)} \sum_{n=1}^{\infty}\left(\int_{B_{n}}|u|^{q^{*}}\right)^{(p-q) / p} \frac{1}{n^{\left(q^{*} / p\right)\left(q / m^{\prime}+p-q\right)}},
$$

Aplicando-se Hölder para séries, segue que:

$$
\sum_{n=1}^{\infty} \int_{B_{n}}|\nabla u|^{q} \leq c_{1}\|u\|_{L^{q^{*}}}^{q^{*}\left(q / p m^{\prime}\right)}\left(\sum_{n=1}^{\infty} \int_{B_{n}}|u|^{q^{*}}\right)^{(p-q) / p}\left(\sum_{n=1}^{\infty} \frac{1}{n^{\left(q^{*} / p\right)\left(q / m^{\prime}+p-q\right)}}\right)^{q / p} .
$$

Porém, para $G_{1}=\{x \in \Omega:|u(x)| \leq 1\}$, escolhendo-se $\psi$ como a mesma de (3.2), temos que:

$$
\int_{G_{1}}|\nabla u|^{p} \leq\|f\|_{L^{1}} \leq B
$$

Assim,

$$
\int_{G_{1}}|\nabla u|^{q} \leq B^{p / q}
$$

Além disso, de maneira análoga (3.47), temos:

$$
\int_{G_{1}}|\nabla u|^{q} \leq\left(\int_{G_{1}}|\nabla u|^{p}\right)^{q / p}\left(\operatorname{med}\left(G_{1}\right)\right)^{(p-q) / p} \leq c_{1}(\operatorname{med}(\Omega))^{(p-q) / p}=c_{2} .
$$

Como $\Omega \subset \cup_{n=1}^{\infty} B_{n} \cup G_{1}$, segue da subaditividade da medida de Lebesgue que:

$$
\begin{aligned}
\int_{\Omega}|\nabla u|^{q} & \leq \int_{G_{1}}|\nabla u|^{q}+\sum_{n=1}^{\infty} \int_{B_{n}}|\nabla u|^{q} \\
& \leq c_{2}+c_{1}|| u \|_{L^{q^{*}}}^{q^{*}\left(q / p m^{\prime}\right)}\left(\sum_{n=1}^{\infty} \int_{B_{n}}|u|^{q^{*}}\right)^{(p-q) / p}\left(\sum_{n=1}^{\infty} \frac{1}{n^{\left(q^{*} / p\right)\left(q / m^{\prime}+p-q\right)}}\right)^{q / p} .
\end{aligned}
$$

Observando que:

$$
\sum_{n=1}^{\infty} \int_{B_{n}}|u|^{q^{*}} \leq \int_{\Omega}|u|^{q^{*}}
$$

temos:

$$
\int_{\Omega}|\nabla u|^{q} \leq c_{2}+c_{1}\|u\|_{L^{q^{*}}}^{q^{*}\left(q / p m^{\prime}+(p-q) / p\right)}\left(\sum_{n=1}^{\infty} \frac{1}{n^{\left(q^{*} / p\right)\left(q / m^{\prime}+p-q\right)}}\right)^{q / p} .
$$

Pelo Teorema 1.2.8, pág. 22, e tomando $c_{3}=\max \left\{c_{1}, c_{2}\right\}$, segue que:

$$
\|u\|_{L^{q^{*}}}^{q} \leq c_{3} K\left(1+\|u\|_{L^{q^{*}}}^{q *\left(p / p m^{\prime}+(p-q) / p\right)}\left(\sum_{n=1}^{\infty} \frac{1}{n^{\left(q^{*} / q\right)(p-q / m)}}\right)^{q / p}\right),
$$

onde $K$ é a constante de Sobolev. 
Agora, observe que:

$$
q^{*}\left(\frac{q}{p m^{\prime}}+\frac{p-q}{p}\right)<q
$$

e

$$
\frac{q^{*}}{q}\left(p-\frac{q}{m}\right)>1
$$

De fato, para provar (3.57) observe que por hipótese $1<p<N$ e $m<\bar{m}=N p /(N p-N+p)$. Assim, se $p<N$, temos $p^{2}<N p-N+p$. Com isso, segue que:

$$
\frac{N p^{2}}{N p-N+p}<N
$$

Mas assim, obtemos $p m<N$. Multiplicando por $-q^{2}$ e somando $q N$ por ambos lados, segue que:

$$
q N-\frac{q^{2} N}{p m}<q(N-q)
$$

Note que:

$$
q N-\frac{q^{2} N}{p m}<q(N-q)=q N\left(\frac{p m-q}{p m}\right)<q(N-q)
$$

$\log \mathrm{O}$

$$
\frac{q N}{N-q}\left(\frac{q}{p}\left(1-\frac{1}{m}\right)+\frac{p-1}{p}\right)<q
$$

Portanto, segue (3.57).

Agora, para provar (3.58), como por hipótese $q<(p-1) m^{*}=(p-1)(m N / N-m)$, temos $N m p>N m-q m+q N . \mathrm{E}$ assim,

$$
\frac{N m p}{N m-q m}>\frac{N m-q m+q N}{N m-q m} .
$$

Com isso, observe que

$$
\frac{N m p}{N m-q m}-\frac{q N}{N m-q m}>1 .
$$

Multiplicando por $q$ ambos os lados, segue que:

$$
\frac{q N m p}{N m-q m}-\frac{q^{2} N}{N m-q m}>q \text {. }
$$

Assim, segue (3.58).

Neste caso, obtemos por (3.56) que:

$$
\|u\|_{L^{q^{*}}}^{q} \leq c_{4}\left(1+\|u\|_{L^{q^{*}}}^{\sigma q}\right)
$$


onde

$$
c_{4}=\max \left\{c_{3} K, c_{3} K\left(\sum_{n=1}^{\infty} \frac{1}{\left.n^{\left(q^{*} / q\right.}\right)(p-q / m)}\right)^{q / p}\right\}
$$

$\mathrm{e}$

$$
\sigma=\frac{q *\left(q / p m^{\prime}+(p-q) / p\right)}{q} \in(0,1) .
$$

Assim, usando a desigualdade de Young:

$$
\|u\|_{L^{q^{*}}}^{q} \leq c_{4}\left(1+\|u\|_{L^{q^{*}}}^{q} \varepsilon+c(\varepsilon)\right) .
$$

Tomando $\varepsilon=\frac{1}{2 c_{4}}$, temos que:

$$
\|u\|_{L^{q^{*}}}^{q} \leq c_{5}
$$

onde $c_{5}=2 c_{4}\left(1+c\left(\frac{1}{2 c_{4}}\right)\right)$.

Assim, o resultado segue.

Teorema 3.2.2. Para $p \in(2-1 / N, N)$, considere $f \in L^{m}(\Omega)$, onde $m \in(1, \bar{m})$. Então o problema $(P)$ possui uma solução tal que, para cada $q \in\left[1,(p-1) m^{*}\right), u \in W_{0}^{1, q}(\Omega)$.

Demonstração. Basta usar o Lema 3.2.1 e repetir o argumento do Teorema 3.1.4.

Observe que o resultado do teorema acima, nos fornece a regularidade para a solução com dependência contínua na ordem de integrabilidade do termo forçante $f$. De fato, o Teorema 2.1.6 nos garante que $u \in W_{0}^{1, p}(\Omega)$ caso $f \in L^{(p)^{*}}(\Omega)=L^{\bar{m}}(\Omega)$.

De maneira complementar, pelo Teorema 3.1.4, tais que se $f \in L^{1}(\Omega)$ então:

$$
u \in W_{0}^{1, q}(\Omega) \quad \text { para } \quad q<\frac{N}{N-1}(p-1) .
$$

Porém, com o teorema acima em mãos, concluímos que se $f \in L^{m}(\Omega)$, então:

$$
u \in W_{0}^{1, q}(\Omega) \quad \text { onde } \quad 1 \leq q<m^{*}(p-1) .
$$

Veja que se $m=1$, então:

$$
m^{*}(p-1)=\frac{N}{N-1}(p-1) .
$$

Além disso, se $m=\left(p^{*}\right)^{\prime}$ então:

$$
m^{*}(p-1)=p
$$

Neste sentido, temos continuidade da regularidade nos dois casos extremos previstos no Teorema 3.2.2. 


\subsection{Efeitos das Perturbações Semilineares}

Nesta seção estamos interessados em estudar a existência de soluções fracas para o seguinte problema:

$$
\left(P^{\prime}\right) \quad\left\{\begin{aligned}
-\Delta_{p} u+g(\cdot, u) & =f, & \text { em } \Omega, \\
u & =0, & \text { em } \partial \Omega
\end{aligned}\right.
$$

onde $f \in M(\Omega)$ ou $f \in L^{1}(\Omega)$ e a função $g$ satisfaz as seguintes hipóteses:

$g(x, s)$ é mensurável em $x \in \Omega, \forall s \in \mathbb{R}$ e contínua em $s \in \mathbb{R}$, q.t.p em $x \in \Omega$;

$$
g(x, s) s \geq 0 \forall s \in \mathbb{R} \text {, q.t.p em } x \in \Omega ;
$$

existem $b_{1}, b_{2}, \delta$ com $b_{1} \in L_{l o c}^{1}(\Omega), b_{2} \in L_{l o c}^{\infty}(\Omega)$,

$$
\delta<N(p-1) /(N-p) \text {, tal que }|g(x, s)| \leq b_{1}(x)+b_{2}(x)|s|^{\delta}
$$

q.t.p. em $x$, para todo número real $s$.

Observamos que com a hipótese (3.62), a função $g(x, s)$ está em $L_{l o c}^{1}(\Omega)$, para cada $s \in \mathbb{R}$. A seguir, definimos o conceito de solução fraca para o problema $\left(P^{\prime}\right)$.

Definição 3.3.1. Dizemos que uma função u é solução fraca de $\left(P^{\prime}\right)$ se satisfaz:

$$
\left\{\begin{array}{l}
u \in W_{0}^{1,1}(\Omega),|\nabla u|^{p-2} \nabla u \in L_{l o c}^{1}(\Omega), g(\cdot, u) \in L_{l o c}^{1}(\Omega) e \\
\int_{\Omega}|\nabla u|^{p-2} \nabla u \cdot \nabla \varphi+\int_{\Omega} g(x, u) \varphi=\int_{\Omega} f \varphi, \quad \text { para toda } \varphi \in C_{0}^{\infty}(\Omega) .
\end{array}\right.
$$

O método utilizado nesta seção para encontrarmos existência de soluções fracas para o problema $\left(P^{\prime}\right)$ é muito semelhante ao usado na Seção 3.1.

Com as hipóteses (3.60)-(3.62) sobre a $g$ do problema $\left(P^{\prime}\right)$, temos o seguinte resultado:

Teorema 3.3.2. Seja g satisfazendo (3.60), (3.61) e (3.62), e $f \in M(\Omega)$. Então existe uma solução fraca u de $\left(P^{\prime}\right)$ (ou seja, uma u que satisfaz (3.63)).

Demonstração. Seja $f \in M(\Omega)$ com $\|f\|_{M(\Omega)}=B$. Novamente, combinando o Corolário 1.1.30, pág. 17, e pelo Teorema 1.1.35, pág. 19, existe $\left(f_{n}\right) \subset L^{p^{\prime}}(\Omega) \subset L^{1}(\Omega) \cap W^{-1, p^{\prime}}(\Omega)$ tal que

$$
f_{n} \longrightarrow f \quad \text { no sentido das distribuições. }
$$


Nossa estratégia para provar o Teorema 3.3.2 será dividida em 3 passos. O primeiro passo é dedicado a obtenção de soluções fracas para um problema aproximado. Já o segundo passo, obteremos estimativas sobre a solução do problema aproximado para por fim, no terceiro passo usarmos o processo do limite.

\section{Passo 1: Problema Aproximado}

Seja $g$ satisfazendo (3.60) e (3.61). Para cada $n \in \mathbb{N}$, definimos:

$$
g_{n}(x, s)= \begin{cases}g(x, s) & \text { se }|g(x, s)| \leq n, x \in \Omega, s \in \mathbb{R} \\ n & \text { se } g(x, s)>n, x \in \Omega, s \in \mathbb{R} \\ -n & \text { se } g(x, s)<-n, x \in \Omega, s \in \mathbb{R} .\end{cases}
$$

Tomando-se $f_{n} \in W^{-1, p^{\prime}}(\Omega)$, para cada $n \in \mathbb{N}$, consideramos o seguinte problema:

$$
\left\{\begin{aligned}
-\Delta_{p} u+g_{n}(\cdot, u) & =f_{n}, & \text { em } \Omega \\
u & =0, & \text { em } \partial \Omega
\end{aligned}\right.
$$

Note que para cada $n \in \mathbb{N}, g_{n}$ satisfaz as hipóteses (3.60) e (3.61). De fato, separamos nos três casos:

- $|g(x, s)| \leq n$

Neste caso, temos que $g_{n}(x, s)=g(x, s)$, e assim, o resultado segue de imediato.

- $g(x, s)>n$

Temos que $g_{n}(x, s)=n$. Note que a hipótese (3.60) é satisfeita também de imediato. Para ver (3.61), observe que como $g(x, s)>n$, então necessariamente $s \geq 0$ pela hipótese sobre $g$, e assim, $g_{n}(x, s) s=n s \geq 0$.

- $g(x, s)<-n$

Como neste caso $g_{n}(x, s)=-n$, as hipóteses (3.60) e (3.61) são satisfeitas. Para ver (3.61), observe que como $g(x, s)<-n$, então necessariamente $s \leq 0, \log 0 g_{n}(x, s) s=-n s \geq 0$.

Com isso, ainda notamos que

$$
\left|g_{n}(x, s)\right| \leq n, \text { para cada } n \in \mathbb{N},
$$


e então pelo Teorema 2.2.6, pág. 40, temos que para cada $n \in \mathbb{N}$, existe solução fraca para (3.65), ou seja, existe $u_{n} \in W_{0}^{1, p}(\Omega)$ tal que:

$$
\int_{\Omega}\left|\nabla u_{n}\right|^{p-2} \nabla u_{n} \cdot \nabla \varphi+\int_{\Omega} g_{n}\left(x, u_{n}\right) \varphi=\int_{\Omega} f_{n} \varphi, \text { para toda } \varphi \in W_{0}^{1, p}(\Omega) .
$$

\section{Passo 2: Estimativas do Problema Aproximado}

Nosso objetivo é obter estimativas para a solução $\left(u_{n}\right)$. Para tanto, precisamos estabelecer as afirmações seguintes:

\section{Afirmação 3.3.1.}

$$
\begin{aligned}
& \int_{\left\{\left|u_{n}\right|>t\right\}}\left|g_{n}\left(x, u_{n}\right)\right| \leq \int_{\left\{\left|u_{n}\right|>t\right\}}\left|f_{n}\right|, \text { para todo inteiro } n e \\
& \quad \text { todo } t \in \mathbb{R}^{+}, \text {onde }\left\{\left|u_{n}\right|>t\right\}=\left\{x \in \Omega:\left|u_{n}(x)\right|>t\right\} .
\end{aligned}
$$

Prova da Afirmação 3.3.1:

Seja $\psi_{i}$ uma sequência de funções suaves a valores reais. Note que $\psi_{i}\left(u_{n}\right) \in W_{0}^{1, p}(\Omega)$ (veja [14], Teorema 7.8, pág. 153), e assim, tomando-se $\psi_{i}\left(u_{n}\right)$ como função teste em (3.66), temos

$$
\int_{\Omega} g_{n}\left(x, u_{n}\right) \psi_{i}\left(u_{n}\right) \leq \int_{\Omega} f_{n} \psi_{i}\left(u_{n}\right) .
$$

Agora, tomamos a sequência $\psi_{i}$ da seguinte forma:

$$
\psi_{i}(s)= \begin{cases}1 & \text { se } s>t \\ \left(\frac{s}{t}\right)^{i} & \text { se } 0 \leq s \leq t \\ -\left(-\frac{s}{t}\right)^{i} & \text { se }-t \leq s \leq 0 \\ -1 & \text { se } s<-t\end{cases}
$$

Note que:

$$
\lim _{i \rightarrow \infty} \psi_{i}(s)= \begin{cases}1 & \text { se } s>t \\ 0 & \text { se }-t \leq s \leq t \\ -1 & \text { se } s<-t\end{cases}
$$

Com isso, temos: 


$$
\lim _{i \rightarrow \infty} \psi_{i}\left(u_{n}(x)\right)= \begin{cases}1 & \text { se } u_{n}(x)>t \\ 0 & \text { se }-t \leq u_{n}(x) \leq t \\ -1 & \text { se } u_{n}(x)<-t .\end{cases}
$$

Então:

$$
\begin{aligned}
\lim _{i \rightarrow \infty} g_{n}\left(x, u_{n}\right) \psi_{i}\left(u_{n}(x)\right) & = \begin{cases}g_{n}\left(x, u_{n}\right) & \text { se } u_{n}(x)>t \\
0 & \text { se }-t \leq u_{n}(x) \leq t \\
-g_{n}\left(x, u_{n}\right) & \text { se } u_{n}(x)<-t .\end{cases} \\
& = \begin{cases}\left|g_{n}\left(x, u_{n}\right)\right| & \text { se }\left|u_{n}(x)\right|>t \\
0 & \text { se }\left|u_{n}(x)\right| \leq t .\end{cases}
\end{aligned}
$$

Pelo Lema de Fatou, temos:

$$
\begin{aligned}
\int_{\Omega} \liminf _{i \rightarrow \infty} g_{n}\left(x, u_{n}\right) \psi_{i}\left(u_{n}\right) & \leq \liminf _{i \rightarrow \infty} \int_{\Omega} g_{n}\left(x, u_{n}\right) \psi_{i}\left(u_{n}\right) \\
& \leq \liminf _{i \rightarrow \infty} \int_{\Omega} f_{n} \psi_{i}\left(u_{n}\right) \\
& \leq \int_{\Omega}\left|f_{n}\right| .
\end{aligned}
$$

Logo:

$$
\int_{\left\{\left|u_{n}\right|>t\right\}}\left|g_{n}\left(x, u_{n}\right)\right| \leq \int_{\left\{\left|u_{n}\right|>t\right\}}\left|f_{n}\right|, \quad \text { para todo } t \in \mathbb{R}^{+} .
$$

Portanto, a Afirmação 3.3.1 está estabelecida.

A próxima afirmação, nos dará um resultado de compacidade para a sequência $\left(u_{n}\right)$.

\section{Afirmação 3.3.2.}

A sequência $\left(u_{n}\right)$ é relativamente compacta em $W_{0}^{1, q}(\Omega)$ para todo $q$ em $[1,(N /(N-1))(p-1))$, onde $p \in(2-1 / N, N]$.

Prova da Afirmação 3.3.2:

Tomando-se $t \rightarrow 0^{+}$e aplicando-se novamente o Lema de Fatou, segue que:

$$
\left\|g_{n}\left(\cdot, u_{n}\right)\right\|_{L^{1}} \leq\left\|f_{n}\right\|_{L^{1}}
$$

Tomando-se $h_{n}=f_{n}-g_{n}\left(\cdot, u_{n}\right)$, obtemos que: 


$$
\begin{aligned}
\int_{\Omega} f_{n}-g_{n}\left(\cdot, u_{n}\right) & \leq \int_{\Omega}\left|f_{n}-g_{n}\left(\cdot, u_{n}\right)\right| \\
& \leq \int_{\Omega}\left|f_{n}\right|+\left|g_{n}\left(\cdot, u_{n}\right)\right| \\
& \leq \int_{\Omega}\left|f_{n}\right|+\int_{\Omega}\left|f_{n}\right| \\
& \leq 2 B .
\end{aligned}
$$

Portanto

$$
\left\|h_{n}\right\|_{L^{1}} \leq 2 B, \quad h_{n} \in L^{1}(\Omega) \cap W^{-1, p^{\prime}}(\Omega)
$$

Note que $u_{n}$ é a solução de (3.1) tomando-se $f=h_{n}$.

Agora, por (3.69) e repetindo o mesmo argumento que no Lema 3.1.2, segue que $\left(u_{n}\right)$ é limitada em $W_{0}^{1, q}(\Omega)$, para todo $1 \leq q<(N /(N-1))(p-1)$. Com isso, podemos também repetir o mesmo argumento da demonstração do Teorema 3.1.4 e, assim, obter a Afirmação 3.3.2.

\section{Passo 3: Processo Limite}

Estabelecidos os passos 1 e 2, podemos concluir a prova do Teorema 3.3.2.

Como vimos, temos que para cada $n \in \mathbb{N}$, existe $u_{n} \in W_{0}^{1, p}(\Omega)$ satisfazendo:

$$
\int_{\Omega}\left|\nabla u_{n}\right|^{p-2} \nabla u_{n} \cdot \nabla \varphi+\int_{\Omega} g\left(x, u_{n}\right) \varphi=\int_{\Omega} f_{n} \varphi, \text { para toda } \varphi \in C_{0}^{\infty}(\Omega) .
$$

Por (3.3.2), segue que a sequência $\left(u_{n}\right)$ é relativamente compacta em $W_{0}^{1, q}(\Omega)$ para $1 \leq q<$ $(N /(N-1))(p-1)$. Então, existe $u \in W_{0}^{1, q}(\Omega)$, tal que menos de subsequências, temos que:

$$
\begin{array}{rlrl}
u_{n} & \rightarrow u & & \text { em } W_{0}^{1, q}(\Omega), 1 \leq q<\frac{N}{N-1}(p-1) \\
u_{n} \rightarrow u & & \text { q.t.p. em } \Omega \\
\left|\nabla u_{n}\right|^{p-2} \nabla u_{n} & \rightarrow|\nabla u|^{p-2} \nabla u & & \text { em } L^{r}(\Omega), 1 \leq r<\frac{N}{N-1} .
\end{array}
$$

Observando que a última hipótese de (3.71) é obtida pelo mesmo argumento que usamos para obter (3.27).

Além disso, como $W_{0}^{1, q}(\Omega) \hookrightarrow L^{q^{*}}(\Omega)$, para $1 \leq q<N$, segue que:

$$
u_{n} \rightarrow u \quad \text { em } L^{r}(\Omega), 1 \leq r<\frac{N(p-1)}{N-p} .
$$


Agora, note que $q^{*}<N(p-1) / N-p$. De fato, por hipótese $q<N(p-1) / N-1$, e assim $q N-q<p N-N$. Subtraindo $p q$ e somando $q$ em ambos os lados da desigualdade, segue que:

$$
q N-p q<p N-p q-N+q
$$

Rearranjando a desigualdade acima, obtemos:

$$
q<\frac{(p-1)(N-q)}{N-p}
$$

Multiplicando por $N$ ambos os lado da desigualdade acima, temos:

$$
\frac{N q}{N-q}<\frac{N(p-1)}{N-p}
$$

Logo:

$$
q^{*}<\frac{N(p-1)}{N-p} .
$$

Agora, por (3.71) como $u_{n}(x) \rightarrow u(x)$ q.t.p em $\Omega$, e além disso, $g$ sendo contínua no seu segundo argumento, segue que:

$$
g_{n}\left(\cdot, u_{n}\right) \rightarrow g(\cdot, u) \quad \text { q.t.p. em } \Omega
$$

Ainda, note que:

$$
\left|g_{n}\left(\cdot, u_{n}\right)-g(\cdot, u)\right| \leq\left|g_{n}\left(\cdot, u_{n}\right)\right|+|g(\cdot, u)| \leq 2 b_{1}(x)+b_{2}(x)\left(\left|u_{n}\right|^{\delta}+|u|^{\delta}\right)
$$

e como

$$
\left|u_{n}\right|^{\delta} \leq\left(\left|u_{n}-u\right|+|u|\right)^{\delta} \leq 2^{\delta-1}\left(\left|u_{n}-u\right|^{\delta}+|u|^{\delta}\right),
$$

temos

$$
\left|g_{n}\left(\cdot, u_{n}\right)-g(\cdot, u)\right| \leq 2 b_{1}(x)+b_{2}(x)\left(2^{\delta-1}\left|u_{n}-u\right|^{\delta}+2^{\delta-1}|u|^{\delta}+|u|^{\delta}\right) .
$$

Assim, pela hipótese (3.62) com $\left|u_{n}-u\right|^{\delta}$ e $|u|^{\delta}$ sendo limitados, temos pelo Teorema da Convergência Dominada de Lebesgue que:

$$
g_{n}\left(\cdot, u_{n}\right) \rightarrow g(\cdot, u) \quad \text { em } L_{l o c}^{1}(\Omega)
$$

Pelos resultados em (3.71) e (3.73) junto com o fato de $\left(f_{n}\right)$ convergir para $f$ no sentido das distribuições, podemos então passar o limite em (3.70) e obter (3.63). Para concluirmos o 
Teorema 3.3.2, ainda demos mostrar que $g(\cdot, u) \in L^{1}(\Omega)$. Porém, pelo Lema de Fatou e pela estimativa obtida em (3.68), temos:

$$
\begin{aligned}
\left.\int_{\Omega} \mid g_{(\cdot,} u\right)\left|=\int_{\Omega} \liminf _{n \rightarrow \infty}\right| g_{n}\left(\cdot, u_{n}\right) \mid & \leq \liminf _{n \rightarrow \infty} \int_{\Omega}\left|g\left(\cdot, u_{n}\right)\right| \\
& \leq \liminf _{n \rightarrow \infty} \int_{\Omega}\left|f_{n}\right| \\
& \leq\|f\|_{M(\Omega)} .
\end{aligned}
$$

Portanto, $\|g(\cdot, u)\|_{L^{1}} \leq B=\|f\|_{M(\Omega)}$, e assim, a demonstração do Teorema 3.3.2 está completa, isto é, temos:

$$
\begin{array}{lr}
u \in W_{0}^{1, q}(\Omega), & \text { para todo } 1 \leq q<\frac{N}{N-1}(p-1), \\
|\nabla u|^{p-2} \nabla u \in L^{r}(\Omega) & \text { para todo } 1 \leq r<\frac{N}{N-1} \\
g(\cdot, u) \in L^{1}(\Omega) & \\
\int_{\Omega}|\nabla u|^{p-2} \nabla u \cdot \nabla \varphi+\int_{\Omega} g(x, u) \varphi=\int_{\Omega} f \varphi, \\
& \text { para toda } \varphi \in \bigcup_{r^{\prime}>N} W_{0}^{1, r^{\prime}}(\Omega) .
\end{array}
$$

Se colocarmos uma hipótese mais forte sobre a função $f$ podemos enfraquecer a hipótese (3.62). De fato, se $f \in L^{1}(\Omega)$, então trocando a hipótese (3.62) pela hipótese:

$$
\sup \{|g(x, s)|,|s| \leq t\} \in L_{l o c}^{1}(\Omega), \forall t \in \mathbb{R}^{+}
$$

temos o seguinte resultado:

Teorema 3.3.3. Seja $f \in L^{1}(\Omega)$ e g satisfazendo (3.60), (3.61) e (3.75). Então existe uma solução fraca u para o problema $\left(P^{\prime}\right)$.

Demonstração. Seja $f \in L^{1}(\Omega)$, e além disso, $g$ satisfazendo as hipóteses (3.60), (3.61) e (3.75). Observamos que com os mesmos argumentos usados no Teorema 3.3.2 podemos obter (3.71). 
Como nesse caso $g$ não satisfaz a hipótese (3.62), o processo de passar o limite no segundo termo do lado esquerdo da igualdade em (3.66) é um pouco mais delicado. Neste ponto, o trabalho de Boccardo \& Gallouet cita o trabalho de Gallouet \& Morel, em [11], para podermos passar o limite em (3.66). Esse método consiste em mostrar que a sequência $\left(g_{n}\right)$ é equiintegrável e depois usar o Teorema de Vitali, para concluirmos que

$$
g_{n}\left(\cdot, u_{n}\right) \longrightarrow g(\cdot, u) \text { em } L_{l o c}^{1}(\Omega)
$$

Por hipótese, vamos considerar que $B=\|f\|_{L^{1}}$ e assumir que $f_{n}$ converge para $f$ em $L^{1}(\Omega)$. Como em (3.71), temos que:

$$
u_{n} \rightarrow u, \quad \text { q.t.p. }
$$

e sendo $g$ contínua no seu segundo argumento pela hipótese (3.60), segue que:

$$
g_{n}\left(\cdot, u_{n}\right) \rightarrow g(\cdot, u) \quad \text { q.t.p. }
$$

Para que $u$ satisfaça $(3.63)$, é suficiente provarmos que $g_{n}\left(\cdot, u_{n}\right)$ converge para $g(\cdot, u)$ em $L_{l o c}^{1}(\Omega)$ e $g(\cdot, u) \in L^{1}(\Omega)$. De fato, estabelecidas tais afirmações junto com (3.71) e pelo fato de $f_{n}$ convergir em $L^{1}$ para $f$, podemos passar o limite em (3.66) e obter (3.63).

Afirmação 3.3.3. $g_{n}\left(\cdot, u_{n}\right)$ converge para $g(\cdot, u)$ em $L_{l o c}^{1}(\Omega)$ e $g(\cdot, u) \in L^{1}(\Omega)$.

Pelo Teorema de Vitali, para mostrarmos tal afirmação, basta provarmos que:

$$
g_{n}\left(\cdot, u_{n}\right) \text { é equi-integrável em } K \text { para todo } K \subset \Omega, K \text { compacto. }
$$

Sejam $K \subset \Omega$, com $K$ compacto, $\varepsilon>0$ e $B \subset K$, onde $B$ é mensurável.

Note que como $\left(u_{n}\right)$ é limitado em $L^{1}(\Omega)$ (por (3.69) e pelo Lema 3.1.2), temos:

$$
\operatorname{med}\left(\left\{\left|u_{n}\right|>t\right\}\right) \rightarrow 0 \text {, uniformemente quando } t \rightarrow \infty \text {, para todo } n \in \mathbb{N} \text {. }
$$

Além disso, como $f_{n}$ é equi-integrável em $\Omega$, em particular em $K$, então existe $\delta_{1}>0$, tal que para todo $E \subset K$ com $\operatorname{med}(E)<\delta_{1}$, temos

$$
\int_{E}\left|f_{n}\right| d x \leq \varepsilon, \quad \text { para todo } n \in \mathbb{N} .
$$

Para $\delta_{1}>0$, existirá um $t_{0}$ tal que $\operatorname{med}\left(\left\{\left|u_{n}\right|>t_{0}\right\}\right)<\delta_{1}$ (por (3.78)), então:

$$
\int_{\left(\left\{\left|u_{n}\right|>t_{0}\right\}\right.}\left|f_{n}\right| d x \leq \varepsilon, \quad \text { para todo } n \in \mathbb{N} .
$$


Por (3.3.1), obtemos:

$$
\int_{\left(\left\{\left|u_{n}\right|>t_{0}\right\}\right.}\left|g_{n}\left(x, u_{n}\right)\right| d x \leq \varepsilon, \quad \text { para todo } n \in N .
$$

Note que $B=\left(B \cap\left\{\left|u_{n}\right|>t_{0}\right\}\right) \cup\left(B \cap\left\{\left|u_{n}\right| \leq t_{0}\right\}\right)$. Sendo assim, temos:

$$
\begin{aligned}
\int_{B}\left|g_{n}\left(x, u_{n}\right)\right| d x & =\int_{B \cap\left\{\left|u_{n}\right|>t_{0}\right\}}\left|g_{n}\left(x, u_{n}\right)\right| d x+\int_{B \cap\left\{\left|u_{n}\right| \leq t_{0}\right\}}\left|g_{n}\left(x, u_{n}\right)\right| d x \\
& \leq \varepsilon+\int_{B \cap\left\{\left|u_{n}\right| \leq t_{0}\right\}} \sup \left\{\left|g_{n}\left(x, u_{n}\right)\right|,\left|u_{n}\right| \leq t_{0}\right\} d x .
\end{aligned}
$$

Pela hipótese (3.75), $\sup \left\{\left|g_{n}\left(x, u_{n}\right)\right|,\left|u_{n}\right| \leq t_{0}\right\} \in L_{l o c}^{1}(\Omega)$, para todo $n \in \mathbb{N}$, então temos que existe $\delta_{2}>0$, tal que

$$
\int_{B \cap\left\{\left|u_{n}\right| \leq t_{0}\right\}} \sup \left\{\left|g_{n}\left(x, u_{n}\right)\right|,\left|u_{n}\right| \leq t_{0}\right\} d x \leq \varepsilon,
$$

sempre que $\operatorname{med}\left(B \cap\left\{\left|u_{n}\right| \leq t_{0}\right\}\right)<\delta_{2}$. Dessa forma, tomamos $B \subset K$ tal que $\operatorname{med}(B)<\delta_{2}$, e assim,

$$
\int_{B}\left|g_{n}\left(x, u_{n}\right)\right| d x \leq 2 \varepsilon .
$$

Logo, $g_{n}\left(\cdot, u_{n}\right)$ é equi-integrável em $K$ para todo $K \subset \Omega, K$ compacto. Portanto a Afirmação 3.3.1, está provada.

Afirmação 3.3.4. $g(\cdot, u) \in L^{1}(\Omega)$.

Pela hipótese de $f_{n}$ convergir em $L^{1}(\Omega)$ para $f$ junto com (3.76), aplicamos o Lema de Fatou e, assim, temos:

$$
\begin{aligned}
\int_{\Omega}|g(\cdot, u)|=\int_{\Omega} \liminf _{n \rightarrow \infty}\left|g_{n}\left(\cdot, u_{n}\right)\right| & \leq \liminf _{n \rightarrow \infty} \int_{\Omega}\left|g\left(\cdot, u_{n}\right)\right| \\
& \leq \liminf _{n \rightarrow \infty} \int_{\Omega}\left|f_{n}\right| \\
& =\int_{\Omega}|f| .
\end{aligned}
$$

Portanto, a Afirmação 3.3.2 está provada.

Com esses resultados, temos que $u$ satisfaz (3.74). 
Observamos que com a hipóteses sobre a $g$ no Teorema 3.3.3, podemos não ter existência de solução fraca quando $f \in M(\Omega)$. No trabalho de Brezis, em [3], foi mostrado que o problema:

$$
\left\{\begin{aligned}
-\Delta u+u^{3}=\delta, & \text { em } \Omega, \\
u=0, & \text { sobre } \partial \Omega
\end{aligned}\right.
$$

não tem solução fraca quando $N=3$ e $0 \in \Omega$. 


\section{Referências Bibliográficas}

[1] BARTLE, R. G.. The Elements of Integration, John Wiley \& Sons, Inc., 1966

[2] BOCCARDO, Lucio.; GALLOUET, Thierry.. Nonlinear elliptic and parabolic equations involving measure data, J. Funct. Anal. 87, 1989, no. 1, 149-169

[3] BREZIS, H.. Some variational problems of the Thomas-Fermi type, Variational inequalities and complementarity problems (Proc. Internat. School, Erice, 1978), pp. 53?73, Wiley, Chichester, 1980.

[4] BREZIS, H.. Functional Analysis, Sobolev Spaces and Partial Differential Equations, Springer, 2010.

[5] BROWDER, F.E.. Variational boundary value problems for quasi-linear elliptic equations of arbitrary order, Proc. Nat. Acad. Sci. U.S.A. 50, 1963, 31-37.

[6] BROWDER, F.E.. Nonlinear elliptic boundary value problems, Bull. Amer. Math. Soc. 69, 1963, 862-874.

[7] CIARLET, P.G.. Linear and Nonlinear Functional Analysis with Applications, Society for Insdustrial and Applied Mathematics, 2013.

[8] EVANS, Lawrence C.. Partial Differential Equations, American Mathematical Society, Vol. 19., 1997.

[9] FOLLAND, G.B.. Real Analysis: Modern Techniques and Their Applications, 2nd ed. A Whiley-Interscience Publication, 1999.

[10] FONSECA, Irene.; GANGBO, Wilfrid.. Degree Theory in Analysis and Aplications, Oxford Sciencee Publications, 1995. 
[11] GALlOUET, T.; MOREL, J.M.. On some semilinear problems in $L^{1}$,Boll. Un. Mat. Ital. A (6) 4, 1985, no. 1, 123-131.

[12] GALLOUET, T.. Équations elliptiques semilinéaires avec, pour la non linéarité, une condition de signe et une dépendance sous quadratique par rapport au gradient, Ann. Fac. Sci. Toulouse Math. (5) 9, 1988, no. 2, 161-169.

[13] LERAY, Jeans.; LIONS, J.L ..Quelques résulatats de Visik sur les problèmes elliptiques nonlinéaires par les méthodes de Minty-Browder, Bull. Soc. Math. France 93, 1965, 97107.

[14] GILBARG, David.; TRUDINGER, Neil, S.. Elliptic Partial Differential Equations of Second Order, Springer, 1998.

[15] MINTY, George J.. Monotone (nonlinear) operators in Hilbert space, Duke Math. J. 29, 1962, 341-346.

[16] MINTY, George J.. On the maximal domain of a "monotone" function, Michigan Math. J. 8, 1961, 135-137.

[17] MINTY, George J.. On a "monotonicity" method for the solution of non-linear equations in Banach spaces, Proc. Nat. Acad. Sci. U.S.A. 50, 1963, $1038 ? 1041$.

[18] MEDEIROS, L.A.; MIRANDA, M.M.. Espaços de Sobolev: Iniciação aos Problemas Elíticos não Homogêneos, UFRJ, 2000

[19] VISIK, M. I.. Boundary-value problems for quasilinear strongly elliptic systems of equations having divergence form, Dokl. Akad. Nauk SSSR 138, 1961, 518-521.

[20] VISIK, M. I.. Quasi-linear strongly elliptic systems of differential equations of divergence form, Trudy Moskov. Mat. Obsc. 12, 1963, 125-184. 